\title{
The Inverse Backscattering Problem in Three Dimensions
}

\author{
G. Eskin and J. Ralston, UCLA
}

Department of Mathematics, University of California, Los Angeles, CA 90024, USA

\begin{abstract}
This article is a study of the mapping from a potential $q(x)$ on $\mathbf{R}^{\mathbf{3}}$ to the backscattering amplitude associated with the Hamiltonian $-\Delta+q(x)$. The backscattering amplitude is the restriction of the scattering amplitude $a(\theta, \omega, k),(\theta, \omega, k) \in S^{2} \times S^{2} \times \mathbb{R}_{+}$, to $a(\theta,-\theta, k)$. We show that in suitable (complex) Banach spaces the map from $q(x)$ to $a(x /|x|,-x /|x|,|x|)$ is usually a local diffeomorphism. Hence in contrast to the overdetermined problem of recovering $q$ from the full scattering amplitude the inverse backscattering problem is well posed.
\end{abstract}

This article is a study of the mapping from a potential on $\mathbf{R}^{\mathbf{3}}$ to its quantum mechanical scattering amplitude. The scattering amplitude associated with a potential $q(x)$ can be described as follows. One assumes that for each $k>0$ and each $\omega \in S^{2}$,

$$
\left(-\Delta+q-k^{2}\right) u=0
$$

has a unique solution of the form $\exp (i k \omega \cdot x)+v(x, \omega, k)$ such that $v=\lim v_{\varepsilon}$, where $v_{\varepsilon}$ is the square-integrable solution of $\varepsilon \downarrow 0$

$$
-\Delta v_{\varepsilon}+q v_{\varepsilon}-(k+i \varepsilon)^{2} v_{\varepsilon}=-e^{i k \omega \cdot x} q .
$$

Much work has been devoted to showing that, under general hypotheses on $q, v(x, \omega, k)$ exists and is unique (see Agmon [1], and the references given there). When $q \in C_{0}^{\infty}\left(\mathbf{R}^{3}\right)$ and hence $\Delta v+k^{2} v \in C_{0}^{\infty}\left(\mathbf{R}^{3}\right)$, it is an elementary consequence of (I.1) that

$$
\begin{aligned}
& v(x)=-\frac{1}{4 \pi} \int_{\mathbf{R}^{3}} \frac{e^{i k|x-y|}}{|x-y|}\left(\Delta+k^{2}\right) v(y) d y, \text { and hence } \\
& v(x)=\left(\frac{e^{i k|x|}}{4 \pi|x|}\right)\left(a(x /|x|, \omega, k)+O\left(|x|^{-1}\right)\right)
\end{aligned}
$$

as $|x| \rightarrow \infty$. The function $a(\theta, \omega, k)$ on $S^{2} \times S^{2} \times \mathbf{R}_{+}$is known as the scattering amplitude. If we replace functions in (I.2) by their Fourier transforms, we have 


$$
\begin{aligned}
v(x, \omega, k) & =\lim _{\varepsilon \downarrow 0}(2 \pi)^{-3} \int_{\mathbf{R}^{3}} \frac{e^{i x \cdot \xi} g(\xi, \omega, k)}{|\xi|^{2}-(k+i \varepsilon)^{2}} d \xi \\
& \equiv(2 \pi)^{-3} \int_{\mathbf{R}^{3}} \frac{e^{i x \cdot \xi} g(\xi, \omega, k)}{|\xi|^{2}-(k+i 0)^{2}} d \xi,
\end{aligned}
$$

where $g$ is the Fourier transform of $-\left(\Delta+k^{2}\right) v$. Evaluating (I.3) in spherical coordinates and using stationary phase in the angular integration to derive asymptotics as $|x| \rightarrow \infty$, we find that

$$
a(\theta, \omega, k)=g(k \theta, \omega, k) .
$$

Given $q \in C_{0}^{\infty}\left(\mathbf{R}^{3}\right)$, taking the Fourier transform of (I.1) and the limit $\varepsilon \downarrow 0$, one arrives at

$$
g(\xi, \omega, k)+(2 \pi)^{-3} \int_{\mathbf{R}^{3}} \frac{\hat{q}(\xi-\eta) g(\eta, \omega, k)}{|\eta|^{2}-(k+i 0)^{2}} d \eta=-\hat{q}(\xi-k \omega) .
$$

In this article we will take (I.4) and (I.5) as the definition of the scattering amplitude, i.e., when the integral equation (I.5) has a unique solution $g$ for $(\omega, k) \in S^{2} \times \mathbf{R}_{+}$, the scattering amplitude is defined by (I.4).

Since we are dealing with a singular integral equation involving the Fourier transform of the potential $q$, we will assume $\hat{q}$ belongs to one of the weighted Hölder spaces $H_{\alpha, N}$ with $0<\alpha<1$ and $N>1$. Spaces of this type have been used in scattering theory by L. D. Faddeev in [3] and K. O. Friedrichs in [5]. The norm in $H_{\alpha, N}$ is $\|f\|_{\alpha, N}=\left\|\left(1+|\xi|^{2}\right)^{N / 2} f\right\|_{\alpha}$, where

$$
\|f\|_{\alpha}=\sup _{\substack{|\Delta| \leqq 1 \\ \xi \in \mathbf{R}^{3}}}\left(|f(\xi)|+|\Delta|^{-\alpha}|f(\xi+\Delta)-f(\xi)|\right),
$$

and $H_{\alpha, N}$ is defined as the closure of $C_{0}^{\infty}\left(\mathbf{R}^{3}\right)$ in this norm. We do not assume that $q$ is real-valued, though our main interest is in potentials with small imaginary parts.

As our title implies we are interested in the inverse problem of determining the potential given the scattering amplitude. This problem is quite overdetermined and there has been considerable work devoted to characterizing which scattering amplitudes actually arise for given classes of potentials, beginning with L. D. Faddeev [4] and more recently Newton [11], Beals-Coifman [2], NachmanAblowitz [9], Melin [7] and Novikov-Khenkin [6]. We are concerned here with the inverse backscattering problem, i.e. determining $q$ from $a(\omega,-\omega, k)$. In dimensions $n>1$ the only work that we know of is the numerical study of Bayliss, Lin and Morawetz [8] using wave equation methods, and the formal solution of the three-dimensional problem for small potentials by Prosser [13].

For technical reasons we will replace (I.5) by

$$
h(\xi, \zeta, k)+(2 \pi)^{-3} \int_{\mathbf{R}^{3}} \frac{\hat{q}(\xi-\eta) h(\eta, \zeta, k)}{|\eta|^{2}-(k+i 0)^{2}} d \eta=-\hat{q}(\xi-\zeta),
$$

where now $(\xi, \zeta, k)$ ranges over $\mathbf{R}^{3} \times \mathbf{R}^{3} \times \overline{\mathbf{R}}_{+}$, i.e. $k=0$ is now included. Thus (I.4) becomes

$$
a(\theta, \omega, k)=h(k \theta, k \omega, k)
$$


Let $H_{\alpha, N}^{r}$ denote the (real) subspace of $H_{\alpha, N}$ consisting of Fourier transforms of real-valued potentials, i.e. the set of $\hat{q} \in H_{\alpha, N}$ such that $\hat{q}(\xi)=\overline{\hat{q}(-\xi)}$. The backscattering map is well behaved on $H_{\alpha, N}$ and we have the following result which is proven in Corollary 3.5 and Remark 4 after Theorem 3.1 in the text:

Theorem A. The backscattering map

$$
S: \hat{q} \rightarrow h(\xi,-\xi,|\xi|)
$$

is a continuously Frechet differentiable function from an open, dense set $\mathcal{O}$ in $H_{\alpha, N}$ into $H_{\alpha, N}$. Moreover, $\mathcal{O} \cap H_{\alpha, N}^{r}$ is dense in $H_{\alpha, N}^{r}$.

Since continuously differentiable functions on complex Banach spaces are analytic, $S$ is analytic. The set $\mathcal{O}$ is the set of $\hat{q}$ such that $I+A(\hat{q}, k)$ is injective on $H_{\alpha, N}$ for $k \geqq 0$, where

$$
[A(\hat{q}, k) f](\xi)=(2 \pi)^{-3} \int_{\mathbf{R}^{3}} \frac{\hat{q}(\xi-\eta) f(\eta) d \eta}{|\eta|^{2}-(k+i 0)^{2}} .
$$

The proof that $h(\xi,-\xi,|\xi|)$ belongs to precisely the same space $H_{\alpha, N}$ as $\hat{q}(\xi)$ for $\hat{q} \in \mathcal{O}$, i.e. the proof of Theorem A, is quite technical and takes up about half of this paper.

Next we prove that the Frechet derivative of $S$ is a Fredholm operator of index zero for $\hat{q} \in \mathcal{O}$ (Theorem 4.3) and that $\mathcal{O} \cap H_{\alpha, N}^{r}$ is contained in a connected component $\mathcal{O}_{1}$ of $\mathcal{O}$ (Proposition 5.3). This leads to the following theorem (Theorem 5.4):

Theorem B. The Frechlet derivative of $S$ at $\hat{q}$ is an isomorphism of $H_{\alpha, N}$ for $\hat{q}$ in an open, dense subset $\mathcal{O}_{2}$ of $\mathcal{O}_{1}$. Moreover, $\mathcal{O}_{2} \cap H_{\alpha, N}^{r}$ is an open, dense subset of $H_{\alpha, N}^{r}$.

The implicit function theorem then implies:

Corollary C. $S$ is a local analytic homeomorphism in a neighbourhood of each $\hat{q} \in \mathcal{O}_{2}$.

This is the main result of this paper. Corollary C implies that (locally) recovering $\hat{q}$ from backscattering data is a well-posed problem, since small changes in $h(\xi,-\xi,|\xi|)$ will lead to small changes in $\hat{q}(\xi)$ in $H_{\alpha, N}$ norm. Note also that the results in Theorem $\mathrm{B}$ and Corollary $\mathrm{C}$ do not depend on the number of negative eigenvalues of $-\Delta+q$. This follows from the fact that $\mathcal{O}_{2}$ is a subset of the connected set $\mathcal{O}_{1}$.

Even the backscattering problem is overdetermined when we restrict the domain of our mapping to real-valued potentials. Therefore in the final section we consider a restricted backscattering problem for the case of real-valued potentials. Let $S_{r}$ denote the mapping

$$
S_{r}: \hat{q} \rightarrow \frac{h(\xi,-\xi,|\xi|)+\overline{h(-\xi, \xi,|\xi|)}}{2} .
$$

Note that $\mathscr{F}^{-1} S_{r}$ is the real part of $\mathscr{F}^{-1} S$. This map is well-behaved on $H_{\alpha, N}^{r}: S_{r}$ is real-analytic with a Frechet derivative which is Fredholm and index zero for $\hat{q} \in H_{\alpha, N}^{r} \cap \mathcal{O}$, (Theorem 6.1). However, we only know that its Frechet derivative is an isomorphism on an open dense set $\mathcal{O}_{2}^{r}$ of the component $\mathcal{O}_{1}^{r}$ of $H_{\alpha, N}^{r} \cap \mathcal{O}$ containing the zero potential (Theorem 6.2). The component $\mathcal{O}_{1}^{r}$ does contain all $\hat{q}$ such that $q \in C_{0}^{\infty}\left(\mathbf{R}^{3}\right)$ and $-\Delta+q$ has no bound states with energies $E \leqq 0$ or 
half-bound states at $E=0$ (Proposition 6.3). We plan to study other approaches to the formulation of the restricted backscattering problem in the future.

\section{Section 1. Preliminaries}

We will use the weight function $\Lambda(\xi)=\left(1+|\xi|^{2}\right)^{1 / 2}$ and the Lipschitz norms

$$
\|f\|_{\alpha}=\sup \left(|f(\xi)|+\frac{|f(\xi+\Delta)-f(\xi)|}{|\Delta|^{\alpha}}\right),
$$

where $0<\alpha \leqq 1$ and the supremum is taken over $\left\{\xi \in \mathbf{R}^{3}, \Delta \in \mathbf{R}^{\mathbf{3}}: 0<|\Delta| \leqq 1\right\}$. The Banach space of all functions $f$ on $\mathbf{R}^{3}$ with $\|f\|_{\alpha}<\infty$ will be denoted by $C^{\alpha}\left(\mathbf{R}^{3}\right)$. We also use $\|f\|_{0}$ to denote the supremum of $|f(\xi)|$ over $\mathbf{R}^{3}$. The principal Banach spaces in this paper are $H_{\alpha, N}, 0<\alpha<1, N>1$, the closures of $C_{0}^{\infty}\left(\mathbf{R}^{3}\right)$ in the norms

$$
\|f\|_{\alpha, N}=\left\|\Lambda^{N} f\right\|_{\alpha} .
$$

While $H_{\alpha, N}$ does not contain all functions $f$ on $\mathbf{R}^{3}$ with $\|f\|_{\alpha, N}<\infty$, one does have the following.

Lemma 1.1. $H_{\alpha, N}$ contains all functions $f$ on $\mathbf{R}^{3}$ such that $\|f\|_{\alpha^{\prime}, N^{\prime}}<\infty$ for some $\alpha^{\prime}>\alpha$ and $N^{\prime}>N$.

Proof. Let $j_{\varepsilon}$ be the standard mollifier and choose $\varphi \in C_{0}^{\infty}\left(\mathbf{R}^{3}\right)$ with $\varphi(\xi)=1$ for $|\xi|<1$. Then for $R \geqq 1$

$$
\|(1-\varphi(\cdot / R)) f\|_{\alpha, N} \leqq C R^{N-N^{\prime}}\|f\|_{\alpha, N^{\prime}}
$$

and for fixed $R$, setting $g(\xi)=\varphi(\xi / R) f(\xi)$,

$$
\begin{aligned}
& \left\|g-j_{\varepsilon} * g\right\|_{\alpha, N} \\
& \quad \leqq C\left(\sup |g(\xi+\eta)-g(\xi)|+\sup \frac{|g(\xi+\eta+\Delta)-g(\xi+\Delta)-g(\xi+\eta)+g(\xi)|}{|\Delta|^{\alpha}}\right),
\end{aligned}
$$

where the suprema are taken over $\{\xi, \eta, \Delta:|\eta| \leqq \varepsilon,|\Delta| \leqq 1\}$. Thus

$$
\left\|g-j_{\varepsilon} * g\right\|_{\alpha, N} \leqq C\left(\varepsilon^{\alpha^{\prime}}\|g\|_{\alpha^{\prime}}+\sup _{|\Delta| \leqq 1}\left(\frac{\varepsilon^{\alpha^{\prime}-\alpha}}{|\Delta|^{\alpha}}\|g(\cdot+\Delta)-g(\cdot)\|_{\alpha^{\prime}-\alpha}\right)\right) .
$$

Hence, since $|\Delta|^{-\alpha}\|g(\cdot+\Delta)-g(\cdot)\|_{\alpha^{\prime}-\alpha} \leqq 3\|g\|_{\alpha^{\prime}}$,

$$
\left\|g-j_{\varepsilon} * g\right\|_{\alpha, N} \leqq C \varepsilon^{\alpha^{\prime}-\alpha}\|g\|_{\alpha^{\prime}} .
$$

We will also deal with functions defined on $\mathbf{R}^{3} \times \mathbf{R}^{3} \times \overline{\mathbf{R}}_{+}$. For functions on $\overline{\mathbf{R}}_{+}$, we define

$$
\|f\|_{\alpha}=\sup \left(|f(k)|+\frac{|f(k+\Delta)-f(k)|}{\Delta^{\alpha}}\right),
$$

where the supremum is taken over $\left\{k \in \overline{\mathbf{R}}_{+}, 0<\Delta \leqq 1\right\}$. Note that, since we take the supremum in $k$ and $\Delta,\|f\|_{\alpha}<\infty$ does imply $f \in C^{\alpha}[0, \infty)$. For $0<\alpha<1$, we define a $C^{\alpha}$-norm on functions on $\mathbf{R}^{3} \times \mathbf{R}^{3} \times \overline{\mathbf{R}}_{+}$by 


$$
\|f\|_{\alpha}=\sup \left(\|f(\cdot, \zeta, k)\|_{\alpha}+\|f(\xi, \cdot, k)\|_{\alpha}+\|f(\xi, \zeta, \cdot)\|_{\alpha}\right)
$$

with the supremum taken over $(\xi, \zeta, k) \in \mathbf{R}^{3} \times \mathbf{R}^{3} \times \overline{\mathbf{R}}_{+}$.

Translations of functions will often be denoted by subscripts, i.e. $f_{\zeta}(\xi)=f(\xi-\zeta)$. In particular we will often use $\Lambda_{\zeta}(\xi)$ for $\Lambda(\xi-\zeta)$.

\section{Section 2. Estimates of the Operator $A(\hat{q}, k)$}

We define for $\hat{q} \in H_{\alpha, N}$ and $f \in C_{0}^{\infty}\left(\mathbf{R}^{3}\right)$,

$$
[A(\hat{q}, k) f](\xi)=(2 \pi)^{-3} \int_{\mathbf{R}^{3}} \frac{\hat{q}(\xi-\eta) f(\eta) d \eta}{|\eta|^{2}-(k+i 0)^{2}} .
$$

Theorem 2.1. The operator $A(\hat{q}, k)$ satisfies the following estimate for $\zeta \in \mathbf{R}^{3}$ and $k \geqq 0$,

$$
\left\|\Lambda_{\zeta}^{N} A(\hat{q}, k) \Lambda_{\zeta}^{-N} \Lambda^{\delta} f\right\|_{\alpha} \leqq \frac{C}{(1+k)^{\gamma}}\|\hat{q}\|_{\alpha, N}\|f\|_{\alpha-\varepsilon},
$$

where $0<\alpha<1, N>1,0 \leqq \varepsilon<\alpha, 0 \leqq \delta<\min \{1, N-1\}$, and $\gamma<\min \{1-\delta$, $N-1-\delta\}$. The constant $C$ is independent of $k, \zeta, \hat{q}$ and $f$.

Theorem 2.1 is the principal estimate in this article. To prove it we need to know the asymptotic behavior of integrals of the weight functions.

Lemma 2.2. Define for $k>0, N>0$ and $(\xi, \zeta) \in \mathbf{R}^{6}$,

Then

$$
I(k, \xi, \zeta)=\int_{|\omega|=1} \frac{\left(1+|\xi-\zeta|^{2}\right)^{N / 2}}{\left(1+|\xi-k \omega|^{2}\right)^{N / 2}\left(1+|k \omega-\zeta|^{2}\right)^{N / 2}} d \omega .
$$

$$
I(k, \xi, \zeta) \leqq C_{N} \max \left\{(1+k)^{-2} \log (1+k),(1+k)^{-N}\right\} .
$$

Proof of Lemma 2.2.

$$
\begin{aligned}
I(k) & \leqq C_{N} \int_{|\omega|=1}\left[\left(1+|\xi-k \omega|^{2}\right)^{-N / 2}+\left(1+|k \omega-\zeta|^{2}\right)^{-N / 2}\right] d \omega \\
& \leqq 2 C_{N} \sup _{\xi} \int_{|\omega|=1}\left(1+|\xi-k \omega|^{2}\right)^{-N / 2} d \omega .
\end{aligned}
$$

Introducing spherical coordinates with the $z$-axis in direction $\xi$,

$$
\begin{aligned}
\int_{|\omega|=1}\left(1+|\xi-k \omega|^{2}\right)^{-N / 2} d w & =2 \pi \int_{0}^{\pi}\left(1+|\xi|^{2}-2|\xi| k \cos \theta+k^{2}\right)^{-N / 2} \sin \theta d \theta \\
& =2 \pi \int_{-1}^{1}\left(1+|\xi|^{2}-2|\xi| k \tau+k^{2}\right)^{-N / 2} d \tau .
\end{aligned}
$$

Letting $u=|\xi|^{2}-2|\xi| k \tau+k^{2}$, we have

$$
\begin{aligned}
\int_{|\omega|=1}\left(1+|\xi-k \omega|^{2}\right)^{-N / 2} d \omega=\frac{\pi}{|\xi| k} \int_{(|\xi|-k)^{2}}^{(|\xi|+k)^{2}}(1+u)^{-N / 2} d u & \text { if } N=2 \\
= & \frac{\pi}{|\xi| k} \begin{cases}\ln \left(\frac{(|\xi|+k)^{2}+1}{(|\xi|-k)^{2}+1}\right) & \text { if } \quad N \neq 2 . \\
\frac{2}{N-2}\left(\left(1+(k-|\xi|)^{2}\right)^{(2-N) / 2}-\left(1+(k+|\xi|)^{2}\right)^{(2-N) / 2}\right)\end{cases}
\end{aligned}
$$


If ||$\xi|-k|>\frac{1}{2} k$, we have

$$
\int_{|\omega|=1}\left(1+|\xi-k \omega|^{2}\right)^{-N / 2} d \omega \leqq 4 \pi\left(1+\frac{1}{4} k^{2}\right)^{-N / 2}
$$

and, if ||$\xi|-k|<\frac{1}{2} k$, formula (2.1) shows

$$
\int_{|\omega|=1}\left(1+|\xi-k \omega|^{2}\right)^{-N / 2} d \omega \leqq \frac{2 \pi}{k^{2}} \begin{cases}\frac{2}{N-2} & \text { if } \quad N>2 \\ \ln \left(1+\frac{25 k^{2}}{4}\right) & \text { if } N=2 \\ \frac{2}{2-N}\left(1+\frac{25 k^{2}}{4}\right)^{(2-N) / 2} & \text { if } \quad N<2 .\end{cases}
$$

Thus we have the desired estimate (note that for $0 \leqq k \leqq 1$ the estimate is trivial).

An immediate corollary of Lemma 2.2 is the following.

Lemma 2.3. For $0<\delta<\min \{1, N-1\}$ let

$$
J(k, \xi, \zeta)=\int_{|| \eta|-k|>1} \frac{\left(1+|\xi-\zeta|^{2}\right)^{N / 2}\left(1+|\eta|^{2}\right)^{\delta / 2}}{\left(1+|\xi-\eta|^{2}\right)^{N / 2}\left(|\eta|^{2}-k^{2}\right)\left(1+|\eta-\zeta|^{2}\right)^{N / 2}} d \eta .
$$

Then for $N>1+\delta$ and $\gamma<\min \{N-1-\delta, 1-\delta\}$ we have $J(k, \xi, \zeta) \leqq C_{\gamma, N, \delta}(1+k)^{-\gamma}$.

Proof of Lemma 2.3. By applying Lemma 2.2 with $|\eta|$ playing the role of $k$ and $\omega=\eta /|\eta|$, we see

$$
J \leqq C \int_{|| \eta|-k| \geqq 1} \frac{\left(1+|\eta|^{2}\right)^{\delta / 2}|\eta|^{2} d|\eta|}{\left.|| \eta\right|^{2}-k^{2} \mid\left(1+|\eta|^{2}\right)^{\beta / 2}}
$$

where $\beta=2$ if $N>2$, and $\beta=N-\varepsilon, \varepsilon>0$, for $N \leqq 2$. Substituting $k \eta^{\prime}=\eta$, we have

$$
J \leqq C k \int_{|| \eta^{\prime}|-1|>k^{-1}} \frac{\left(1+k^{2}\left|\eta^{\prime}\right|^{2}\right)^{(\delta-\beta) / 2}\left|\eta^{\prime}\right|^{2} d\left|\eta^{\prime}\right|}{\left.|| \eta^{\prime}\right|^{2}-1 \mid} .
$$

For $k>1 / 2$ we have

$$
J \leqq C k k^{\delta-\beta} \int_{|| \eta^{\prime}|-1|>1 / k} \frac{\left|\eta^{\prime}\right|^{2+\delta-\beta}}{|| \eta^{\prime}|-1|\left(\left|\eta^{\prime}\right|+1\right)} d\left|\eta^{\prime}\right|
$$

and, hence for $\beta-\delta>1$, we have

$$
J \leqq C k^{1+\delta-\beta}(1+\ln k)
$$

For $k<1 / 2$, we have immediately from (2.2)

$$
J \leqq C \int_{1}^{\infty}\left(1+|\eta|^{2}\right)^{(\delta-\beta) / 2} d|\eta| .
$$

From (2.3) and (2.4) we conclude

$$
J \leqq C(1+k)^{-\gamma}
$$


for any $\gamma<\beta-\delta-1$ when $\beta-\delta>1$, which is the desired result for $N>2$. Choosing $\varepsilon$ so that $N-\varepsilon>1+\delta$, when $N \leqq 2$, completes the proof.

Proof of Theorem 2.1. We begin by reducing the theorem to the case $\varepsilon=0$. For this let $\Delta(\mu)$ denote the operator $(\Delta(\mu) f)(\xi)=f(\xi+\mu)-f(\xi)$. Then $\Delta(\mu) A(\hat{q}, k) f=$ $A(\Delta(\mu) \hat{q}, k) f$, and assuming Theorem 2.1 in the case $\varepsilon=0$, we have

$$
|\mu|^{-\varepsilon}\left\|\Lambda_{\zeta}^{N} \Delta(\mu) A(\hat{q}, k) \Lambda^{\delta} \Lambda_{\zeta}^{-N} f\right\|_{\alpha-\varepsilon} \leqq \frac{C}{(1+k)^{\gamma}}|\mu|^{-\varepsilon}\|\Delta(\mu) \hat{q}\|_{\alpha-\varepsilon, N}\|f\|_{\alpha-\varepsilon} .
$$

For $p \in \mathbf{R}$ the mean value theorem implies

$$
\left|\Delta(\mu) \Lambda^{p}(\xi)\right| \leqq|\mu|\left|\partial_{\xi} \Lambda^{p}\left(\xi^{\prime}\right)\right|
$$

where $\left|\xi^{\prime}-\xi\right|<|\mu|$. Since $\left|\partial_{\xi} \Lambda(\xi)\right| \leqq 1$, and hence $\Lambda\left(\xi^{\prime}\right) / \Lambda(\xi)$ and $\Lambda(\xi) / \Lambda\left(\xi^{\prime}\right)$ are bounded for $\left|\xi^{\prime}-\xi\right| \leqq 1$, we have for $|\mu| \leqq 1$,

$$
\left|\Delta(\mu) \Lambda_{\zeta}^{p}(\xi)\right| \leqq C|\mu| \Lambda_{\zeta}^{p-1}(\xi)
$$

As in the proof of Lemma 1.1, we have

$$
\sup _{|\mu| \leqq 1}|\mu|^{-\varepsilon}\|\Delta(\mu) \hat{q}\|_{\alpha-\varepsilon, N} \leqq 3\|\hat{q}\|_{\alpha, N} .
$$

Moreover, it is also true (see Proposition 8, Sect. 4, Chap. V in Stein [14]) that $\left(\|f\|_{\alpha^{\prime}}+\sup |\mu|^{-\alpha}\|\Delta(\mu) f\|_{\alpha^{\prime}}\right) \geqq 1 / C\|f\|_{\alpha+\alpha^{\prime}}$. Thus, using (2.5) we have,

$$
\begin{aligned}
\left\|\Lambda_{\zeta}^{N} A(\hat{q}, k) \Lambda^{\delta} \Lambda_{\zeta}^{-N} f\right\|_{\alpha} \leqq & C\left(\left\|\Lambda_{\zeta}^{N} A(\hat{q}, k) \Lambda^{\delta} \Lambda_{\zeta}^{-N} f\right\|_{\alpha-\varepsilon}\right. \\
& \left.+\sup _{|\mu| \leqq 1}|\mu|^{-\varepsilon}\left\|\Lambda_{\zeta}^{N} \Delta(\mu) A(\hat{q}, k) \Lambda^{\delta} \Lambda_{\zeta}^{-N} f\right\|_{\alpha-\varepsilon}\right) .
\end{aligned}
$$

Thus we only need to consider Theorem 2.1 in the case $\varepsilon=0$.

To prove Theorem 2.1 , we begin by defining $h(\xi, \eta)=\Lambda_{\xi}^{N}(\eta) \hat{q}(\xi-\eta) f(\eta)$. Then, using (2.5) we conclude

$$
\|h(\cdot, \eta)\|_{\alpha}+\|h(\xi, \cdot)\|_{\alpha} \leqq C\|\hat{q}\|_{\alpha, N}\|f\|_{\alpha}
$$

uniformly for $(\xi, \eta) \in \mathbf{R}^{6}$.

Next we decompose $(2 \pi)^{3} \Lambda_{\zeta}^{N} A(\hat{q}, k) \Lambda_{\zeta}^{-N} \Lambda^{\delta} f$ into three terms:

$$
\begin{aligned}
& \quad \int_{|| \eta|-k|>1} \frac{\Lambda_{\zeta}^{N}(\xi) \Lambda^{\delta}(\eta)}{\Lambda_{\zeta}^{N}(\eta) \Lambda_{\xi}^{N}(\eta)} \frac{h(\xi, \eta)}{|\eta|^{2}-k^{2}} d \eta \\
& \quad+\int_{|| \eta|-k|<1} \frac{\Lambda_{\zeta}^{N}(\xi)}{|\eta|^{2}-k^{2}}\left[\frac{\Lambda^{\delta}(\eta) h(\xi, \eta)}{\Lambda_{\zeta}^{N}(\eta) \Lambda_{\xi}^{N}(\eta)}-\frac{\Lambda^{\delta}(k \omega) h(\xi, k \omega)}{\Lambda_{\zeta}^{N}(k \omega) \Lambda_{\zeta}^{N}(k \omega)}\right] d \eta \\
& \quad+\int_{|| \eta|-k|<1} \frac{\Lambda_{\zeta}^{N}(\xi) \Lambda^{\delta}(k \omega) h(\xi, k \omega)}{\left(|\eta|^{2}-(k+i 0)^{2}\right) \Lambda_{\zeta}^{N}(k \omega) \Lambda_{\xi}^{N}(k \omega)} d \eta \\
& \equiv I_{1}+I_{2}+I_{3},
\end{aligned}
$$

where $\omega=\eta /|\eta|$ in $I_{2}$ and $I_{3}$. In $I_{3}$ we introduce polar coordinates and compute

$$
I_{3}=\int_{S^{2}} \frac{\Lambda_{\zeta}^{N}(\xi) h(\xi, k \omega)}{\Lambda_{\zeta}^{N}(k \omega) \Lambda_{\xi}^{N}(k \omega)} d \omega \int_{|| \eta|-k|<1,|\eta|>0} \frac{\left(1+k^{2}\right)^{\delta / 2}|\eta|^{2} d|\eta|}{|\eta|^{2}-(k+i 0)^{2}} .
$$




\section{Moreover}

$$
\begin{aligned}
& \lim _{\varepsilon \rightarrow 0} \int_{|| \eta|-k|<a,|\eta|>0} \frac{|\eta|^{2}}{|\eta|^{2}-(k+i \varepsilon)^{2}} d|\eta| \\
& \quad= \begin{cases}2 a-\frac{k}{2}\left(\ln \left(\frac{2 k+a}{2 k-a}\right)-\pi i\right) & \text { if } k>a \\
a+k-\frac{k}{2}\left(\ln \left(1+\frac{2 k}{a}\right)-\pi i\right) & \text { if } k \leqq a .\end{cases}
\end{aligned}
$$

Hence

$$
\int_{|| \eta|-k|<1,|\eta|>0} \frac{|\eta|^{2}}{|\eta|^{2}-(k+i 0)^{2}} d|\eta|=\left\{\begin{array}{lll}
\frac{k \pi i}{2}+O(1) & \text { as } & k \rightarrow \infty \\
1+O(k) & \text { as } \quad k \rightarrow 0 .
\end{array}\right.
$$

Applying Lemma 2.2 and (2.5) we have $\left|I_{3}\right| \leqq(1+k)^{\delta-\beta-1} \sup _{(\xi)}|h(\xi, \eta)|$ and for $|\mu| \leqq 1,|\mu|^{-\alpha}\left|\Delta(\mu) I_{3}\right| \leqq(1+k)^{\delta-\beta+1} \sup \|h(\cdot, \eta)\|_{\alpha}$, where as in the proof of Lemma $2.3 \beta=2$ for $N>2$ and $\beta=N-\varepsilon, \varepsilon>0$, for $N \leqq 2$. Taking $\varepsilon$ small enough that $N-1-\delta-\varepsilon>\gamma$, if $N \leqq 2$, this shows that $I_{3}$ satisfies the estimate of the theorem. Hence we need only consider $I_{1}$ and $I_{2}$.

The estimates of $I_{1}$ follow immediately from Lemma 2.3 and (2.5). We have $\left|I_{1}\right| \leqq C(1+k)^{-\gamma} \sup _{(\xi, \eta)}|h(\xi, \eta)|$ and for $|\mu| \leqq 1,|\mu|^{-\alpha}\left|\Delta(\mu) I_{1}\right| \leqq C(1+k)^{-\gamma} \sup _{\eta}\|h(\cdot, \eta)\|_{\alpha}$, which again is the estimate of the theorem.

The estimate of $\left|I_{2}\right|$ is also easy. Once again (2.5) implies for ||$\eta|-k|<1$,

$$
\left|\frac{\Lambda^{\delta}(\eta) h(\xi, \eta)}{\Lambda_{\zeta}(\eta) \Lambda_{\xi}^{N}(\eta)}-\frac{\Lambda^{\delta}\left(k \frac{\eta}{|\eta|}\right) h\left(\xi, k \frac{\eta}{|\eta|}\right)}{\Lambda_{\zeta}^{N}\left(k \frac{\eta}{|\eta|}\right) \Lambda_{\xi}^{N}\left(k \frac{\eta}{|\eta|}\right)}\right| \leqq C|| \eta|-k|^{\alpha} \frac{\Lambda^{\delta}(\eta)}{\Lambda_{\zeta}^{N}(\eta) \Lambda_{\xi}^{N}(\eta)} \sup _{\xi}\|h(\xi, \cdot)\|_{\alpha} .
$$

Hence by Lemma 2.2

$$
\left|I_{2}\right| \leqq C \int_{|| \eta|-k|<1} \frac{\left(1+|\eta|^{2}\right)^{(\delta-\beta) / 2}|\eta|^{2}}{(|\eta|+k)|| \eta|-k|^{1-\alpha}} d|\eta|\left(\sup _{\xi}\|h(\xi, \eta)\|_{\alpha}\right),
$$

where $\beta$ is as before. This gives

$$
\left|I_{2}\right| \leqq C(1+k)^{\delta-\beta+1}\|\hat{q}\|_{\alpha, N}\|f\|_{\alpha}
$$

as desired.

It is the estimate of $\left|\Delta(\mu) I_{2}\right|$ that presents some problems. For this we need first to split the domain of integration in the integral into $\{|| \eta|-k|<2|\mu|\}$, getting $J_{1}$, and $\{2|\mu|<|| \eta|-k|<1\}$, getting $J_{2}$. To estimate $\Delta(\mu) J_{1}=J_{1}(\xi+\mu)-J_{1}(\xi)$, we use $\left|\Delta(\mu) J_{1}\right| \leqq\left|J_{1}(\xi+\mu)\right|+\left|J_{1}(\xi)\right|$. Since the procedure used to estimate $\left|I_{2}\right|$, shows that for $|\mu| \leqq 1$,

$$
\left|J_{1}(\xi+\mu)\right|+\left|J_{1}(\xi)\right| \leqq C \int_{|| \eta|-k|<2|\mu|}|| \eta|-k|^{-1+\alpha} d|\eta|(1+k)^{\delta-\beta+1}\|\hat{q}\|_{\alpha, N}\|f\|_{\alpha},
$$


and

$$
\int_{|| \eta|-k|<2|\mu|}|| \eta|-k|^{-1+\alpha} d|\eta| \leqq C|\mu|^{\alpha}
$$

we have the estimate required for $\left|\Delta(\mu) J_{1}\right|$.

To estimate $\Delta(\mu) J_{2}$ we must use the special form of $h(\xi, \eta)$, i.e. $h(\xi, \eta)=$ $\Lambda_{\xi}^{N}(\eta) \hat{q}(\xi-\eta) f(\eta)$. We have

$$
\begin{aligned}
\Delta(\mu) J_{2} & =\int_{2|\mu|<|| \eta|-k|<1} \frac{\left(\Delta(\mu) \Lambda_{\zeta}^{N}(\xi)\right)}{|\eta|^{2}-k^{2}}\left[\frac{\Lambda^{\delta}(\eta) h(\xi+\mu, \eta)}{\Lambda_{\zeta}^{N}(\eta) \Lambda_{\xi+\mu}^{N}(\eta)}-\frac{\Lambda^{\delta}(k \omega) h(\xi+\mu, k \omega)}{\Lambda_{\zeta}^{N}(k \omega) \Lambda_{\xi+\mu}^{N}(k \omega)}\right] d \eta \\
& +\int_{2|\mu|<|| \eta|-k|<1} \frac{\Lambda_{\zeta}^{N}(\xi)}{|\eta|^{2}-k^{2}}\left[\frac{\Lambda^{\delta}(\eta)}{\Lambda_{\zeta}^{N}(\eta)} \hat{q}(\xi+\mu-\eta)-\frac{\Lambda^{\delta}(k \omega)}{\Lambda_{\zeta}^{N}(k \omega)} \hat{q}(\xi+\mu-k \omega)\right] f(\eta) d \eta \\
& -\int_{2|\mu|<|| \mu|-k|<1} \frac{\Lambda_{\zeta}^{N}(\xi)}{|\eta|^{2}-k^{2}}\left[\frac{\Lambda^{\delta}(\eta)}{\Lambda_{\zeta}^{N}(\eta)} \hat{q}(\xi-\eta)-\frac{\Lambda^{\delta}(k \omega)}{\Lambda_{\zeta}^{N}(k \omega)} \hat{q}(\xi-k \omega)\right] f(\eta) d \eta \\
& +\int_{2|\mu|<|| \eta|-k|<1} \frac{\Lambda_{\zeta}^{N}(\xi)}{|\eta|^{2}-k^{2}} \frac{\Lambda^{\delta}(k \omega)}{\Lambda_{\zeta}^{N}(k \omega)}(\hat{q}(\xi+\mu-k \omega)-\hat{q}(\xi-k \omega))(f(\eta)-f(k \omega)) d \eta \\
& \equiv K_{1}+K_{2}-K_{3}+K_{4} .
\end{aligned}
$$

We can estimate $\left|K_{1}\right|$ exactly as $\left|I_{2}\right|$ was estimated and, using Lemma 2.2 and (2.5), one can easily verify that for $|\mu| \leqq 1$

$$
\left|K_{4}\right| \leqq C|\mu|^{\alpha}(1+k)^{\delta-\beta+1}\|\hat{q}\|_{\alpha, N}\|f\|_{\alpha} \int_{|| \eta|-k|<1}|| \eta|-k|^{-1+\alpha} d|\eta| .
$$

Hence $K_{4}$ also satisfies the required estimate.

In estimating $K_{2}-K_{3}$ we need to make the cancellation between $\hat{q}(\xi+\mu-\eta)$ and $\hat{q}(\xi-\eta)$ as good as possible. For this we replace $\eta$ in $K_{2}$ by $\eta+\mu$. This gives

$$
\begin{aligned}
K_{2}-K_{3}= & \int\left(\chi_{+}-\chi_{-}\right) \frac{\Lambda_{\zeta}^{N}(\xi)}{|\eta+\mu|^{2}-k^{2}}\left[\frac{\Lambda^{\delta}(\eta+\mu)}{\Lambda_{\zeta}^{N}(\eta+\mu)} \hat{q}(\xi+\mu-\eta-\mu)\right. \\
& \left.-\frac{\Lambda^{\delta}(k \tilde{\omega})}{\Lambda_{\zeta}^{N}(k \tilde{\omega})} \hat{q}(\xi+\mu-k \tilde{\omega})\right] f(\eta+\mu) d \eta+\int_{2|\mu|<|| \eta|-k|<1} \frac{\Lambda_{\zeta}^{N}(\xi)}{|\eta+\mu|^{2}-k^{2}} \\
& \cdot\left[\frac{\Lambda^{\delta}(\eta+\mu)}{\Lambda_{\zeta}^{N}(\eta+\mu)} \hat{q}(\xi-\eta)-\frac{\Lambda^{\delta}(k \tilde{\omega})}{\Lambda_{\zeta}^{N}(k \tilde{\omega})} \tilde{q}(\xi+\mu-k \tilde{\omega})\right](f(\eta+\mu)-f(\eta)) d \eta \\
& +\int_{2|\mu|<|| \eta|-k|<1} \Lambda_{\zeta}^{N}(\xi)\left[\frac{1}{|\eta+\mu|^{2}-k^{2}}-\frac{1}{|\eta|^{2}-k^{2}}\right] \\
& \cdot\left[\frac{\Lambda^{\delta}(\eta+\mu)}{\Lambda_{\zeta}^{N}(\eta+\mu)} \hat{q}(\xi+\mu-\eta-\mu)-\frac{\Lambda^{\delta}(k \tilde{\omega})}{\Lambda_{\zeta}^{N}(k \tilde{\omega})} \hat{q}(\xi+\mu-k \tilde{\omega})\right] f(\eta) d \eta \\
& +\int_{2|\mu|<|| \eta|-k|<1} \frac{\Lambda_{\zeta}^{N}(\xi)}{|\eta|^{2}-k^{2}}\left[\frac{\Lambda^{\delta}(\eta+\mu)}{\Lambda_{\zeta}^{N}(\eta+\mu)}-\frac{\Lambda^{\delta}(\eta)}{\Lambda_{\zeta}^{N}(\eta)}\right] \hat{q}(\xi-\eta) f(\eta) d \eta \\
& +\int_{2|\mu|<|| \eta|-k|<1} \frac{\Lambda_{\zeta}^{N}(\xi)}{\left(|\eta|^{2}-k^{2}\right)}\left(1+k^{2}\right)^{\delta / 2}
\end{aligned}
$$




$$
\begin{aligned}
& \cdot\left[\frac{\hat{q}(\xi-k \omega)}{\Lambda_{\zeta}^{N}(k \omega)}-\frac{\hat{q}(\xi+\mu-k \tilde{\omega})}{\Lambda_{\zeta}^{N}(k \tilde{\omega})}\right] f(\eta) d \eta \\
\equiv & L_{1}+L_{2}+L_{3}+L_{4}+L_{5} .
\end{aligned}
$$

Here $\tilde{\omega}=(\eta+\mu) /|\eta+\mu|$ and $\chi_{+}$is the characteristic function of $\{\eta: 2|\mu|<$ ||$\eta+\mu|-k|<1$ and ||$\eta|-k|>1$ or ||$\eta|-k|<2|\mu|\}$ and $\chi_{-}$is the characteristic function of $\{\eta: 2|\mu|<|| \eta|-k|<1$ and ||$\eta+\mu|-k|>1$ or ||$\eta+\mu|-k|<2|\mu|\}$.

The first two terms in the expansion of $K_{2}-K_{3}$ are like terms we have already considered. The integral $L_{1}$ can be estimated as $J_{1}$ was, and $L_{2}$ is another term like $K_{4}$. The remaining three terms require further explanation. Since

$$
|| \eta+\mu| \pm k|>\frac{1}{2}|| \eta| \pm k| \text { when }|| \eta|-k|>2|\mu|
$$

we have

$$
\begin{aligned}
\left|L_{3}\right| & \leqq C \int_{2|\mu|<|| \eta|-k|<1} \frac{|\mu|(|\eta|+1)|\eta|^{2}}{(|\eta|+k)^{2}}|| \eta|-k|^{\alpha-2} d|\eta|(1+k)^{\delta-\beta}\|\hat{q}\|_{\alpha, N}\|f\|_{0} \\
& \leqq C(1+k)^{\delta-\beta+1}\|\hat{q}\|_{\alpha, N}\|f\|_{0} \int_{2|\mu|<|| \eta|-k|<1} \frac{|\mu| d|\eta|}{\| \eta|-k|^{2-\alpha}} \\
& \leqq C(1+k)^{\delta-\beta+1}|\mu|^{\alpha}\|\hat{q}\|_{\alpha, N}\|f\|_{0} .
\end{aligned}
$$

By (2.5) we have

$$
\left|\frac{\Lambda^{\delta}(\eta+\mu)}{\Lambda_{\zeta}^{N}(\eta+\mu)}-\frac{\Lambda^{\delta}(\eta)}{\Lambda_{\zeta}^{N}(\eta)}\right| \leqq C|\mu| \frac{\Lambda^{\delta}(\eta)}{\Lambda_{\zeta}^{N}(\eta)} .
$$

Thus, we can estimate $L_{4}$ by

$$
\begin{aligned}
\left|L_{4}\right| & \leqq C|\mu|(1+k)^{\delta-\beta}\|\hat{q}\|_{0, N}\|f\|_{0} \int_{2|\mu|<|| \eta|-k|<1} \frac{|\eta|^{2}}{\left.|| \eta\right|^{2}-k^{2} \mid} d|\eta| \\
& \leqq C|\mu|(|| \ln |\mu| \mid+1)(1+k)^{\delta-\beta+1}\|\hat{q}\|_{0, N}\|f\|_{0} .
\end{aligned}
$$

Since $\alpha<1$, this is stronger than the estimate we need.

The term $L_{5}$ must be decomposed again (but this is the last decomposition we will use):

$$
\begin{aligned}
L_{5}= & \left(1+k^{2}\right)^{\delta / 2} \Lambda_{\zeta}^{N}(\xi)\left[\int_{2|\mu|<|| \eta|-k|<1}\left[\frac{\hat{q}(\xi-k \omega)}{\Lambda_{\zeta}^{N}(k \omega)}-\frac{\hat{q}(\xi+\mu-k \tilde{\omega})}{\Lambda_{\zeta}^{N}(k \tilde{\omega})}\right]\right] \\
& \cdot\left(\frac{f(\eta)-f(k \omega)}{|\eta|^{2}-k^{2}}\right) d \eta+\int_{2|\mu|<|| \eta|-k|<1}\left[\frac{\hat{q}(\xi-k \omega)}{\Lambda_{\zeta}^{N}(k \omega)}-\frac{\hat{q}(\xi+\mu-k \beta)}{\Lambda_{\zeta}^{N}(k \beta)}\right] \frac{f(k \omega)}{|\eta|^{2}-k^{2}} d \eta \\
& +\int_{2|\mu|<|| \eta|-k|<1}\left[\frac{\hat{q}(\xi+\mu-k \beta)}{\Lambda_{\zeta}^{N}(k \beta)}-\frac{\hat{q}(\xi+\mu-k \tilde{\omega})}{\Lambda_{\zeta}^{N}(k \tilde{\omega})}\right] \frac{f(k \omega)}{|\eta|^{2}-k^{2}} d \eta \\
\equiv & M_{1}+M_{2}+M_{3} .
\end{aligned}
$$

Here

$$
\beta=\frac{k \omega+\mu}{|k \omega+\mu|}=\left.\tilde{\omega}\right|_{|\eta|=k}
$$


The point of this decomposition is that the mean of the integrand in $M_{2}$ over spheres $|\eta|=c$ is independent of $|\eta|$, and hence we can estimate the integral in $|\eta|$ accurately. On the other hand $\tilde{\omega}-\beta$ is so small that we can control $M_{3}$.

We claim that

$$
|k(\tilde{\omega}-\beta)| \leqq C|\mu||| \eta|-k|(|\eta|+|\mu|)^{-1}+C|\mu|^{2}\left((k+|\mu|)^{-1}+(|\eta|+|\mu|)^{-1}\right)
$$

for all $\eta, k$ and $\mu$. One can arrive at this estimate in the following way. If $k<2|\mu|$, we have

$$
|k(\tilde{\omega}-\beta)| \leqq 2 k \leqq 4|\mu| \leqq \frac{12|\mu|^{2}}{k+|\mu|} .
$$

Similarly, if $|\eta|<2|\mu|$, we have

$$
|k(\tilde{\omega}-\beta)| \leqq 2 k \leqq 2|\eta|+2|| \eta|-k| \leqq \frac{12|\mu|^{2}}{|\eta|+|\mu|}+\frac{6|\mu||| \eta|-k|}{|\eta|+|\mu|} .
$$

When $|\eta|>2|\mu|$ and $k>2|\mu|$, we use Taylor series in $\mu$. Thus

$$
\begin{aligned}
\tilde{\omega} & =\frac{\eta+\mu}{|\eta+\mu|}=\frac{\eta+\mu}{|\eta|}\left(1+\frac{2 \mu \cdot \eta}{|\eta|^{2}}+\frac{|\mu|^{2}}{|\eta|^{2}}\right)^{-1 / 2} \\
& =\omega+\frac{\mu}{|\eta|}-\frac{(\mu \cdot \omega) \omega}{|\eta|}+O\left(\frac{|\mu|^{2}}{|\eta|^{2}}\right),
\end{aligned}
$$

and, since $\beta=\left.\tilde{\omega}\right|_{|\eta|=k}$,

$$
\beta=\omega+\frac{\mu}{k}-\frac{(\mu \cdot \omega) \omega}{k}+O\left(\frac{|\mu|^{2}}{k^{2}}\right)
$$

Thus

$$
\tilde{\omega}-\beta=\mu\left(\frac{k-|\eta|}{k|\eta|}\right)+(\mu \cdot \omega) \omega\left(\frac{|\eta|-k}{k|\eta|}\right)+O\left(\frac{|\mu|^{2}}{k^{2}}+\frac{|\mu|^{2}}{|\eta|^{2}}\right)
$$

and,

$$
|k(\tilde{\omega}-\beta)| \leqq \frac{2|\mu||k-| \eta||}{|\eta|}+C|\mu|^{2}\left(\frac{1}{k}+\frac{1}{|\eta|}+\frac{|| \eta|-k|}{|\eta|^{2}}\right) .
$$

Thus, since $2|\mu|<k$ and $2|\mu|<|\eta|$, wee see that (2.7) holds. However, since $|\mu|^{2}(k+|\mu|)^{-1} \leqq|\mu|^{2}(|\eta|+|\mu|)^{-1}+|\mu||| \eta|-k|(|\eta|+|\mu|)^{-1}$, we actually have

$$
|k(\beta-\tilde{\omega})| \leqq C \frac{\left.|\mu||| \eta|-k||+| \mu\right|^{2}}{|\eta|+|\mu|} .
$$

From (2.8) we have for $|\eta|>2|\mu|$

$$
\tilde{\omega}-\omega=\frac{\mu}{|\eta|}-\frac{(\mu \cdot \omega) \omega}{|\eta|}+O\left(\frac{|\mu|^{2}}{|\eta|^{2}}\right) .
$$

Thus for $|\eta|>2|\mu|$,

$$
|k(\tilde{\omega}-\omega)| \leqq C k \frac{|\mu|}{|\eta|+|\mu|},
$$


and this estimate also holds (with $C=6$ ) for $|\eta|<2|\mu|$. Finally, from (2.9) we see for $k>2|\mu|$,

$$
|k(\omega-\beta)| \leqq 2|\mu|+C \frac{|\mu|^{2}}{k} \leqq C|\mu|,
$$

and again this estimate also holds (with $C=4$ ) for $k<2|\mu|$. We will use (2.11) to estimate $M_{1},(2.12)$ to estimate $M_{2}$ and (2.10) to estimate $M_{3}$. We have by Lemma 2.2 and (2.11),

$$
\begin{aligned}
\left|M_{1}\right| & \leqq C(1+k)^{\delta-\beta}|\mu|^{\alpha}\|\hat{q}\|_{\alpha, N}\|f\|_{\alpha} \int_{|| \eta|-k|<1}\left(1+\frac{k^{\alpha}}{(|\eta|+|\mu|)^{\alpha}}\right) \frac{|\eta|^{2} d|\eta|}{(|\eta|+k)|| \eta|-k|^{1-\alpha}} \\
& \leqq C(1+k)^{\delta-\beta+1}|\mu|^{\alpha}\|\hat{q}\|_{\alpha, N} \| f_{\alpha}
\end{aligned}
$$

as desired.

The integral $M_{2}$ is given by

$$
M_{2}=\left(1+k^{2}\right)^{\delta / 2} P(k, \mu, \xi, \zeta) \int_{2|\mu|<|| \eta|-k|<1} \frac{|\eta|^{2}}{|\eta|^{2}-k^{2}} d|\eta|,
$$

where

$$
P=\Lambda_{\zeta}^{N}(\xi) \int_{S^{2}}\left(\frac{\hat{q}(\xi-k \omega)}{\Lambda_{\zeta}^{N}(k \omega)}-\frac{\hat{q}(\xi+\mu-k \beta)}{\Lambda_{\zeta}^{N}(k \beta)}\right) f(k \omega) d \omega .
$$

Lemma 2.2 and (2.12) show

We have

$$
|P| \leqq C(1+k)^{-\beta}|\mu|^{\alpha}\|\hat{q}\|_{\alpha, N}\|f\|_{0} .
$$

$\int_{1>|| \eta|-k|>2|\mu|} \frac{|\eta|^{2}}{|\eta|^{2}-k^{2}} d|\eta|=\frac{k}{2} \int_{1>|| \eta|-k|>2|\mu|} \frac{d|\eta|}{|\eta|-k}+\int_{1>|| \eta|-k|>2|\mu|} \frac{2|\eta|+k}{2(|\eta|+k)} d|\eta|$.

The second integral is bounded by 2 , and

$$
\int_{1>|| \eta \mid} \frac{d|\eta|}{|\eta|>2|\mu|}= \begin{cases}0, & k>1, \\ -\ln k, & 2|\mu|<k<1 \\ -\ln 2|\mu|, & k<2|\mu| .\end{cases}
$$

Since $k \ln k$ is bounded for $k<1$, we conclude from (2.13),

$$
\left|M_{2}\right| \leqq C(1+k)^{\delta-\beta}|\mu|^{\alpha}\|\hat{q}\|_{\alpha, N}\|f\|_{0},
$$

which suffices.

By Lemma 2.2 and (2.10) we have

$$
\begin{aligned}
\left|M_{3}\right| \leqq C(1+k)^{\delta-\beta}\|\hat{q}\|_{\alpha, N}\|f\|_{0} \\
\quad \int_{2|\mu|<|| \eta|-k|<1} \frac{|\eta|^{2}}{\|\left.\eta\right|^{2}-k^{2} \mid}\left(\frac{|\mu|^{\alpha}|| \eta|-k|^{\alpha}+|\mu|^{2 \alpha}}{(|\eta|+|\mu|)^{\alpha}}\right) d|\eta| \\
\leqq C(1+k)^{\delta-\beta+1-\alpha}\|\hat{q}\|_{\alpha, N}\|f\|_{0}\left(|\mu|^{\alpha}+|\mu|^{2 \alpha}(1+|\ln | \mu||)\right)
\end{aligned}
$$

which suffices. 
In addition to the estimate in Theorem 2.1 we also need control of Lipschitz norms in the variable $k$. This is provided by the following theorem.

Theorem 2.2. Let $\Delta(s), 0<s<1$ denote operator $(\Delta(s) f)(k)=f(k+s)-f(k)$. Then one has the estimate

$$
\begin{gathered}
\sup _{\zeta, \zeta} \Lambda_{\zeta}^{N}(\xi)\left|\int_{\mathbf{R}^{3}} f(\eta, \xi, \zeta, k)\left(\Delta(s)\left(\frac{1}{|\eta|^{2}-(k+i 0)^{2}}\right)\right) d \eta\right| \\
\leqq \frac{C s^{\alpha}}{(1+k)^{\gamma}} \sup _{\zeta, \zeta}\left\|\Lambda^{-\delta}(\cdot) \Lambda_{\xi}^{N}(\cdot) \Lambda_{\zeta}^{N}(\cdot) f(\cdot, \xi, \zeta, k)\right\|_{\alpha}
\end{gathered}
$$

with $C$ independent of $k$ for $\alpha, N, \delta$ and $\gamma$ in the set given in Theorem 2.1.

Proof of Theorem 2.2. Here we will write

$$
\begin{aligned}
\Lambda_{\zeta}^{N}(\xi) & \int_{\mathbf{R}^{3}} f(\eta)\left(\Delta(s)\left(\frac{1}{|\eta|^{2}-(k+i 0)^{2}}\right)\right) d \eta \\
= & \Lambda_{\zeta}^{N}(\xi) \int_{|| \eta|-k|>1} f(\eta)\left(\Delta(s)\left(\frac{1}{|\eta|^{2}-k^{2}}\right)\right) d \eta \\
& +\Lambda_{\zeta}^{N}(\xi) \int_{|| \eta|-k|<1} \Delta(s)\left((f(\eta)-f(k \omega))\left(\frac{1}{|\eta|^{2}-k^{2}}\right)\right) d \eta \\
& +\Lambda_{\zeta}^{N}(\xi) \int_{|| \eta|-k|<1} \Delta(s)\left(f(k \omega)\left(\frac{1}{|\eta|^{2}-(k+i 0)^{2}}\right)\right) d \eta \\
\equiv & I_{1}+I_{2}+I_{3},
\end{aligned}
$$

where $f(\eta)=f(\eta, \xi, \zeta, k)$ and $\Delta(s) f(k \omega)=f((k+s) \omega, \xi, \zeta, k)-f(k \omega, \xi, \zeta, k)$.

By Lemma 2.2 for some $\beta>1+\delta$, setting $h=\Lambda^{-\delta}(\eta) \Lambda_{\zeta}^{N}(\eta) \Lambda_{\xi}^{N}(\eta) f(\eta, \xi, \zeta, k)$, we have

$$
\begin{aligned}
\left|I_{1}\right| \leqq & C \sup _{\xi, \zeta} h \mid \int_{|| \eta|-k|>1} \frac{\left(k s+s^{2}\right)(1+|\eta|)^{-\beta+\delta}}{\left.|| \eta\right|^{2}-k^{2}|||\eta|^{2}-(k+s)^{2} \mid} d \eta \\
\leqq & C \sup _{\xi, \zeta}|h|(k+1) s \int_{|| \eta|-k|>1 / 2} \frac{(1+|\eta|)^{-\beta+\delta}}{|| \eta|-k|^{2}} d|\eta| \\
\leqq & C \sup _{\xi, \zeta}|h|(k+1) s\left[\int_{1 / 2<|| \eta|-k|<k / 2} \frac{(1+|\eta|)^{-\beta+\delta}}{|| \eta|-k|^{2}} d|\eta|\right. \\
& \left.+\int_{|| \eta|-k|>\max \{1 / 2, k / 2\}} \frac{(1+|\eta|)^{-\beta+\delta}}{|| \eta|-k|^{2}} d|\eta|\right] \leqq C \sup _{\xi, \zeta}|h|(k+1) s \\
& \cdot\left((1+k)^{-\beta+\delta} \int_{|| \eta|-k|>1 / 2}|| \eta|-k|^{-2} d|\eta|+(1+k)^{-2} \int_{|\eta|>0}(1+|\eta|)^{-\beta+\delta} d|\eta|\right) \\
\leqq & C\left(\sup _{\xi, \zeta}|h|\right) s(1+k)^{-\beta+\delta+1} .
\end{aligned}
$$


The last term $I_{3}$ is also easy to estimate,

$$
I_{3}=\Lambda_{\zeta}^{N}(\xi)\left((\Delta(s) g) \int_{S^{2}} f((k+s) \omega) d \omega+g \int \Delta(s)(f(k \omega)) d \omega\right)
$$

where (see (2.6))

$$
g(k)= \begin{cases}2-\frac{k}{2}\left(\ln \left(\frac{2 k+1}{2 k-1}\right)-\pi i\right) & \text { if } \quad k>1 \\ 1+k-\frac{k}{2}(\ln (2 k+1)-\pi i) & \text { if } \quad k<1\end{cases}
$$

Since $g$ has Lipschitz constant bounded on $\mathbf{R}_{+}$,

$$
\left|I_{3}\right| \leqq \frac{C s^{\alpha}}{(1+k)^{\beta-\delta-1}} \sup _{\xi, \zeta}\left\|\Lambda^{-\delta}(\cdot) \Lambda_{\xi}^{N}(\cdot) \Lambda_{\zeta}^{N}(\cdot) f(\cdot, \xi, \zeta, k)\right\|_{\alpha} .
$$

The term $I_{2}$ here we decompose to

$$
\begin{aligned}
I_{2}= & \Lambda_{\zeta}^{N}(\xi) \int_{|| \eta|-k|<2 s}(f(\eta)-f(k+s) \omega) \frac{d \eta}{|\eta|^{2}-(k+s)^{2}} \\
& -\Lambda_{\zeta}^{N}(\xi) \int_{|| \eta|-k|<2 s}(f(\eta)-f(k \omega)) \frac{d \eta}{|\eta|^{2}-k^{2}} \\
& +\Lambda_{\zeta}^{N}(\xi) \int_{2 s<|| \eta|-k|<1}(f(\eta)-f(k \omega))\left(\frac{1}{|\eta|^{2}-(k+s)^{2}}-\frac{1}{|\eta|^{2}-k^{2}}\right) d \eta \\
& +\Lambda_{\zeta}^{N}(\xi) \int_{2 s<|| \eta|-k|<1}(f(k \omega)-f((k+s) \omega))\left(\frac{1}{|\eta|^{2}-(k+s)^{2}}\right) d \eta \\
\equiv & J_{1}+J_{2}+J_{3}+J_{4} .
\end{aligned}
$$

Here $\left|J_{1}\right|$ and $\left|J_{2}\right|$ can be estimated in the same way that $\left|\Delta(\mu) J_{1}\right|$ was estimated in the proof of Theorem 2.1 with $s$ in place of $|\mu|$. Likewise $\left|J_{3}\right|$ can be estimated as $L_{3}$ was estimated. Finally $J_{4}$ is like $M_{2}$ in the proof of Theorem 2.1. Carrying out the integration in $|\eta|$, we have

$$
J_{4}=\left(\Lambda_{\zeta}^{N}(\xi) \int_{S^{2}}(f(k \omega)-f((k+s) \omega)) d \omega\right) P(k, s),
$$

where

$$
\begin{aligned}
P(k, s) & =\int_{2 s<|| \eta|-k|<1} \frac{|\eta|^{2}}{|\eta|^{2}-(k+s)^{2}} d|\eta| \\
& =\frac{k+s}{2} \int_{2 s<|| \eta|-k|<1} \frac{d|\eta|}{|\eta|-(k+s)}+\int_{2 s<|| \eta|-k|<1} \frac{2|\eta|+k+s}{2(|\eta|+k+s)} d|\eta| .
\end{aligned}
$$

As in the proof of Theorem 2.1, this suffices. 


\section{Section 3. Existence and Regularity of $h(\xi, \zeta, k)$}

The function $h(\xi, \zeta, k)$ on $\mathbf{R}^{3} \times \mathbf{R}^{3} \times \overline{\mathbf{R}}_{+}$is defined to be the solution of

$$
h(\xi, \zeta, k)+(2 \pi)^{-3} \int_{\mathbf{R}^{3}} \frac{\hat{q}(\xi-\eta) h(\eta, \zeta, k)}{|\eta|^{2}-(k+i 0)^{2}} d \eta=-\hat{q}(\xi-\zeta) .
$$

We will assume that $\hat{q} \in H_{\alpha, N}$ for some $\alpha$ and $N$. We will not assume that $\hat{q}$ is the Fourier transform of a real-valued function. In this situation one has the following existence theorem, considering $\zeta$ and $k$ as parameters.

Theorem 3.1. Given $(\alpha, N), 0<\alpha<1, N>1$, for all $\zeta \in \mathbf{R}^{3}$ and $k \geqq 0$, (3.1) has a unique solution $h(\xi, \zeta, k)$ such that $\Lambda_{\zeta}^{N}(\cdot) h(\cdot, \zeta, k) \in C^{\alpha}\left(\mathbf{R}^{3}\right)$, when $\hat{q}$ belongs to an open set $\mathcal{O}$ in $H_{\alpha, N}$. Moreover, the intersection of $\mathcal{O}$ with $H_{\alpha, N}^{r}=\left\{\hat{q} \in H_{\alpha, N}: \hat{q}(-\xi)=\overline{\hat{q}(\xi)}\right\}$ is dense in $H_{\alpha, N}^{r}$.

Remark 1. Note that $H_{\alpha, N}^{r}$ is simply the subspace of $H_{\alpha, N}$ (considered as vector space with real scalars) consisting of Fourier transforms of real-valued functions. The set $\mathcal{O}$ in this theorem is actually dense in $H_{\alpha, N}$ (see Remark 4 following the proof), but it is the stated density of $\mathcal{O} \cap H_{\alpha, N}^{r}$ in $H_{\alpha, N}^{r}$ that is important for our main results here.

Remark 2. One does not have existence for all real-valued $q \in C_{0}^{\infty}\left(\mathbf{R}^{3}\right)$, as the following family of examples shows. Let $u(x)$ be any positive function in $C^{\infty}\left(\mathbf{R}^{3}\right)$ such that $u(x)=|x|^{-1}$ for $|x|>R$, and define $q=\Delta u / u \in C_{0}^{\infty}\left(\mathbf{R}^{3}\right)$. Then $-\Delta u+q u=0$. Since $\left|D^{\alpha} u(x)\right| \leqq C_{\alpha}(1+|x|)^{-1-|\alpha|}$, for all $\alpha,|\hat{u}(\xi)| \leqq C_{k}|\xi|^{-k}$ for $|\xi|>1$ for all $k$. Moreover, since $u=|x|^{-1}+g, g$ supported in $|x| \leqq R, \hat{u}(\xi)=$ $-4 \pi|\xi|^{-2}+\hat{g}$, and $\hat{g}$ is entire. We have

$$
|\eta|^{2} \hat{u}(\eta)+(2 \pi)^{-3} \int_{\mathbf{R}^{3}} \hat{q}(\eta-\xi) \hat{u}(\xi) d \xi=0 .
$$

Assuming that (3.1) has a solution $h(\xi, 0,0) \in H_{\alpha, N}$ for $\zeta=0, k=0$ and taking the inner product with $\hat{u}(\xi)$ we conclude from (3.2) (note $\hat{q}(\xi-\eta)=\overline{\hat{q}}(\eta-\xi)$ )

$$
0=\int_{\mathbf{R}^{3}} \overline{\hat{u}}(\xi) \hat{q}(\xi) d \xi
$$

However, by Plancherel's theorem

$$
(2 \pi)^{-3} \int_{\mathbf{R}^{3}} \overline{\hat{u}}(\xi) \hat{q}(\xi) d \xi=\int_{\mathbf{R}^{3}} u(x) q(x) d x \equiv \int_{\mathbf{R}^{3}} \Delta u d x=-4 \pi .
$$

Throughout this section we will work with the modified operators and functions,

$$
\begin{aligned}
\tilde{A}(\hat{q}, \zeta, k) & =\Lambda_{\zeta}^{N} A(\hat{q}, k) \Lambda_{\zeta}^{-N}, \tilde{h}(\xi, \zeta, k)=\Lambda_{\zeta}^{N}(\xi) h(\xi, \zeta, k), \\
\tilde{q}(\xi) & =\Lambda^{N}(\xi) \hat{q}(\xi) \quad \text { and } \quad \tilde{q}_{\zeta}(\xi)=\Lambda_{\zeta}^{N}(\xi) \hat{q}(\xi-\zeta) .
\end{aligned}
$$

We will also frequently suppress some or all of the variables $\hat{q}, \xi, \zeta, k$ in $\tilde{A}$ and $\tilde{h}$. In this notation (3.1) becomes

$$
\tilde{h}(\xi, k, \zeta)+[\tilde{A}(\tilde{q}, \zeta, k) \tilde{h}(\cdot \zeta, k)](\xi)=-\tilde{q}_{\zeta}(\xi)
$$

or, more compactly

$$
\tilde{h}+\tilde{A} \tilde{h}=-\tilde{q}_{\zeta} .
$$


Proof of Theorem 3.1. Theorem 2.1 implies that for $\hat{q} \in H_{\alpha, N}, 0<\alpha^{\prime} \leqq \alpha$ and $0 \leqq \delta<\min \{1, N-1\}$,

$$
\left\|\tilde{A} \Lambda^{\delta} f\right\|_{\alpha} \leqq C\|f\|_{\alpha^{\prime}}
$$

Thus, since for $\alpha^{\prime}<\alpha$ and $\delta>0\left\{g:\|g\|_{\alpha} \leqq 1\right\}$ has compact closure in $C^{\alpha^{\prime}, \delta}=$ $\left\{g:\left\|\Lambda^{-\delta} g\right\|_{\alpha^{\prime}}<\infty\right\}$, we see that $\tilde{A}$ is a compact operator on $C^{\alpha}\left(\mathbf{R}^{3}\right)$. Hence, since $\tilde{q}_{\zeta}$ is in $C^{\alpha}\left(\mathbf{R}^{3}\right)$ by hypothesis, (3.3) is a Fredholm equation in $C^{\alpha}\left(\mathbf{R}^{3}\right)$ for $\tilde{h}$. We will prove the first part of Theorem 3.1 by showing that the set $\mathcal{O}$ of $\hat{q}$ such that $I+\widetilde{A}(\hat{q}, \zeta, k)$ has trivial kernel in $C^{\alpha}\left(\mathbf{R}^{3}\right)$ for all $(\zeta, k) \in \mathbf{R}^{3} \times \overline{\mathbf{R}}_{+}$is open. For $\hat{q} \in \mathcal{O}$ (3.3) has a unique solution $\tilde{h}$ in $C^{\alpha}\left(\mathbf{R}^{3}\right)$. Since $C(\zeta)^{-1} \leqq \Lambda^{N} \Lambda_{\zeta}^{-N} \leqq C(\zeta)$, one sees that $\Lambda_{\zeta}^{-N} \tilde{h}$ is the unique solution to (3.1) with $\Lambda^{N} h \in C^{\alpha}\left(\mathbf{R}^{3}\right)$.

Theorem 2.1 implies that given $\hat{q}_{0} \in H_{\alpha, N}$ the operator norm on $C^{\alpha}\left(\mathbf{R}^{3}\right)$, $\|\tilde{A}(\hat{q}, \zeta, k)\|_{\alpha}$ will be less than 1 , for $k>k_{0}$ and $\left\|\hat{q}-\hat{q}_{0}\right\|_{\alpha, N} \leqq 1$. Thus $I+\tilde{A}(\hat{q}, \zeta, k)$ is injective for $k>k_{0}$ and $\left\|\hat{q}-\hat{q}_{0}\right\|_{\alpha, N} \leqq 1$. Since $(C(\zeta))^{-1} \leqq \Lambda_{\zeta}^{-N} \Lambda^{N} \leqq C(\zeta)$, if $I+\widetilde{A}(\hat{q}, 0, k)$ is injective on $C^{\beta}\left(\mathbf{R}^{3}\right)$, then $I+\widetilde{A}(\hat{q}, \zeta, k)$ is injective on $C^{\beta}\left(\mathbf{R}^{3}\right)$ for all $\zeta \in \mathbf{R}^{3}$. Applying Theorem 2.2, we have

$$
|\Delta(s) \Delta(\mu) \tilde{A}(\hat{q}, 0, k) f| \leqq C s^{\alpha^{\prime}}\|\Delta(\mu) \hat{q}\|_{\alpha^{\prime}, N}\|f\|_{\alpha^{\prime}},
$$

where $\Delta(s)$ and $\Delta(\mu)$ are the difference operators in $k$ and $\xi$, respectively. Hence arguing as in the initial reduction in the proof of Theorem 2.1, we see for $\alpha^{\prime}=\alpha / 2$,

$$
\|\Delta(s) \tilde{A}(\hat{q}, 0, k) f\|_{\alpha / 2} \leqq C s^{\alpha / 2}\|\hat{q}\|_{\alpha, N}\|f\|_{\alpha / 2},
$$

uniformly for $k \geqq 0$. Thus, as an operator acting on $C^{\alpha / 2}\left(\mathbf{R}^{3}\right), \tilde{A}(\hat{q}, 0, k)$ is norm continuous in $(\hat{q}, k)$ with the topology of $H_{\alpha, N} \times \overline{\mathbf{R}}_{+}$.

Now suppose $I+\tilde{A}\left(\hat{q}_{0}, \zeta, k\right)$ has no nullspace in $C^{\alpha}\left(\mathbf{R}^{3}\right)$ for $(\zeta, k) \in \mathbf{R}^{3} \times \overline{\mathbf{R}}_{+}$. If $f+\widetilde{A}\left(\hat{q}_{0}, \zeta, k\right) f=0$ for some $f \in C^{\alpha / 2}\left(\mathbf{R}^{3}\right)$, then Theorem 2.1, implies $f \in C^{\alpha}\left(\mathbf{R}^{3}\right)$. Hence $I+\tilde{A}\left(\hat{q}_{0}, \zeta, k\right)$ has no nullspace in $C^{\alpha / 2}\left(\mathbf{R}^{3}\right)$ for $(\zeta, k) \in \mathbf{R}^{3} \times \overline{\mathbf{R}}_{+}$. Thus by the remarks in the preceding paragraph $I+\tilde{A}(\hat{q}, \zeta, k)$ is injective on $C^{\alpha / 2}\left(\mathbf{R}^{\mathbf{3}}\right)$ for $k \geqq k_{0}$ when $\left\|\hat{q}-\hat{q}_{0}\right\|_{\alpha, N}<1$ and injective on $C^{\alpha / 2}\left(\mathbf{R}^{3}\right)$ for $0 \leqq k \leqq k_{0}$ when $\left\|q-\hat{q}_{0}\right\|_{\alpha, N}<\varepsilon$ for some $\varepsilon>0$. Thus, the set of $\hat{q}$ for which $I+\tilde{A}(\hat{q}, \zeta, k)$ is injective on $C^{\alpha}\left(\mathbf{R}^{3}\right)$ is open in $H_{\alpha, N}$.

To verify the density assertion in Theorem 3.1 we consider real-valued $q \in C_{0}^{\infty}\left(\mathbf{R}^{3}\right)$. The Fourier transforms of these $q$ are easily seen to be dense in $H_{\alpha, N}^{r}$. If for $k>0, \hat{f}+A(\hat{q}, k) \hat{f}=0$ has a nontrivial solution with $\Lambda^{N} \hat{f} \in C^{\alpha}\left(\mathbf{R}^{3}\right)$, we set for $\varepsilon \geqq 0$,

$$
u_{\varepsilon}=\int_{\mathbf{R}^{3}} \frac{e^{i x \cdot \xi} \hat{f}(\xi) d \xi}{|\xi|^{2}-(k+i \varepsilon)^{2}}
$$

Note $u_{\varepsilon} \in L^{2}\left(\mathbf{R}^{3}\right)$ for $\varepsilon>0$, and, taking the inverse Fourier transform of $\hat{f}+A(\hat{q}, k) \hat{f}=0,-\Delta u_{0}+q u_{0}=k^{2} u_{0}$ which implies $q u_{0} \in C_{0}^{\infty}\left(\mathbf{R}^{3}\right)$. We also have $\left(-\Delta-(k+i \varepsilon)^{2}\right) u_{\varepsilon}+q u_{0}=0$, which implies

$$
u_{\varepsilon}(x)=\frac{-1}{4 \pi} \int_{\mathbf{R}^{3}} \frac{e^{i(k+i \varepsilon)|x-y|}}{|x-y|} q(y) u_{0}(y) d y,
$$


and hence

$$
u_{0}(x)=\frac{-1}{4 \pi} \int_{\mathbf{R}^{3}} \frac{e^{i k|x-y|}}{|x-y|} q(y) u_{0}(y) d y .
$$

Now standard arguments show $u_{0} \in L^{2}\left(\mathbf{R}^{3}\right)$ and hence $u_{0} \equiv 0$. Thus $I+A(\hat{q}, k)$ is injective for $k>0$, and $I+\tilde{A}(\hat{q}, 0, k)$ is invertible on $C^{\alpha}\left(\mathbf{R}^{3}\right)$ for $k>0$.

Suppose $I+\tilde{A}(\hat{q}, 0,0)$ has nontrivial nullspace for $\hat{q} \in S=\left\{\left\|\hat{q}-\hat{q}_{0}\right\|_{\alpha, N}<\delta\right\} \cap$ $H_{\alpha, N}^{r}$. Let $m=\operatorname{dim} \operatorname{Null}\left\{I+\tilde{A}\left(\hat{q}_{1}, 0,0\right)\right\}$ be minimal for $\hat{q} \in S$. Then $\operatorname{dim}$ Null $\{I+$ $\tilde{A}(\hat{q}, 0,0)\}=m$, for all $\hat{q}$ with $\left\|\hat{q}-\hat{q}_{1}\right\|_{\alpha, N}<\delta^{\prime}$, for some $\delta^{\prime}>0$. This follows from the continuity of the projection

$$
P(\hat{q})=\oint_{|z-1|=\varepsilon}(z I+\tilde{A}(\hat{q}, 0,0))^{-1} d z
$$

in $\hat{q}$ on a neighborhood of $\hat{q}_{1}$ for $\varepsilon$ sufficiently small. Moreover, for all $\hat{f} \in C^{\alpha}\left(\mathbf{R}^{3}\right)$,

$$
(I+\tilde{A}(\hat{q}, 0,0)) P(\hat{q}) \hat{f}=0
$$

for $\left\|\hat{q}-\hat{q}_{1}\right\|_{\alpha, N}<\delta^{\prime \prime}$. Let $\hat{q}(t)=\hat{q}_{1}+t \hat{q}, \hat{q} \in H_{\alpha, N}^{r}$. For $t$ sufficiently small, one sees by substituting the power series for $\left(z I+\tilde{A}\left(q_{1}, 0,0\right)+t \tilde{A}(\hat{q}, 0,0)\right)^{-1}$ into (3.5) that $P(\hat{q}(t))$ is analytic in $t$. Differentiating

$$
(I+\tilde{A}(\hat{q}(t), 0,0) P(\hat{q}(t)) \hat{f}=0
$$

with respect to $t$ at $t=0$, we have

$$
\left(I+\tilde{A}\left(\hat{q}_{1}, 0,0\right)\right) \dot{V}=-\tilde{A}(\hat{q}, 0,0) V,
$$

where $\dot{V}=d /\left.d t P(\hat{q}(t)) \hat{f}\right|_{t=0}$ and $V=P\left(\hat{q}_{1}\right) \hat{f}$. As in Remark 2, taking the inner product with $V(\xi)|\xi|^{-2} \Lambda^{-N}(\xi)=w(\xi)|\xi|^{-2}$,

$$
0=\int_{\mathbf{R}^{3}} \int_{\mathbf{R}^{3}} \frac{\hat{q}(\xi-\eta) w(\eta)}{|\eta|^{2}} \frac{\bar{w}(\xi)}{|\xi|^{2}} d \xi d \eta=(2 \pi)^{3} \int_{\mathbf{R}^{3}} q(x)|h|^{2} d x,
$$

where $h$ is the inverse Fourier transform of $w(\xi)|\xi|^{-2}$. Since we can choose $\hat{f}$ so that $w \neq 0$ and $q$ is arbitrary, this is a contradiction.

Finally we note that, since the Fourier transform $\hat{R}$ of the set $R$ of real-valued $q \in C_{0}^{\infty}\left(\mathbf{R}^{3}\right)$ is dense in $H_{\alpha, N}^{r}$, if $I+\hat{A}(\hat{q}, 0,0)$ has a nontrivial nullspace for all $\hat{q} \in \hat{R} \cap S$ it must have a nontrivial nullspace for all $\hat{g} \in S$. This follows from the compactness of $\tilde{A}(\hat{q}, 0,0)$ for $\hat{q} \in H_{\alpha, N}$ and its continuity in $\hat{q}$. Thus the preceding contradiction shows that given $\hat{q}_{1} \in \hat{R}$ there is no $\delta$ such that $I+\hat{A}(\hat{q}, 0,0)$ has a nontrivial nullspace for $\hat{q} \in \hat{R} \cap\left\{\left\|\hat{q}-\hat{q}_{1}\right\|_{\alpha, N}<\delta\right\}$. Thus we conclude $I+\widetilde{A}(\hat{q}, \zeta, k)$ is injective for $\hat{q}$ in a dense subset of $\hat{R}$.

Remark 3. The computations following (3.5) are much more transparent in $x$-space. In $x$-space the equation $(I+A(\hat{q}, 0)) \hat{f}=0$, becomes

$$
\left(I+q E_{0}\right) f=0
$$

where $E_{0}$ is the operator

$$
\left[E_{0} g\right](x)=\frac{1}{4 \pi} \int_{\mathbf{R}^{3}} \frac{g(y)}{|x-y|} d y .
$$


Setting $q=q(t)$ and $f=f(t)$ and differentiating in $t$, we have

$$
\left(I+q E_{0}\right) \dot{f}=-\dot{q} E_{0} f .
$$

Since $q$ is real,

$$
\int_{\mathbf{R}^{3}} \overline{E_{0} f}\left(I+q E_{0}\right) g d x=\int_{\mathbf{R}^{3}} \overline{\left(f+q E_{0} f\right)}\left(E_{0} g\right) d x=0
$$

for all $g$. Thus

$$
0=\int_{\mathbf{R}^{3}} \dot{q}\left|E_{0} f\right|^{2} d x
$$

We work in $\xi$-space in the proof of Theorem 3.1 and elsewhere because we have no simple characterization of the inverse Fourier transform of $H_{\alpha, N}$.

Remark 4. Though our interest here is primarily in potentials with small imaginary parts, it is not at all difficult to extend the arguments used to prove Theorem 3.1 to show that the set of complex potentials $q$ in $C_{0}^{\infty}\left(\mathbf{R}^{3}\right)$ such that $\hat{q} \in \mathcal{O}$ is large enough that $\mathcal{O} \cap H_{\alpha, N}$ is a dense, open subset of $H_{\alpha, N}$. A sketch of one way to do this follows.

Given $q \in C_{0}^{\infty}(|x|<R)$, if $\hat{f} \in H_{\alpha, N}$ and $\hat{f}+A(\hat{q}, k) \hat{f}=0$, then $f \in C_{0}^{\infty}(|x|<R)$ and $k \leqq k_{0}\left(\|\hat{q}\|_{\alpha, N}\right)$ (by Theorem 2.1). Thus, taking $s$ large enough that $\|\hat{q}\|_{\alpha, N} \leqq C\|q\|_{s}$, where \|\|$_{s}$ is the norm on the Sobolev space $H_{s}(|x|<R)$, to show the injectivity of $I+A(\hat{q}, k)$ on $H_{\alpha, N}$ when $k \geqq 0$ for a dense set of $\hat{q}$ in $H_{\alpha, N}$, it will suffice to show that for any $R, I+q E_{z}$ is injective on $L^{2}(|x|<R)$ for $z \geqq 0$ for a dense set of $q$ in $\stackrel{\circ}{H}_{s}(|x|<R)$, where

$$
E_{z} f=\frac{1}{4 \pi} \int_{|x|<R} \frac{e^{i z|x-y|}}{|x-y|} f(y) d y .
$$

Given $q_{0} \in C_{0}^{\infty}(|x|<R)$, since $q_{0} E_{z}$ is both compact and entire in $z$ as an operator on $L^{2}(|x|<R)$ and $I+q_{0} E_{z}$ is injective for $z \gg 0,\left(I+q_{0} E_{z}\right)^{-1}$ is meromorphic with only a finite number of poles $k_{1}, \ldots, k_{M}$ on $k \geqq 0$. Using contour integrals to define projections on the nullspaces of $I-q_{0} E_{k_{j}}$ as in the proof of Theorem 3.1, one can get $\varepsilon>0$ and functions $\lambda_{j}(q, z)$ analytic on $D_{j}=\left\{\left\|q-q_{0}\right\|_{s}<\varepsilon,\left|z-k_{j}\right|<\varepsilon\right\}$, $j=1, \ldots, M$, such that, for $\left\|q-q_{0}\right\|_{s}<\varepsilon$ and $\left|z-k_{j}\right|<\varepsilon, I+q E_{z}$ fails to be injective if and only if $\lambda_{j}(q, z)=0$.

For each $j$ an argument similar to the one given in the proof of Theorem 3.1 shows $\lambda_{j}\left(q, k_{j}\right) \neq 0$. Thus one can choose $h \in C_{0}^{\infty}(|x|<R)$ such that for $j=1, \ldots, M$,

$$
d_{j}(w, z)=\lambda_{j}\left(q_{0}+w h, z\right)
$$

is an analytic function on $\left\{|w|<\varepsilon^{\prime},\left|z-k_{j}\right|<\varepsilon^{\prime}\right\}$ such that $\left(\partial^{p} d_{j} / \partial w^{p}\right)\left(0, k_{j}\right)=0$ for $p<N_{j}$ and

$$
\frac{\partial^{N_{J}} d_{j}}{\partial w^{N_{j}}}\left(0, k_{j}\right) \neq 0
$$

for some $N_{j}>0$. By the Weierstrass preparation theorem, for each $j$

$$
d_{j}(w, z)=\left(w^{N_{J}}+a_{1}(z) w^{N_{j-1}}+\cdots+a_{N_{j}}(z)\right) r(w, z)
$$


where $r\left(0, k_{j}\right) \neq 0$. Thus the zero set of $d_{j}$ in $\left\{|w|<\varepsilon^{\prime}<\varepsilon,\left|z-k_{j}\right|<\varepsilon^{\prime}<\varepsilon\right\} \cap\{$ real $z\}$ is the union of a finite set of curves $\left(w_{l}(k), k\right)$ where either $w_{l} \equiv 0$ or

$$
w_{l}(k)=a_{l}\left(k-k_{j}\right)^{r_{l}}+o\left(\left(k-k_{j}\right)^{r_{l}}\right.
$$

with $a_{l} \neq 0$ and $r_{l}$ rational. Thus we can choose $w_{n} \rightarrow 0$ such that $d_{j}\left(w_{n}, k\right) \neq 0$ for $\left|k-k_{j}\right|<\varepsilon^{\prime}, j=1, \ldots, M$ for all $n$. Since $I+q_{0} E_{k}$ is injective for $k \neq k_{j}$ and $w_{n} \rightarrow 0$, we see that $I+\left(q_{0}+w_{n} h\right) E_{k}$ is injective for all $k \geqq 0$ for $n>n_{0}$.

Our estimates on the regularity and growth of $h(\xi, \zeta, k)$ are primarily directed toward showing that the backscattering amplitude $h(\xi,-\xi,|\xi|)$ belongs to $H_{\alpha, N}$ when $\hat{q}$ is in the set $\mathcal{O} \subset H_{\alpha, N}$ of Theorem 3.1. However, the expression we use for the Frechet derivative of the backscattering map $\hat{q}(\xi) \rightarrow h(\xi,-\xi,|\xi|)$ involves $h(\xi, \zeta,|\zeta|)$, and it is actually easier to treat $\xi, \zeta, k$ as independent variables. Thus our estimate takes the following form.

Theorem 3.2. Let $\mathcal{O}$ be the open subset of $H_{\alpha, N}$ in Theorem 3.1, i.e. let $\mathcal{O}$ be the set of $\hat{q} \in H_{\alpha, N}$ such that $I+\tilde{A}(\hat{q}, \zeta, k)$ in injective on $C^{\alpha}\left(\mathbf{R}^{3}\right)$ for all $(\zeta, k) \in \mathbf{R}^{3} \times \overline{\mathbf{R}}_{+}$. Then, for $\hat{q} \in \mathcal{O}, h(\xi, \zeta, k)$ satisfies

$$
\left\|\Lambda_{\zeta}^{N} h\right\|_{\underline{\alpha}}<\infty \text {. }
$$

Here \|\|$_{\alpha}$ is the norm on functions on $\mathbf{R}^{3} \times \mathbf{R}^{3} \times \overline{\mathbf{R}}_{+}$introduced in (1.1).

Proof. From Theorem 3.1 we know that $(I+\tilde{A}(\hat{q}, \zeta, k))^{-1}$, and hence $\tilde{h}$ exist for $\hat{q} \in \mathcal{O}$. However, here we want to show that $\sup \|\tilde{h}(\cdot, \zeta, k)\|_{\alpha}<\infty$. For this we will show that

$$
\sup _{\zeta, k}\left\|\left(I+\Lambda^{-\delta / 2} \tilde{A}(\hat{q}, \zeta, k) \Lambda^{\delta / 2}\right)^{-1}\right\|_{\alpha / 2}<\infty .
$$

Note that Theorem 2.1 implies that if $f+\Lambda^{-\delta / 2} \tilde{A} \Lambda^{\delta / 2} f=0$ and $f \in C^{\alpha / 2}\left(\mathbf{R}^{3}\right)$, then $\Lambda^{\delta / 2} f \in C^{\alpha}\left(\mathbf{R}^{3}\right)$. Hence $I+\Lambda^{-\delta / 2} \tilde{A} \Lambda^{\delta / 2}$ is injective on $C^{\alpha / 2}\left(\mathbf{R}^{3}\right)$ for $\hat{q} \in \mathcal{O}$. Moreover, $\Lambda^{-\delta / 2} \tilde{A} \Lambda^{\delta / 2}$ is compact on $C^{\alpha / 2}\left(\mathbf{R}^{3}\right)$ by the argument used in the proof of Theorem 3.1. Thus $I+\Lambda^{-\delta / 2} \tilde{A}(\hat{q}, \zeta, k) \Lambda^{\delta / 2}$ is invertible on $C^{\alpha / 2}\left(\mathbf{R}^{3}\right)$ for $(\zeta, k) \in \mathbf{R}^{3} \times \overline{\mathbf{R}}_{+}$for $\hat{q} \in \mathcal{O}$. Using Theorems 2.1 and 2.2 as in the proof of (3.4), one has uniformly for $(\zeta, k) \in \mathbf{R}^{3} \times \overline{\mathbf{R}}_{+}$,

$$
\left\|\Lambda^{-\delta / 2}(\tilde{A}(\hat{q}, \zeta, k+s)-\tilde{A}(\hat{q}, \zeta, k)) \Lambda^{\delta / 2}\right\|_{\alpha / 2} \leqq C s^{\alpha / 2}
$$

Moreover, simply by using (2.5) we can extend (3.7) to

$$
\left\|\Lambda^{-\delta / 2}(\tilde{A}(\hat{q}, \zeta+\mu, k+s)-\tilde{A}(\hat{q}, \zeta, k)) \Lambda^{\delta / 2}\right\|_{\alpha / 2} \leqq C\left(s^{\alpha / 2}+|\mu|\right),
$$

where $C$ is independent of $\zeta$ and $k$.

Since Theorem 2.1 implies that $\left\|\Lambda^{-\delta / 2} \tilde{A}(\hat{q}, \zeta, k) \Lambda^{\delta / 2}\right\|_{\alpha / 2} \leqq 1 / 2$ for $k>k(\hat{q})$ for all $\zeta \in \mathbf{R}^{3}$, we can use the Neumann series representation of $\left(I+\Lambda^{-\delta / 2} \tilde{A}(\hat{q}, \zeta, k) \Lambda^{\delta / 2}\right)^{-1}$ to conclude that

$$
\left\|\left(I+\Lambda^{-\delta / 2} \tilde{A}(\hat{q}, \zeta, k) \Lambda^{\delta / 2}\right)^{-1}\right\|_{\alpha / 2} \leqq C
$$

for $\zeta \in \mathbf{R}^{3}, k \geqq k(\hat{q})$. Since for any invertible operators $I+B$ and $I+B_{0}$,

$$
(I+B)^{-1}-\left(I+B_{0}\right)^{-1}=(I+B)^{-1}\left(B-B_{0}\right)\left(I+B_{0}\right)^{-1},
$$


the estimate (3.8) implies

$$
\left\|\left(I+\Lambda^{-\delta / 2} \tilde{A}(\zeta, k) \Lambda^{\delta / 2}\right)^{-1}\right\|_{\alpha / 2} \leqq 2\left\|\left(I+\Lambda^{-\delta / 2} \tilde{A}\left(\zeta_{0}, k_{0}\right) \Lambda^{\delta / 2}\right)^{-1}\right\|_{\alpha / 2}
$$

for $\left|k-k_{0}\right|+\left|\zeta-\zeta_{0}\right|<\varepsilon_{0}$. Thus, for any $R<\infty$,

$$
\left\|\left(I+\Lambda^{-\delta / 2} \tilde{A}(\hat{q}, \zeta, k) \Lambda^{\delta / 2}\right)^{-1}\right\|_{\alpha / 2} \leqq C_{R}
$$

for $0 \leqq k \leqq k(\hat{q}),|\zeta| \leqq R$.

To bound $\left\|\left(I+\Lambda^{-\delta / 2} \tilde{A}(\hat{q}, \zeta, k) \Lambda^{\delta / 2}\right)^{-1}\right\|_{\alpha / 2}$ as $|\zeta| \rightarrow \infty$, we will begin by showing that

$$
\left\|\Lambda^{-\delta / 2}(\tilde{A}(\hat{q}, \zeta, k)-A(\hat{q}, k)) \Lambda^{\delta / 2}\right\|_{\alpha / 2} \rightarrow 0
$$

as $|\zeta| \rightarrow \infty$, uniformly in $k$ and $\hat{q}$ on bounded sets of $\hat{q}$.

Given $\varphi \in C_{0}^{\infty}\left(\mathbf{R}^{3}\right)$ with $\varphi(\xi)=1$ for $|\xi| \leqq 1$, one sees easily that the operator norm of multiplication by $(1-\varphi(\xi / R)) \Lambda^{-\delta / 2}(\xi)$ on $C^{\alpha / 2}\left(\mathbf{R}^{3}\right)$ tends to zero as $R \rightarrow \infty$. Since Theorem 2.1 implies $\left\|\tilde{A}(\hat{q}, \zeta, k) \Lambda^{\delta}\right\|_{\alpha / 2} \leqq C\|\hat{q}\|_{\alpha, N}$ for $(\zeta, k) \in \mathbf{R}^{3} \times \overline{\mathbf{R}}_{+}$, we see, letting $\varphi_{R}(\xi)=\varphi(\xi / R)$,

and

$$
\left\|\left(1-\varphi_{R}\right) \Lambda^{-\delta / 2} \tilde{A}(\hat{q}, \zeta, k) \Lambda^{\delta / 2}\right\|_{\alpha / 2} \rightarrow 0
$$

$$
\left\|\Lambda^{-\delta / 2} \tilde{A}(\hat{q}, \zeta, k) \Lambda^{\delta / 2}\left(1-\varphi_{R}\right)\right\|_{\alpha / 2} \rightarrow 0
$$

as $R \rightarrow \infty$ uniformly in $(\zeta, k)$ on bounded sets of $\hat{q}$.

To obtain the estimate $\left\|A(\hat{q}, k) \Lambda^{\delta}\right\|_{\alpha / 2} \leqq C\|\hat{q}\|_{\alpha, N}$, we must repeat the derivation of the bounds on $\left|I_{1}\right|,\left|I_{2}\right|$ and $\left|I_{3}\right|$ without the weight factor $\Lambda_{\zeta}^{N}(\xi) / \Lambda_{\zeta}^{N}(\eta)$. We have

$$
\left|I_{1}\right| \leqq C\|f\|_{0}\|\hat{q}\|_{0, N} \int_{|| \eta \mid} \frac{\left(1+|\eta|^{2}\right)^{\delta / 2}}{\left.\left(1+|\xi-\eta|^{2}\right)^{N / 2}|| \eta\right|^{2}-k^{2} \mid} d \eta,
$$

and as in the proof of (2.2), this implies

$$
\left|I_{1}\right| \leqq C\|f\|_{0}\|\hat{q}\|_{0, N} \int_{|| \eta \mid} \frac{\left(1+|\eta|>\left.1\right|^{2}\right)^{(\delta-\beta) / 2}}{\left.|| \eta\right|^{2}-k^{2} \mid}|\eta|^{2} d|\eta|
$$

with $\beta>1+\delta$. Hence $\left|I_{1}\right| \leqq C\|f\|_{0}\|\hat{q}\|_{0, N}$. For $I_{2}$ we have

$$
\left|I_{2}\right| \leqq C\|f\|_{\alpha / 2}\|\hat{q}\|_{\alpha / 2, N} \int_{|| \eta|-k| \leqq 1} \frac{\left(1+|\eta|^{2}\right)^{\delta / 2}\left(1+|\xi-\eta|^{2}\right)^{-N / 2}}{(|\eta|+k)|| \eta|-k|^{1-\alpha / 2}} d \eta
$$

so that $\left|I_{2}\right| \leqq C\|f\|_{\alpha / 2}\|\hat{q}\|_{\alpha / 2, N}$. Likewise,

$$
\left|I_{3}\right| \leqq C\|f\|_{0}\|\hat{q}\|_{0, N} .
$$

Thus we conclude $\sup _{\xi}\left|\left[A(\hat{q}, k) \Lambda^{\delta} f\right](\xi)\right| \leqq C\|f\|_{\alpha / 2}\|\hat{q}\|_{\alpha / 2, N}$. Then, since $\Delta(\mu)$ $(A(\hat{q}, k) f)=A(\Delta(\mu) \hat{q}, k) \stackrel{\xi}{f}$, we have

$$
\left\|A(\hat{q}, k) \Lambda^{\delta} f\right\|_{\alpha / 2} \leqq C\|\hat{q}\|_{\alpha, N}\|f\|_{\alpha / 2},
$$

uniformly in $k$ as desired. Thus

and

$$
\left\|\left(1-\varphi_{R}\right) \Lambda^{-\delta / 2} A(\hat{q}, k) \Lambda^{\delta / 2}\right\|_{\alpha / 2} \rightarrow 0
$$

$$
\left\|\Lambda^{-\delta / 2} A(\hat{q}, k) \Lambda^{\delta / 2}\left(1-\varphi_{R}\right)\right\|_{\alpha / 2} \rightarrow 0
$$


as $R \rightarrow \infty$ uniformly in $k$ on bounded sets of $\hat{q}$.

Next we consider

$$
\varphi_{R} \Lambda^{-\delta / 2}(\tilde{A}(\hat{q}, \zeta, k)-A(\hat{q}, k)) \Lambda^{\delta / 2} \varphi_{R} .
$$

We view this as a modification of the operator $\tilde{A}(q, \zeta, k)$ in which the weight factor $\omega(\xi, \eta, \zeta)=\Lambda_{\zeta}^{N}(\xi) \Lambda_{\zeta}^{-N}(\eta)$ has been replaced by

$$
\omega_{R}(\xi, \eta, \xi)=\varphi_{R}(\xi) \Lambda^{-\delta / 2}(\xi)\left(1-\Lambda_{\zeta}^{N}(\eta) \Lambda_{\zeta}^{-N}(\xi)\right) \Lambda^{\delta / 2}(\eta) \varphi_{R}(\eta) \omega(\xi, \eta, \zeta) .
$$

Since for any $M, \Lambda_{\zeta}^{N}(\eta) \Lambda_{\zeta}^{-N}(\xi) \rightarrow 1$ uniformly on $\{|\xi|<M,|\eta|<M\}$ as $|\zeta| \rightarrow \infty$, given $\varepsilon$, we have $\omega_{R}(\xi, \eta, \zeta) \leqq \varepsilon \omega(\xi, \eta, \zeta)$ for $|\zeta|>C(R)$. Likewise, letting $\Delta(\mu)$ denote the difference operator in $\xi$ or $\eta,(1 /|\mu|) \Delta(\mu)\left(\Lambda_{\zeta}^{N}(\eta) \Lambda_{\zeta}^{-N}(\xi)\right) \rightarrow 0$ uniformly on $\{|\xi|<M,|\eta|<M\}$ as $|\zeta| \rightarrow \infty$, and we have $\left|\Delta(\mu) \omega_{R}(\xi, \eta, \zeta)\right|<\varepsilon|\mu| \omega(\xi, \eta, \zeta)$ for $|\zeta|>C(R)$. In the proof of Theorem 2.1 we only used

$$
\omega(\xi, \eta, \zeta) \leqq \Lambda_{\zeta}^{N}(\xi) \Lambda_{\zeta}^{-N}(\eta)
$$

and

$$
|\Delta(\mu) \omega(\xi, \eta, \zeta)| \leqq C|\mu| \Lambda_{\zeta}^{N}(\xi) \Lambda_{\zeta}^{-N}(\eta)
$$

Thus for $|\zeta|>C(R)$

$$
\left\|\varphi_{R} \Lambda^{-\delta / 2}(\tilde{A}(\hat{q}, \zeta, k)-A(\hat{q}, k)) \Lambda^{-\delta / 2} \varphi_{R}\right\|_{\alpha / 2} \leqq \varepsilon\|\hat{q}\|_{\alpha, N} .
$$

Combining (3.12), with the previous estimates on terms with factors of $\left(1-\varphi_{R}\right)$ yields (3.11).

From (3.11) we conclude that $\Lambda^{-\delta / 2} A(\hat{q}, k) \Lambda^{\delta / 2}$ is a compact operator-valued function on $C^{\alpha / 2}\left(\mathbf{R}^{3}\right)$ which is norm continuous in $(k, \hat{q})$. Thus to conclude that $\left\|\left(I+\Lambda^{-\delta / 2} \tilde{A}(\hat{q}, \zeta, k) \Lambda^{\delta / 2}\right)^{-1}\right\|_{\alpha / 2}$ is uniformly bounded for $0 \leqq k \leqq k(\hat{q})$ and $|\zeta|>R, R$ sufficiently large, we only need to show that $I+\Lambda^{-\delta / 2} A(\hat{\hat{q}}, k) \Lambda^{\delta / 2}$ is injective on $C^{\alpha / 2}\left(\mathbf{R}^{3}\right)$ for $0 \leqq k \leqq k(\hat{q})$. Note that $f+\Lambda^{-\delta / 2} A(\hat{q}, k) \Lambda^{\delta / 2} f=0$ implies $\tilde{f}+\Lambda^{-\delta / 2} \tilde{A}(\hat{q}, 0, k) \Lambda^{\delta / 2} \tilde{f}=0$, where $\tilde{f}=\Lambda^{N} f$. Hence, since $\hat{q} \in \mathcal{O}$, to complete the proof of (3.6) we only need the following.

Lemma 3.3. Assume $f+\Lambda^{-\delta / 2} A(\hat{q}, k) \Lambda^{\delta / 2} f=0, f \in C^{\alpha / 2}\left(\mathbf{R}^{3}\right)$ and $\hat{q} \in H_{\alpha, N}$. Then $\Lambda^{N+\delta / 2} f$ is in $C^{\alpha / 2}\left(\mathbf{R}^{3}\right)$. Here $0 \leqq \delta<\min \{1, N-1\}$ as before.

Proof of Lemma 3.3. We only need consider $f(\xi)$ when $|\xi|>k+1$. Then we have

$$
\begin{aligned}
|f(\xi)|= & \left|\Lambda^{-\delta / 2}(\xi)\left[A(\hat{q}, k) \Lambda^{\delta / 2} f\right](\xi)\right| \\
& =\left|\int_{|\eta|<k+1} \frac{\Lambda^{-\delta / 2}(\xi) \hat{q}(\xi-\eta) \Lambda^{\delta / 2}(\eta) f(\eta)}{|\eta|^{2}-(k+i 0)^{2}} d \eta\right|+C\|\hat{q}\|_{0, N} \\
& \equiv \int_{k+1<|\eta|<\infty}\left(1+|\eta-\xi|^{2}\right)^{-N / 2}\left(1+|\eta|^{2}\right)^{-1+\delta / 4}\left(1+|\xi|^{2}\right)^{-\delta / 4}|f(\eta)| d \eta \\
& \equiv I_{1}+I_{2} .
\end{aligned}
$$

We have $\left|I_{1}\right| \leqq C\left(1+|\xi|^{2}\right)^{-N / 2-\delta / 4}$, since $\left\|\Lambda^{N} \hat{q}\right\|_{\alpha}<\infty$, and the proof will proceed by repeated application of the inequality

$$
|f(\xi)| \leqq I_{2}+C\left(1+|\xi|^{2}\right)^{-N / 2-\delta / 4} .
$$


Assume that we have shown $|f(\xi)| \leqq C\left(1+|\xi|^{2}\right)^{-r / 2}$ for some $r \geqq 0$. Then

$$
I_{2} \leqq C \int_{k+1<|\eta|<\infty}\left(1+|\eta-\xi|^{2}\right)^{-N / 2}\left(1+|\eta|^{2}\right)^{\delta / 4-r / 2-1}\left(1+|\xi|^{2}\right)^{-\delta / 4} d \eta .
$$

We divide the region of integration into

$$
k+1<|\eta|<\frac{1}{2}|\xi| \text { and } \frac{1}{2}|\xi|<|\eta|<\infty
$$

getting $J_{1}$, and $J_{2}$. We have

$$
J_{1} \leqq \begin{cases}C\left(1+|\xi|^{2}\right)^{-N / 2}\left(1+|\xi|^{2}\right)^{(1-r) / 2} \ln |\xi|, & r \leqq 1+\delta / 2 \\ C\left(1+|\xi|^{2}\right)^{-N / 2-\delta / 4}, & r>1+\delta / 2\end{cases}
$$

Since

$$
\int_{S^{2}}\left(1+|| \eta|\omega-\xi|^{2}\right)^{-N / 2} d \omega \leqq \begin{cases}C|\eta|^{-2}, & N>2, \\ C|\eta|^{-2} \ln |\eta|, & N=2, \\ C|\eta|^{-N}, & 1<N<2,\end{cases}
$$

(see proof Lemma 2.2), and $0<\delta<\min \{1, N-1\}$, we have

$$
J_{2} \leqq\left(1+|\xi|^{2}\right)^{1 / 2-(r+\beta) / 2},
$$

where $\beta$ is defined as in the proof of Lemma 2.3. Thus repeated use (3.13) gives

$$
\mid f(\xi) \leqq C\left(1+|\xi|^{2}\right)^{-N / 2-\delta / 4}
$$

To show that

$$
|\Delta(\mu) f(\xi)| \leqq C|\mu|^{\alpha}\left(1+|\xi|^{2}\right)^{-N / 2-\delta / 4},
$$

one merely notes that

$$
|\Delta(\mu) \hat{q}(\xi-\eta)| \leqq C|\mu|^{\alpha}\|\hat{q}\|_{\alpha, N}\left(1+|\xi-\eta|^{2}\right)^{-N / 2}
$$

and uses the preceding estimates with $r=N+\delta / 2$.

Continuation of the Proof of Theorem 3.2. Since from (3.3) one has

$$
\Lambda^{-\delta / 2} \tilde{h}=-\left(I+\Lambda^{-\delta / 2} \tilde{A} \Lambda^{\delta / 2}\right)^{-1} \Lambda^{-\delta / 2} \tilde{q}_{\zeta}
$$

(3.6) implies

$$
\left\|\Lambda^{-\delta / 2}(\cdot) \tilde{h}(\cdot \zeta, k)\right\|_{\alpha / 2} \leqq C\left\|\Lambda^{-\delta / 2} \tilde{q}_{\zeta}\right\|_{\alpha / 2} \leqq C\left\|\tilde{q}_{\zeta}\right\|_{\alpha},
$$

where $C$ is independent of $(\zeta, k) \in \mathbf{R}^{3} \times \overline{\mathbf{R}}_{+}$. Now, writing $\tilde{h}=-\tilde{q}_{\zeta}-\tilde{A} \Lambda^{\delta / 2} \Lambda^{-\delta / 2} \tilde{h}$, and using Theorem 2.1, we have

$$
\sup _{\zeta, k}\|\tilde{h}(\cdot, \zeta, k)\|_{\alpha} \leqq C\left\|\tilde{q}_{\zeta}\right\|_{\alpha}=C\|\hat{q}\|_{\alpha, N} .
$$

Since $\tilde{h}=\Lambda_{\zeta}^{N} h, \sup _{\zeta, k}\|\tilde{h}(\cdot, \zeta, k)\|_{\alpha}$ is the first of the three norms in (1.1) whose sum is $\left\|\Lambda_{\zeta}^{N} h\right\|_{\underline{\alpha}}$. Note that, if we replace $\tilde{q}_{\zeta}$ by an arbitrary element of $C^{\alpha}\left(\mathbf{R}^{3}\right),(3.14)$ shows

$$
\left.\sup _{(\zeta, k)} \| I+\tilde{A}(\hat{q}, \zeta, k)\right)^{-1} \|_{\alpha}<\infty, \quad \text { for } \quad \hat{q} \in \mathcal{O} \text {. }
$$

Since Theorem 2.1 fails for $\alpha=0$, we cannot obtain estimates on $\|\tilde{h}(\xi, ;, k)\|_{\alpha}$ 
and $\|\tilde{h}(\xi, \zeta, \cdot)\|_{\alpha}$ by applying difference operators to (3.3). Instead we use the following procedure. Since $\hat{q}$ can be approximated in \|\|$_{\alpha, N}$ by $\hat{q}_{\infty} \in C_{0}^{\infty}\left(\mathbf{R}^{3}\right)$, we have

$$
\tilde{h}+\tilde{A}\left(\hat{q}_{1}, \zeta, k\right) \tilde{h}+\tilde{A}\left(\hat{q}_{\infty}, \zeta, k\right) \tilde{h}=-\tilde{q}_{\zeta}
$$

with $\left\|\hat{q}_{1}\right\|_{\alpha, N}<\varepsilon_{0}, \varepsilon_{0}$ to be chosen small enough that the Neumann series for $\left(I+\tilde{A}\left(\hat{q}_{1}, \zeta, k\right)\right)^{-1}$ converges. Then we set

$$
\tilde{q}_{\zeta}+\tilde{h}+\tilde{A}\left(\hat{q}_{1}, \zeta, k\right) \tilde{h}=\tilde{g},
$$

so that (3.15) becomes

$$
\tilde{g}+\tilde{A}\left(\hat{q}_{\infty}, \zeta, k\right)\left(I+\tilde{A}\left(\hat{q}_{1}, \zeta, k\right)\right)^{-1}\left(\tilde{g}-\tilde{q}_{\zeta}\right)=0 .
$$

The extra regularity of $\hat{q}_{\infty}$ and the explicit representation of $\left(I+\tilde{A}\left(\hat{q}_{1}, \zeta, k\right)\right)^{-1}$ via Neumann series will permit us to get regularity results for $\tilde{g}$ by applying difference operators to (3.16), and then pass to $\tilde{h}$ via

$$
\tilde{h}=\left(I+\tilde{A}\left(\hat{q}_{1}, \zeta, k\right)\right)^{-1}\left(\tilde{g}-\tilde{q}_{\zeta}\right) .
$$

The Neumann series expansion of $(I+A(\hat{q}, k))^{-1} f$ is given by

$$
(I+A(\hat{q}, k))^{-1} f=f+\sum_{n=1}^{\infty}(-1)^{n} A^{n}(q, k) f,
$$

where

$$
\left[A^{n} f\right](\xi, k)=\int_{R^{3 n}} \frac{\hat{q}\left(\xi-\eta_{1}\right) \hat{q}\left(\eta_{1}-\eta_{2}\right) \cdots \hat{q}\left(\eta_{n-1}-\eta_{n}\right) f\left(\eta_{n}\right)}{\prod_{j=1}^{n}\left(\left|\eta_{j}\right|^{2}-(k+i 0)^{2}\right)} d \eta_{1} \cdots d \eta_{n} .
$$

Expanding $\Delta(s) A^{n} f=\left[A^{n} f\right](\xi, k+s)-\left[A^{n} f\right](\xi, k)$ by Leibnitz' formula, we have

$$
\Delta(s) A^{n} f=\sum_{p=1}^{n} \int_{\mathbf{R}^{3}} \Delta(s)\left(\frac{1}{\left|\eta_{p}\right|^{2}-(k+i 0)^{2}}\right) Q_{p}\left(\xi, \eta_{p}\right) R_{p}\left(\eta_{p}\right) d \eta_{p},
$$

where for $p>1$,

$$
Q_{p}\left(\xi, \eta_{p}\right)=\int_{R^{3(p-1)}} \frac{\hat{q}\left(\xi-\eta_{1}\right) \cdots \hat{q}\left(\eta_{p-1}-\eta_{p}\right)}{\prod_{j=1}^{p-1}\left(\left|\eta_{j}\right|^{2}-(k+s+i 0)^{2}\right)} d \eta_{1}, \ldots, d \eta_{p-1},
$$

and for $p<n$

$$
R_{p}\left(\eta_{p}\right)=\int_{R^{3(n-p)}} \frac{\hat{q}\left(\eta_{p}-\eta_{p+1}\right) \cdots \hat{q}\left(\eta_{n-1}-\eta_{n}\right) f\left(\eta_{n}\right)}{\prod_{j=p+1}^{n}\left(\left|\eta_{j}\right|^{2}-(k+i 0)^{2}\right)} d \eta_{p+1} \cdots d \eta_{n}
$$

with $Q_{1}=\hat{q}\left(\xi-\eta_{1}\right)$ and $R_{n}=f\left(\eta_{n}\right)$. Applying Theorem 2.2 with $\delta=0$ and then Theorem 2.1 with $\delta=0$, we have

$$
\sup _{0<s<1}\left|\Lambda_{\zeta}^{N}(\xi) s^{-\alpha} \Delta(s) A^{n} f\right| \leqq C \sum_{p=1}^{n}\left\|\Lambda_{\zeta}^{N} Q_{p}\right\|_{\alpha}\left\|\Lambda_{\zeta}^{N} R_{p}\right\|_{\alpha} \leqq C\left(n \widetilde{C}^{n}\right)\left\|\Lambda^{N} \hat{q}\right\|_{\alpha}^{n}\left\|\Lambda_{\zeta}^{N} f\right\|_{\alpha}
$$


where $\tilde{C}$ is the constant from Theorem 2.1. Combining (3.19) with the direct estimate from Theorem 2.1,

$$
\left\|\Lambda_{\zeta}^{N}(\cdot)\left[A^{n} f\right](\cdot)\right\|_{\alpha} \leqq \widetilde{C}^{n}\left\|\Lambda^{N} \hat{q}\right\|_{\alpha}^{n}\left\|\Lambda_{\zeta}^{N} f\right\|_{\alpha},
$$

we conclude that, given $g \in C^{\alpha}\left(\mathbf{R}^{3}\right)$, the Neumann series expansion of

$$
\Lambda_{\zeta}^{N}(I+A(\hat{q}, k))^{-1} \Lambda_{\zeta}^{-N} g=(I+\tilde{A}(\hat{q}, \zeta, k))^{-1} g
$$

converges in $C^{\alpha}\left(\mathbf{R}^{3}\right)$ to a function which is $C^{\alpha}$ in $k$ when $\|\hat{q}\|_{\alpha, N}<\varepsilon_{0}$.

Now, given $\hat{q} \in H_{\alpha, N}$, we choose $\hat{q}_{\infty} \in C_{0}^{\infty}\left(\mathbf{R}^{3}\right)$ so that $\hat{q}_{1}=\hat{q}-\hat{q}_{\infty}$ satisfies $\left\|\hat{q}_{1}\right\|_{\alpha, N}<\varepsilon_{0}$. Thus we have Eq. (3.16) for $\tilde{g}=\tilde{h}+\tilde{A}\left(\hat{q}_{1}, \zeta, k\right) \tilde{h}+\tilde{q}_{\zeta}$. Our next objective is to show that

$$
\sup _{\zeta, k}\|\tilde{g}(\cdot, \zeta+v, k+s)-\tilde{g}(\cdot, \zeta, k)\|_{\alpha} \leqq C\left(|v|^{\alpha}+s^{\alpha}\right)
$$

for $|v|<1,0<s<1$.

Since

$$
\left(I+\tilde{A}\left(\hat{q}_{\infty}, \zeta, k\right)\left(I+\tilde{A}\left(\hat{q}_{1}, \zeta, k\right)\right)^{-1}\right)^{-1}=\left(I+\tilde{A}\left(\hat{q}_{1}, \zeta, k\right)\right)(I+\tilde{A}(\hat{q}, \zeta, k))^{-1},
$$

it follows from the uniform boundedness of $(I+\tilde{A}(\hat{q}, \zeta, k))^{-1}$ and Theorem 2.1 applied to $\tilde{A}\left(\hat{q}_{1}, \zeta, k\right)$ that

$$
\sup _{\zeta, k}\left\|\left(I+\tilde{A}\left(\hat{q}_{\infty}, \zeta, k\right)\left(I+\tilde{A}\left(\hat{q}_{1}, \zeta, k\right)\right)^{-1}\right)^{-1}\right\|_{\alpha}<\infty .
$$

Thus (3.16) shows that $\sup \|\tilde{g}(\cdot, \zeta, k)\|_{\alpha}<\infty$. Applying the difference operator in $k, \Delta(s)$, to (3.16) we have

$$
\begin{aligned}
& \Delta(s) \tilde{g}+\tilde{A}\left(\hat{q}_{\infty}, \zeta, k\right)\left(I+\tilde{A}\left(\hat{q}_{1}, \zeta, k\right)\right)^{-1} \Delta(s) \tilde{g} \\
& \quad=-\left[\Delta(s)\left(\tilde{A}\left(\hat{q}_{\infty}, \zeta, k\right)\left(I+\tilde{A}\left(\hat{q}_{1}, \zeta, k\right)\right)^{-1}\right]\left(\tilde{g}(\cdot, \zeta, k+s)-\tilde{q}_{\zeta}\right) \equiv r(\zeta, \zeta, k, s) .\right.
\end{aligned}
$$

Viewing (3.20) as a linear equation for $\Delta(s) \tilde{g}$, we need to show that the inhomogeneous term in this equation, $r(\xi)$, is bounded in $C^{\alpha}\left(\mathbf{R}^{3}\right)$ by a multiple of $s^{\alpha}$ uniformly in $(\zeta, k)$. To do this we will substitute the Neumann series for $\left(I+\tilde{A}\left(\hat{q}_{1}, \zeta, k\right)\right)^{-1}$ into (3.20) and consider $\partial_{\xi}^{\beta} r$. The terms in the resulting expansion for $\partial_{\xi}^{\beta} r$ are precisely those in (3.18) with $\hat{q}\left(\xi-\eta_{1}\right)$ in each $Q_{p}$ replaced by $\partial_{\xi}^{\beta}\left(\Lambda_{\zeta}^{N}(\xi) \hat{q}_{\infty}\left(\xi-\eta_{1}\right)\right)$, all other $\hat{q}$ 's in $Q_{p}$ and $R_{p}$ replaced by $\hat{q}_{1}$ 's and $f\left(\eta_{n}\right)=\Lambda_{\zeta}^{-N}(\eta)$ $\left(\tilde{g}\left(\eta_{n}, \zeta, k+\zeta\right)-q_{\zeta}\left(\eta_{n}\right)\right)$. Thus (3.19) implies

$$
\sup _{(\xi, \zeta, k)}\left|\partial_{\xi}^{\beta} r(\xi, \zeta, k, s)\right| \leqq C s^{\alpha} .
$$

Hence, using $\sup _{\xi}\left(|r(\xi)|+\sum_{|\beta|=1}\left|\partial_{\xi}^{\beta} r(\xi)\right|\right)$ to bound $\|r(\cdot, \zeta, k, s)\|_{\alpha}$, we conclude

$$
\sup _{\zeta, k}\|\tilde{g}(\cdot, \zeta, k+s)-\tilde{g}(\cdot, \zeta, k)\|_{\alpha} \leqq C s^{\alpha}
$$

for $0 \leqq s \leqq 1$.

To get the analogous result in $\zeta$ we let $\Delta(v) f=f(\zeta+v)-f(\zeta)$ for functions depending on $\zeta$. The analogue of (3.20) is 


$$
\begin{aligned}
\Delta(v) \tilde{g} & +\tilde{A}\left(\hat{q}_{\infty}, \zeta, k\right)\left(I+\tilde{A}\left(\hat{q}_{1}, \zeta, k\right)\right)^{-1} \Delta(v) \tilde{g} \\
= & -\left[\Delta(v)\left(\tilde{A}\left(\hat{q}_{\infty}, \zeta, k\right)\left(I+\tilde{A}\left(\hat{q}_{1}, \zeta, k\right)\right)^{-1}\right)\right]\left(\tilde{g}(\cdot, \zeta+v, k)-\tilde{q}_{\zeta+v}\right) \\
& -\tilde{A}\left(\hat{q}_{\infty}, \zeta, k\right)\left(I+\tilde{A}\left(\hat{q}_{1}, \zeta, k\right)\right)^{-1}\left(\Delta(v) \tilde{q}_{\zeta}\right) \equiv r_{1}+r_{2} .
\end{aligned}
$$

Since

$$
\tilde{A}\left(\hat{q}_{\infty}, \zeta, k\right)\left(I+\tilde{A}\left(\hat{q}_{1}, \zeta, k\right)^{-1}=\Lambda_{\zeta}^{N} A\left(\hat{q}_{\infty}, k\right)\left(I+A\left(\hat{q}_{1}, k\right)\right)^{-1} \Lambda_{\zeta}^{-N},\right.
$$

it follows directly from (2.5) and our bound on $\|\tilde{g}(\cdot, \zeta, k)\|_{\alpha}$ that

$$
\sup _{\zeta, k}\left\|r_{1}(\cdot, \zeta, k, v)\right\|_{\alpha} \leqq C|v| .
$$

To estimate $r_{2}$ we again substitute the Neumann series for $\left(I+\tilde{A}\left(\hat{q}_{1}, \zeta, k\right)\right)^{-1}$ and consider $\partial_{\xi}^{\beta} r_{2}$. The $n^{\text {th }}$ term in the resulting series for $\partial_{\xi}^{\beta} r_{2}$ is

$$
\begin{aligned}
& I_{n}(\xi, \zeta, k, v)=\int_{\mathbf{R}^{3 n}} \Lambda_{\zeta}^{-N}(\xi) \\
& . \frac{\partial_{\xi}^{\beta}\left(\Lambda_{\zeta}^{N}(\xi) \hat{q}_{\infty}\left(\xi-\eta_{1}\right)\right) \hat{q}_{1}\left(\eta_{1}-\eta_{2}\right) \cdots \hat{q}_{1}\left(\eta_{n-1}-\eta_{n}\right)\left(\Lambda_{\zeta}^{-N}\left(\eta_{n}\right)\left(\Lambda_{\zeta}^{N}(\xi) \Delta(v) \hat{q}_{\zeta}\left(\eta_{k}\right)\right)\right.}{(2 \pi)^{3 n} \prod_{j=1}^{n}\left(\left|\eta_{j}\right|^{2}-(k+i 0)^{2}\right)}
\end{aligned}
$$

$\cdot d \eta_{1} \cdots d \eta_{n}$.

We have

$$
\begin{aligned}
\Lambda_{\zeta}^{-N}\left(\eta_{n}\right)\left(\Delta(v) \tilde{q}_{\zeta}\left(\eta_{n}\right)\right)= & \hat{q}\left(\eta_{n}-\zeta-v\right)-\hat{q}(\eta-\zeta) \\
& +\Lambda_{\zeta}^{-N}\left(\eta_{n}\right)\left(\Lambda_{\zeta+v}^{N}\left(\eta_{n}\right)-\Lambda_{\zeta}^{N}\left(\eta_{n}\right)\right) \hat{q}\left(\eta_{n}-\zeta-v\right) .
\end{aligned}
$$

If we think of $\zeta$ as the variable for which we expect functions to be $C^{\alpha}$ and $\xi$ as a parameter (note that $\Lambda_{\zeta}(\xi)=\Lambda_{\xi}(\zeta)$ ), Theorem 2.1 and (2.5) imply

$\left|I_{n}(\xi, \zeta, k, v)\right| \leqq C|v|^{\alpha} \widetilde{C}^{n}\left\|\Lambda^{N} \hat{q}\right\|_{\alpha}\left\|\Lambda^{N} \hat{q}_{1}\right\|_{\alpha}^{n-1}\left(\left\|\Lambda_{\xi}^{N}(\cdot) \Lambda_{\zeta}^{-N}(\xi) \partial_{\xi}^{\beta}\left(\Lambda_{\zeta}^{N}(\xi) \hat{q}_{\infty}(\xi-\cdot)\right)\right\|\right)_{\alpha}$.

Thus

$$
\sup _{(\xi, \zeta, k)}\left|\partial_{\xi}^{\beta} r_{2}(\xi, \zeta, k, v)\right| \leqq C|v|^{\alpha}
$$

and, using this to bound $\left\|r_{2}(\cdot, \zeta, k, v)\right\|_{\alpha}$ as before, we conclude

$$
\sup _{\zeta, k}\|\tilde{g}(\cdot, \zeta+v, k)-\tilde{g}(\cdot, \zeta, k)\|_{\alpha} \leqq C|v|^{\alpha}
$$

for $|v| \leqq 1$.

Now we are ready to go back to $\tilde{h}$ via the relation

$$
\tilde{h}=\left(I+\tilde{A}\left(\hat{q}_{1}, \zeta, k\right)\right)^{-1} \tilde{g}-\left(I+\tilde{A}\left(\hat{q}_{1}, \zeta, k\right)\right)^{-1} \tilde{q}_{\zeta} \equiv \tilde{h}_{1}-\tilde{h}_{2} .
$$

That the $C^{\alpha}$-norm in $(\xi, \zeta, k),\left\|\tilde{h}_{2}\right\|_{\alpha}$, is finite follows by substitution of the Neumann series exactly as in the derivation of (3.21) and (3.23). That $\left\|\tilde{h}_{1}\right\|_{\alpha}<\infty$ also follows by substitution of the Neumann series but first we separate terms:

$$
\Delta(\rho)(I+\tilde{A})^{-1} \tilde{g}=\left(\Delta(\rho)(I+\tilde{A})^{-1}\right) \tilde{\tilde{g}}+(I+\tilde{A})^{-1} \Delta(\rho) \tilde{g},
$$

where $\rho=s$ or $v$ and $\tilde{g}$ is $\tilde{g}$ with $\rho$ added to the appropriate variable. The first term on the right of (3.24) is estimated by (3.19) with $f=\tilde{\tilde{g}}$ when $\rho=s$ and is 
estimated trivially when $\rho=v$. The second term can be estimated directly by $\left(3.14^{\prime}\right)$ since $\|\Delta(\rho) \tilde{g}(\cdot, \zeta, k)\|_{\alpha} \leqq C|\rho|^{\alpha}$.

From the proof of Theorem 3.2 one can see that the mapping

$$
\Psi: \hat{q} \rightarrow \tilde{h}
$$

is analytic from $\mathcal{O}$ to $C^{\alpha}\left(\mathbf{R}^{3} \times \mathbf{R}^{3} \times \overline{\mathbf{R}}_{+}\right)$. To do this, given $\hat{q}_{0} \in \mathcal{O}$, we consider for $\|\hat{q}\|_{\alpha, N} \leqq 1$ and $|z| \leqq \delta$,

$$
\tilde{h}(\xi, \zeta, k, z)=-\left(I+\tilde{A}\left(\hat{q}_{0}+z \hat{q}, \zeta, k\right)\right)^{-1}\left(\tilde{q}_{0}+z \tilde{q}\right)_{\zeta} .
$$

Writing

$$
\begin{aligned}
\left(I+\tilde{A}\left(\hat{q}_{0}+z \hat{q}, \zeta, k\right)\right)^{-1}\left(\tilde{q}_{0}+z \tilde{q}\right)_{\zeta} & =\left(I+z\left(I+\widetilde{A}\left(\hat{q}_{0}, \zeta, k\right)\right)^{-1} \tilde{A}(\hat{q}, \zeta, k)\right)^{-1}\left(I+\tilde{A}\left(\hat{q}_{0}, \zeta, k\right)\right)^{-1}\left(\tilde{q}_{0}+z \tilde{q}\right)_{\zeta},
\end{aligned}
$$

Theorem 2.1 and $\left(3.14^{\prime}\right)$ imply that for $\delta$ sufficiently small we can expand the first factor on the right of (3.25) in a Neumann series which converges in $C^{\alpha}\left(\mathbf{R}^{3}\right)$ for all $(\zeta, k) \in \mathbf{R}^{3} \times \overline{\mathbf{R}}_{+}$. Thus for some $\delta$ independent of $(\xi, \zeta, k)$ we have for $\|\hat{q}\|_{\alpha, N} \leqq 1$ and $|z|<\delta$,

$$
\tilde{h}(\xi, \zeta, k, z)=\frac{1}{2 \pi i} \oint_{|w|=\delta} \frac{\tilde{h}(\zeta, \zeta, k, w)}{w-z} d w
$$

and hence for all $k \geqq 0$,

$$
\frac{\partial^{p} \tilde{h}}{\partial z^{p}}(\xi, \zeta, k, 0)=\frac{p !}{2 \pi i} \int_{|w|=\delta} \frac{\tilde{h}(\xi, \zeta, k, w) d w}{w^{p+1}} .
$$

Since $\Psi\left(\hat{q}_{0}+z \hat{q}\right)=\tilde{h}(\xi, \zeta, k, z)$, to conclude that $\Psi\left(\hat{q}_{0}+z \hat{q}\right)$ can be expanded for $|z|<\delta$ in a power series in $z$ convergent in $C^{\alpha}\left(\mathbf{R}^{3} \times \mathbf{R}^{3} \times \overline{\mathbf{R}}_{+}\right)$uniformly on $\|\hat{q}\|_{\alpha, N} \leqq 1$, we only need to show that

$$
\|\tilde{h}(\cdot, \cdot ;, w)\|_{\underline{\alpha}} \leqq C
$$

for $|w|=\delta$. However, this is just the statement that the estimate in Theorem 3.2 is locally uniform in $\hat{q}$. This uniformity is clear from the proof. Thus we have shown that $\Psi$ satisfies one of the definitions of analyticity (see Pöschel-Trubowitz [12], Appendix A, or Nachbin [11]) and have

Corollary 3.4. The mapping $\Psi: \hat{q} \rightarrow \tilde{h}$ considered as a function from $\mathcal{O}$ to $C^{\alpha}\left(\mathbf{R}^{3} \times\right.$ $\mathbf{R}^{3} \times \overline{\mathbf{R}}_{+}$) is analytic in $\hat{q}$.

Analyticity in the sense above is equivalent to the fact that $\Psi$ has a continuous Frechet derivative with respect to $\hat{q}$ (see references above), as one can easily verify. In what follows we will often make use of the continuous differentiability of $\Psi$. If we restrict to the backscattering map on $\mathcal{O}$

$$
S: \hat{q} \rightarrow h(\xi,-\xi,|\xi|)=\Lambda_{-\xi}^{-N}(\xi) \tilde{h}(\xi,-\xi,|\xi|),
$$

Theorem 3.2 implies $\|S(\hat{q})\|_{\alpha, N}<\infty$. Moreover, choosing $\hat{q}_{n} \in \mathcal{O} \cap C_{0}^{\infty}\left(\mathbf{R}^{3}\right)$ converging to $\hat{q}$ in \|\|$_{\alpha, N}$, it follows from the analyticity and hence continuity of $\Psi$ that $\left\|\Psi\left(\hat{q}_{n}\right)-\Psi(\hat{q})\right\|_{\underline{\alpha}} \rightarrow 0$. Thus $\left\|S\left(\hat{q}_{n}\right)-S(\hat{q})\right\|_{\alpha, N} \rightarrow 0$. Since $\left\|S\left(\hat{q}_{n}\right)\right\|_{\alpha^{\prime} N^{\prime}}<\infty$ for 
all $N^{\prime}>1,0<\alpha^{\prime}<1$, it follows by Lemma 1.1 that $S\left(\hat{q}_{n}\right) \in H_{\alpha, N}$ and hence $S(\hat{q}) \in H_{\alpha, N}$. This gives:

Corollary 3.5. The backscattering map $S: \hat{q} \rightarrow h(\xi,-\xi,|\xi|)$ is an analytic function from 10 to $H_{\alpha, N}$.

\section{Section 4. The Derivative of the Backscattering Map}

Since by Corollary $3.4 \Psi: \hat{q} \rightarrow \tilde{h}$ is a continuously Frechet differentiable function on $\mathcal{O}$, we may compute its derivative. To do this we will differentiate Eq. (3.3) with respect to $\hat{q}$. Note that Theorem 2.1 implies $\tilde{A}(\hat{q}) \tilde{h}(\hat{q})$ is the composition of a bounded operator valued function linear in $\hat{q}$ with a continuously differentiable function, and is hence continuously differentiable. We have for $v \in H^{\alpha, N}, \hat{q} \in \mathcal{O}$,

and hence

$$
d \tilde{h}(v)+\tilde{A}(\hat{q}) d \tilde{h}(v)=-\tilde{v}_{\zeta}-\tilde{A}(v) \tilde{h}
$$

$$
d \tilde{h}(v)=(I+\tilde{A}(\hat{q}))^{-1}\left(-\tilde{v}_{\zeta}-\tilde{A}(v) \tilde{h}\right) .
$$

Lemma 4.1. The operator $(I+\tilde{A}(\hat{q}))^{-1}, \hat{q} \in \mathcal{O}$, has the following form:

$$
\left[(I+\tilde{A}(\hat{q}, \zeta, k))^{-1} f\right](\xi)=f(\xi)+(2 \pi)^{-3} \int \frac{\Lambda_{\zeta}^{N}(\xi) h(\xi, \eta, k) \Lambda_{\zeta}^{-N}(\eta) f(\eta)}{|\eta|^{2}-(k+i 0)^{2}} d \eta
$$

Proof. Let $f+\tilde{D} f$ denote the right-hand side of (4.2). Then we have from (3.3)

$$
(I+\tilde{A}(\hat{q}))(I+\tilde{D}) f=f+\tilde{A}(\hat{q}) f+\tilde{D} f+\tilde{A}(\hat{q}) \tilde{D} f=f+\tilde{A}(\tilde{q}) f+\tilde{D} f-\tilde{D} f-\tilde{A}(q) f=f .
$$

Thus $I+\tilde{D}$ is a right inverse for $I+\widetilde{A}(\hat{q})$. Since $I+\widetilde{A}(\hat{q})$ is invertible, it follows that $I+\tilde{D}=(I+\widetilde{A}(\hat{q}))^{-1}$.

Substituting (4.2) into (4.1) we have

$$
\begin{aligned}
d \tilde{h}(v)= & -\tilde{v}_{\zeta}-(2 \pi)^{-3} \int_{\mathbf{R}^{3}} \frac{\Lambda_{\zeta}^{N}(\xi) h(\xi, \eta, k) v_{\zeta}(\eta) d \eta}{|\eta|^{2}-(k+i 0)^{2}} \\
& -(2 \pi)^{-3} \int_{\mathbf{R}^{3}} \frac{h(\eta, \zeta, k) \Lambda_{\zeta}^{N}(\xi) v(\xi-\eta) d \eta}{|\eta|^{2}-(k+i 0)^{2}} \\
& -(2 \pi)^{-6} \int_{\mathbf{R}^{3}} \int_{\mathbf{R}^{3}} \frac{\Lambda_{\zeta}^{N}(\xi) h(\xi, t, k) v(t-\eta) h(\eta, \zeta, k) d \eta d t}{\left(|t|^{2}-(k+i 0)^{2}\right)\left(|\eta|^{2}-(k+i 0)^{2}\right)} .
\end{aligned}
$$

Changing variables so as to get integrals of $v(\eta)$ in all integrals in (4.3) and cancelling $\Lambda_{\zeta}^{N}(\xi)$ in all terms, one arrives at

$$
\begin{aligned}
d h(\xi, \zeta, k)(v)= & -v(\xi-\zeta)-(2 \pi)^{-3} \int_{\mathbf{R}^{3}} \frac{h(\xi, \eta+\zeta, k) v(\eta)}{|\eta+\zeta|^{2}-(k+i 0)^{2}} d \eta \\
& -(2 \pi)^{-3} \int_{\mathbf{R}^{3}} \frac{h(\xi-\eta, \zeta, k) v(\eta)}{|\eta-\xi|^{2}-(k+i 0)^{2}} d \eta \\
& -(2 \pi)^{-6} \int_{\mathbf{R}^{3}}\left(\int_{\mathbf{R}^{3}} \frac{h(\xi, t, k) h(t-\eta, \zeta, k) v(\eta) d \eta}{\left(|t|^{2}-(k+i 0)^{2}\right)\left(|t-\eta|^{2}-(k+i 0)^{2}\right)}\right) d t
\end{aligned}
$$


Since $I+\tilde{D}$ is the left inverse of $I+\tilde{A}(\hat{q})$, we have

$$
0=\hat{q}(\xi-\zeta)+h(\xi, \zeta, k)+(2 \pi)^{-3} \int_{\mathbf{R}^{3}} \frac{h(\xi, \eta, k) \hat{q}(\eta-\zeta)}{|\eta|^{2}-(k+i 0)^{2}} d \eta .
$$

Sending $\xi \rightarrow-\zeta, \zeta \rightarrow-\xi$ and $\eta \rightarrow-\eta$, we have

$$
0=\hat{q}(\xi-\zeta)+h(-\zeta,-\xi, k)+(2 \pi)^{-3} \int_{\mathbf{R}^{3}} \frac{\hat{q}(\xi-\eta) h(-\zeta,-\eta, k)}{|\eta|^{2}-(k+i 0)^{2}} d \eta .
$$

Comparing (4.5) and (3.1) one sees that for $\hat{q} \in \mathcal{O}$,

$$
h(-\zeta,-\xi, k)=h(\xi, \zeta, k)
$$

for $(\xi, \zeta, k) \in \mathbf{R}^{3} \times \mathbf{R}^{3} \times \overline{\mathbf{R}}_{+}$. Hence, setting $(\xi, \zeta, k)=(\xi,-\xi,|\xi|)$ in (4.4), sending $\eta \rightarrow 2 \eta$ and using (4.6), we have

$$
\begin{aligned}
d h(\xi,-\xi,|\xi|)(v)= & -v(2 \xi)-2 \pi^{-3} \int_{\mathbf{R}^{3}} \frac{h(\xi-2 \eta,-\xi,|\xi|) v(2 \eta)}{|\xi-2 \eta|^{2}-(|\xi|+i 0)^{2}} d \eta \\
& -2^{-3} \pi^{-6} \int_{\mathbf{R}^{3}}\left(\int_{\mathbf{R}^{3}} \frac{h(\xi, t,|\xi|) h(t-2 \eta,-\xi,|\xi|) v(2 \eta) d \eta}{\left(|t|^{2}-(|\xi|+i 0)^{2}\right)\left(|2 \eta-t|^{2}-(|\xi|+i 0)^{2}\right)}\right) d t .
\end{aligned}
$$

From (4.7) one sees that the Frechet derivative of the backscattering map $S$ is given by

$$
\begin{aligned}
d S & =(I+B+F) T, \\
{[T f](\xi) } & =-f(2 \xi),[B f](\xi)=2 \pi^{-3} \int_{\mathbf{R}^{3}} \frac{h(\xi-2 \eta,-\xi,|\xi|) f(\eta)}{|\xi-2 \eta|^{2}-(|\xi|+i 0)^{2}} d \eta
\end{aligned}
$$

and

$$
[F f](\xi)=2^{-3} \pi^{-6} \int_{\mathbf{R}^{3}}\left(\int_{\mathbf{R}^{3}} \frac{h(\xi, t,|\xi|) h(t-2 \eta,-\xi,|\xi|) f(\eta) d \eta}{\left(|t|^{2}-(|\xi|+i 0)^{2}\right)\left(|2 \eta-t|^{2}-(|\xi|+i 0)^{2}\right)}\right) d t .
$$

Since $S$ is an analytic function, $d S$ is continuous on $\mathcal{O}$ as a function with values in $\mathscr{L}\left(H_{\alpha, N}\right)$, the space of bounded linear operators from $H_{\alpha, N}$ to itself. Since we need to know that $B$ and $F$ are individually continuous functions from $\mathcal{O}$ to $\mathscr{L}\left(H_{\alpha, N}\right)$, we prove the following.

Lemma 4.2. $B(\hat{q})$ is an analytic function from $\mathcal{O}$ to $\mathscr{L}\left(H_{\alpha, N}\right)$.

Proof. As in the proof of Corollary 3.4, the analyticity will follow from the local boundedness of the operator norm $\left\||B(\hat{q}) \||_{\alpha, N}\right.$ on $\mathcal{O}$.

From (3.17) we have the representation

$$
h(\xi-2 \eta,-\xi,|\xi|)=\left[\left(I+A\left(\hat{q}_{1},|\xi|\right)\right)^{-1}\left(\Lambda_{-\xi}^{-N}(\cdot) g(\cdot,-\xi,|\xi|)-\hat{q}(\cdot+\xi)\right)\right](\xi-2 \eta) .
$$

As in the proof of Theorem 3.2 we will substitute the Neumann series for $\left(I+A\left(\hat{q}_{1},|\xi|\right)\right)^{-1}$ in $h$ and hence in $B(\hat{q})$. This gives

$$
[B(\hat{q}) f](\xi)=2 \pi^{-3} \sum_{n=0}^{\infty}\left(\frac{-1}{(2 \pi)^{3}}\right)^{n} I_{n}(\xi),
$$


where

$$
\begin{aligned}
I_{n}(\xi)= & \int_{\mathbf{R}^{3(n+1)}} \\
& \frac{f(\eta) \hat{q}_{1}\left(\xi-2 \eta-\eta_{1}\right) \hat{q}_{1}\left(\eta_{1}-\eta_{2}\right) \cdots \hat{q}_{1}\left(\eta_{n-1}-\eta_{n}\right)\left(\Lambda_{-\xi}^{-N}\left(\eta_{n}\right) g\left(\eta_{n},-\xi,|\xi|\right)-\hat{q}\left(\eta_{n}+\xi\right)\right)}{\left(|\xi-2 \eta|^{2}-(|\xi|+i 0)^{2}\right) \prod_{i=1}^{n}\left(\left|\eta_{i}\right|^{2}-(|\xi|+i 0)^{2}\right)}
\end{aligned}
$$

$\cdot d \eta d \eta_{1} \cdots d \eta_{n}$.

Setting $\eta_{0}=\xi-2 \eta$, we have

$$
\begin{aligned}
I_{n}(\xi) & =\frac{1}{8} \int_{\mathbf{R}^{3(n+1)}} \\
& \frac{f\left(\frac{\xi-\eta_{0}}{2}\right) \hat{q}_{1}\left(\eta_{0}-\eta_{1}\right) \hat{q}_{1}\left(\eta_{1}-\eta_{2}\right) \cdots \hat{q}_{1}\left(\eta_{n-1}-\eta_{n}\right)\left(\Lambda_{-\xi}^{-N}\left(\eta_{n}\right) g\left(\eta_{n},-\xi,|\xi|\right)-\hat{q}\left(\eta_{n}+\xi\right)\right)}{\prod_{i=0}^{n}\left(\left|\eta_{i}\right|^{2}-(|\xi|+i 0)^{2}\right)} \\
& \cdot d \eta_{0} \cdots d \eta_{n} .
\end{aligned}
$$

These are precisely the terms which arose at the end of the proof of Theorem 3.2 with one factor of $\hat{q}$, replaced by $f, \zeta=-\xi$ and $k=|\xi|$. Hence, the argument given there shows that

$$
\left\|\Lambda^{N} B(\hat{q}) f\right\|_{\alpha} \leqq C\|f\|_{\alpha, N}
$$

for $f \in H_{\alpha, N}$, where $C$ is locally uniform in $\hat{q}$ on $\mathcal{O}$.

To prove analyticity we proceed as follows. Defining $h(\eta, \zeta, k, z)$ as in the proof of Corollary 3.4, we have

$$
\left[B\left(\hat{q}_{0}+z \hat{q}\right) f\right](\xi)=2 \pi^{-3} \int_{\mathbf{R}^{3}} \frac{f\left(\frac{\xi-\eta}{2}\right) h(\eta,-\xi,|\xi|, z)}{|\eta|^{2}-\left(|\xi|^{2}+i 0\right)^{2}} d \eta,
$$

and we know that $\Lambda_{-\xi}^{N}(\eta) h(\eta,-\xi,|\xi|, z)$ is an analytic function from $|z| \leqq \delta$ to $C^{\alpha}\left(\mathbf{R}^{3}\right)$ for each $\xi \in \mathbf{R}^{3}$ and $\hat{q}$ with $\|\hat{q}\|_{\alpha, N} \leqq 1$. Thus Theorem 2.1 implies that for each $\xi \in \mathbf{R}^{3}$ and $f \in H_{\alpha, N}$ we can represent $\left[B\left(\hat{q}_{0}+z \hat{q}\right) f\right](\xi)$ as a Cauchy integral over $|z|=\delta / 2$ with $\delta$ independent of $\xi$ and $\hat{q}$, when $\|\hat{q}\|_{\alpha, N} \leqq 1$. Now analyticity follows from (4.8) just as in the proof of Corollary 3.4.

To see that the range of $B(\hat{q})$ on $H_{\alpha, N}$ is contained in $H_{\alpha, N}$, we approximate $\hat{q}$ by $\hat{q}_{n} \in C_{0}^{\infty}\left(\mathbf{R}^{3}\right)$ as in the proof of Corollary 3.5 .

The main result of this section is that, for $\hat{q} \in \mathcal{O}$, the operator $d S(\hat{q})$ is Fredholm of index zero on $H_{\alpha, N}$. To prove this we will show that $B^{2}$ and $F$ are compact on $H_{\alpha, N}$. To see that this is sufficient, note that for $0 \leqq \varepsilon \leqq 1$,

$$
\begin{aligned}
T^{-1}(1-\varepsilon B)(I+\varepsilon B+F) T & =I+K_{1}, \\
\left.(I+\varepsilon B+F) T^{-1}(I-\varepsilon B)\right) & =I+K_{2},
\end{aligned}
$$

where $K_{1}$ and $K_{2}$ are compact if $B^{2}$ and $F$ are. Hence, $(I+\varepsilon B+F) T$ is Fredholm 
for $0 \leqq \varepsilon \leqq 1$, and for $\varepsilon=0$ it is a compact perturbation of an invertible operator, and hence of index zero. Thus, to conclude that $d S(\hat{q})$ is a Fredholm operator of index zero on $H_{\alpha, N}$ for $\hat{q} \in \mathcal{O}$, we only need the following:

Theorem 4.3. The operators $B^{2}(\hat{q})$ and $F(\hat{q})$ are compact on $H_{\alpha, N}$ for $\hat{q} \in \mathcal{O}$.

To prove Theorem 4.3 we will first take advantage of the fact that operator norm limits of compact operators are compact to replace $B^{2}$ and $F$ by the operators:

$$
\left[T_{1} f\right](\xi)=\int_{\mathbf{R}^{6}} \frac{g_{1}(\xi, \eta, t) f(t)}{\left(|\xi-2 \eta|^{2}-(|\xi|+i 0)^{2}\right)\left(|\eta-2 t|^{2}-(|\eta|+i 0)^{2}\right)} d \eta d t
$$

and

$$
\left[T_{2} f\right](\xi)=\int_{\mathbf{R}^{6}} \frac{g_{2}(\xi, \eta, t) f(t)}{\left.|\eta|^{2}-(|\xi|+i 0)^{2}\right)\left(|\eta-2 t|^{2}-(|\xi|+i 0)^{2}\right)} d \eta d t,
$$

respectively, where $g_{i}, i=1,2$, satisfies

(i) $g_{i} \in C^{\infty}\left(\mathbf{R}^{9}\right)$ and all of its partial derivatives are bounded,

(ii) $g_{i}(\xi, \eta, t)=0$ for $|\xi|+|\eta|+|t|<\delta$ for some $\delta>0$, and

(iii) $g_{i}(\xi, \eta, t)=0$, if $|\xi-\eta|>M$ or $|\eta-t|>M$ for some $M<\infty$.

Then the proof proceeds by analysis of the singularities of the kernels $t_{1}(\xi, t)$ and $t_{2}(\xi, t)$ of $T_{1}$ and $T_{2}$. For this we will use estimates modelled on the following simple lemma.

Lemma 4.4. Assume that $g(\xi, \eta)$ is supported in $|\eta-\eta(\xi)|<M$ and that $\|g\|_{\alpha}<\infty$ for some $\alpha \in(0,1)$. Assume that $h(\xi)$ satisfies $|h(\xi+\mu)-h(\xi)| \leqq C|\mu|$ for $|\mu| \leqq 1, \xi \in \mathbf{R}^{n}$. Let

Then

$$
s(\xi)=\int_{\mathbf{R}^{n}} \frac{g(\xi, \eta)}{\eta_{1}-h(\xi)-i 0} d \eta
$$

$$
\|s\|_{\alpha^{\prime}}<C\left(M,\|g\|_{\alpha},\|h\|_{1}, \alpha^{\prime}\right)
$$

for any $\alpha^{\prime}<\alpha$.

Proof. Changing variables we have

$$
s(\xi)=\int_{\mathbf{R}^{n}} \frac{g\left(\xi, \eta+h(\xi) \hat{e}_{1}\right)}{\eta_{1}-i 0} d \eta .
$$

Letting $f(\xi, \eta)=g\left(\xi, \eta+h(\xi) \hat{e}_{1}\right)$, we see $f$ satisfies the same hypotheses $g$ did. Expanding (4.10) we have, letting $\eta=\left(\eta_{1}, \eta^{\prime}\right)$,

$s(\xi)=\int_{\left|\eta_{1}\right|>1} \frac{f(\xi, \eta)}{\eta_{1}} d \eta+\int_{\left|\eta_{1}\right|<1} \frac{f(\xi, \eta)-f\left(\xi, 0, \eta^{\prime}\right)}{\eta_{1}} d \eta+\int_{\left|\eta_{1}\right|<1} \frac{f\left(\xi, 0, \eta^{\prime}\right)}{\eta_{1}-i 0} d \eta=I_{1}+I_{2}+I_{3}$.

Carrying out the integration in $\eta_{1}$ in $I_{3}$,

$$
I_{3}=\pi i \int_{\mathbf{R}^{n-1}} f\left(\xi, 0, \eta^{\prime}\right) d \eta^{\prime} .
$$

Since $\left\|f(\cdot, \eta)-f\left(\cdot, 0, \eta^{\prime}\right)\right\|_{\alpha^{\prime}} \leqq 3\left|\eta_{1}\right|^{\alpha-\alpha^{\prime}}\|f\|_{\alpha}$, the $\alpha^{\prime}$-norm of $I_{2}$ is easily estimated, and (4.11) follows directly from the representation of $s(\xi)$ as $I_{1}+I_{2}+I_{3}$. 
The problem of obtaining (4.11) for singular integrals with more general denominators can be reduced to Lemma 4.4 by change of variables as long as the gradient in $\eta$ of the denominator is bounded away from zero near the surface where the denominator vanishes. In what follows we will leave such reductions to the reader and simply refer to Lemma 4.4.

Proof of Theorem 4.3. Lemma 4.2 shows that $B(\hat{q})$ is analytic in $\hat{q}$, and hence, since $d S(\hat{q})$ is analytic, $F(\hat{q})$ is also an analytic function of $\hat{q}$. Thus, making a change of arbitrarily small norm in $B^{2}(\hat{q})$ and $F(\hat{q})$, we may assume $\hat{q} \in C_{0}^{\infty}\left(\mathbf{R}^{3}\right) \cap \mathcal{O}$, and hence by Theorem 3.2

$$
\left\|\Lambda_{\zeta}^{N^{\prime}} h\right\|_{\underline{\alpha}^{\prime}}<\infty
$$

for all $N^{\prime}>1$ and $\alpha^{\prime}<1$.

The operators $B^{2}(\hat{q})$ and $F(\hat{q})$ are given by

$$
\left[B^{2} f\right](\xi)=4 \pi^{-6} \int_{\mathbf{R}^{6}} \frac{h(\xi-2 \eta,-\xi,|\xi|) h(\eta-2 t,-\eta,|\eta|) f(t) d t d \eta}{\left(|\xi-2 \eta|^{2}-(|\xi|+i 0)^{2}\right)\left(|\eta-2 t|^{2}-(|\eta|+i 0)^{2}\right)}
$$

and

$$
[F f](\xi)=2^{-3} \pi^{-6} \int_{\mathbf{R}^{6}} \frac{h(-\eta,-\xi,|\xi|) h(\eta-2 t,-\xi,|\xi|) f(t)}{\left(|\eta|^{2}-(|\xi|+i 0)^{2}\right)\left(|\eta-2 t|^{2}-(|\xi|+i 0)^{2}\right)} d t d \eta .
$$

By the argument used in the proof of Lemma 1.1, (4.12) implies that, given $\alpha_{1}, \alpha<\alpha_{1}<1$, we can choose $h_{n}(\xi, \zeta) \in C^{\infty}\left(\mathbf{R}^{6}\right)$ such that $h_{n}(\xi, \zeta)=0$ for $|\xi+\zeta|$ sufficiently large, $\partial_{\xi, \zeta}^{\beta} h_{n}$ is bounded for all $\beta$ and

$$
\Lambda^{N}(\xi+\zeta)\left(h_{n}(\xi, \zeta)-h(\xi,-\zeta,|\zeta|)\right)
$$

tends to zero in $C^{\alpha_{1}}\left(\mathbf{R}^{6}\right)$. Replacing the $h$ 's in $B^{2}$ and $F$ by $h_{n}$ 's with the appropriate arguments, we get $B_{n}^{2}$ and $F_{n}$. We claim that $\left\|\left|B_{n}^{2}-B^{2} \|\right|_{\alpha, N}\right.$ and $\|\left|F_{n}-F \|\right|_{\alpha, N}$ go to zero as $n \rightarrow \infty$. Expanding $B_{n}^{2}-B^{2}=B_{n}\left(B_{n}-B\right)+\left(B_{n}-B\right) B$ and making the analogous expansion of $F_{n}-F$, one sees by the estimates on $\left|I_{1}\right|,\left|I_{2}\right|$ and $\left|I_{3}\right|$ in the proof Theorem 2.1, that $\left\|\left|B_{n}^{2}-B^{2} \|\right|_{0, N}\right.$ and $\|\left|F_{n}-F \|\right|_{0, N}$ go to zero. To estimate $\Delta(\mu)\left(\Lambda^{N}\left(B^{2}-B_{n}^{2}\right)\right)$ and $\Delta(\mu)\left(\Lambda^{N}\left(F_{n}-F\right)\right)$ we first change variables in $\eta$ and $t$ so that when $\xi$ appears in the denominator of an integrand it is in a factor of the form $\left(|\eta|^{2}-(|\xi|+i 0)^{2}\right)$ or $\left(|t|^{2}-(|\xi|+i 0)^{2}\right)$. Then $\left[\Delta(\mu)\left(\Lambda^{N}\left(B^{2}-B_{n}^{2}\right) f\right](\xi)\right.$ and $\left[\Delta(\mu)\left(\Lambda^{N}\left(F-F_{n}\right) f\right](\xi)\right.$ can be expanded into sums of terms where the difference operator acts on $\left(|\beta|^{2}-(|\xi|+i 0)^{2}\right)^{-1}, \beta=\eta$ or $t$, which we estimate by Theorem 2.2; terms where the operator acts on $f(l(\xi, \eta, t)), l$ a linear function, which we estimate by Theorem 2.1 with $f$ playing the role of $\hat{q}$, and terms where the operator acts on $\Lambda^{N}, h, h_{n}$ or $h-h_{n}$, which we again expand as $I_{1}+I_{2}+I_{3}$ and then estimate $\left|I_{1}\right|,\left|I_{2}\right|$ and $\left|I_{3}\right|$ as the proof of Theorem 2.1. It is estimating terms of the last type that we use $\alpha_{1}>\alpha$ and this makes all estimates substantially easier. Thus, making a change of arbitrarily small operator norm, we can replace $B^{2}$ and $F$ by operators $T_{1}$ and $T_{2}$ as in (4.9) with $g_{1}$ and $g_{2}$ satisfying (i) and (iii).

To see that we can make the integrands in $B_{n}^{2}$ and $F_{n}$ vanish for $|\xi|+|\eta|+|t|<\delta_{0}$ so that $g_{1}$ and $g_{2}$ will satisfy (ii) in (4.9), we proceed as follows. Given any $\varphi \in C_{0}^{\infty}(|x|<1)$ and $\delta>0$, let 


$$
\begin{aligned}
{\left[R_{n} f\right](\delta \xi) } & =\int_{\mathbf{R}^{6}} \frac{h_{n}(\delta \xi-2 \eta, \delta \xi) \varphi^{2}\left(\frac{\eta}{\delta}\right) h_{n}(\eta-2 t, \eta) \varphi^{2}\left(\frac{t}{\delta}\right) f(t)}{\left(|\delta \xi-2 \eta|^{2}-(|\delta \xi|+i 0)^{2}\right)\left(|\eta-2 t|^{2}-(|\eta|+i 0)^{2}\right)} d t d \eta \\
& =\delta^{2} \int_{\mathbf{R}^{6}} \frac{h_{n}(\delta \xi-2 \delta \eta, \delta \xi) \varphi^{2}(\eta) h_{n}(\delta \eta-2 \delta t, \delta \eta) \varphi^{2}(t) f(\delta t)}{\left(|\xi-2 \eta|^{2}-(|\xi|+i 0)^{2}\right)\left(|\eta-2 t|^{2}-(|\eta|+i 0)^{2}\right)} d t d \eta
\end{aligned}
$$

Thus, by Theorems 2.1 and 2.2,

$$
\left\|\varphi(\xi)\left[R_{n} f\right](\delta \xi)\right\|_{\alpha, N} \leqq C \delta^{2}\|\varphi(t) f(\delta t)\|_{\alpha, N} .
$$

Thus, since $\|g(\xi)\|_{\alpha} \leqq \delta^{-\alpha}\|g(\delta \xi)\|_{\alpha}$ for $\delta<1$, we see that $\left\|\left|\varphi(\xi / \delta) R_{n} \|\right|_{\alpha, N} \leqq C \delta\right.$. Thus, making an arbitrarily small norm change in $B_{n}^{2}$, we can assume that its integrand vanishes for $|\xi|+|\eta|+|t|<\delta_{0}$ for some $\delta_{0}>0$. This argument applies to $F_{n}$ as well. Thus we may replace $B_{n}^{2}$ and $F_{n}$ by the operators $T_{1}$ and $T_{2}$ in (4.9) with $g_{1}$ and $g_{2}$ satisfying (i), (ii) and (iii).

We will now study $T_{1}$. The analysis of $T_{2}$ is very similar and somewhat easier, and we will sketch it at the end of the proof.

In terms of $\eta$ the integral defining $T_{1}$ is singular on the sphere (if $\xi \neq 0$ )

$$
\sum=\{\eta:|\xi-2 \eta|=|\xi|\}
$$

and the plane (if $t \neq 0$ )

$$
\Pi=\left\{\eta:|t|^{2}-\eta \cdot t=0\right\}
$$

We will see that the kernel $t_{1}(\xi, t)$ of $T_{1}$ is most singular at points $(\xi, t)$ for which $\sum$ and $\prod$ are tangent. This happens when

$$
\left\{\eta: \eta=\frac{\xi}{2} \pm \frac{|\xi|}{2} \frac{t}{|t|}\right\} \cap\left\{\eta: \eta \cdot t-|t|^{2}=0\right\} \neq \phi
$$

i.e. when $\xi \cdot t \pm|\xi||t|-2|t|^{2}=0$. With these facts in mind we will break up the integration in $\eta$ by summing over a partition of unity generated by $\rho_{1}=$ $\rho(|\xi-2 \eta|-|\xi|)$ and $\rho_{2}=\rho\left(|t|-(\eta \cdot t)|t|^{-1}\right)$, where $\rho \in C_{0}^{\infty}(\mathbf{R})$ satisfies $\rho(s)=1$ for $|s|<\varepsilon_{1}$ and $\rho(s)=0$ for $|s|>2 \varepsilon_{1}$. Since $g_{1}=0$ for $|\xi|+|\eta|+|t|<\delta$, choosing $\varepsilon_{1}$ sufficiently small, we can assume that $|\xi|>\delta / 4$ on the support of $\rho_{1} \rho_{2} g_{1}$.

We will also need cutoffs in $t$ near the most singular set,

$$
\beta_{1}=\beta\left(\frac{2|t|-(\xi \cdot t)|t|^{-1}-|\xi|}{|\xi|}\right) \text { and } \beta_{2}=\beta\left(\frac{2|t|-(\xi \cdot t)|t|^{-1}+|\xi|}{|\xi|}\right) \text {, }
$$

where $\beta \in C_{0}^{\infty}(\mathbf{R})$ satisfies $\beta(s)=1$ for $|s|<\varepsilon_{2}$ and $\beta(s)=0$ for $|s|>2 \varepsilon_{2}$. The constants $\varepsilon_{1}$ and $\varepsilon_{2}$ are chosen small enough that on the support of $\rho_{1} \rho_{2} \beta_{i} g_{1}, i=1,2$, the component of $\xi-2 \eta$ orthogonal to $t$ has length less than $1 / 2|\xi|$. Note that on support $\rho_{1} \rho_{2} \beta_{i} g_{1},|\xi-2 \eta|<|\xi|+2 \varepsilon_{1}$,

$$
\left|\frac{t}{|t|} \cdot(\xi-2 \eta)\right|>|\xi|-2 \varepsilon_{2}|\xi|-4 \varepsilon_{1}, \quad \text { and } \quad|\xi|>\delta / 4 \text {. }
$$

Now we replace $g_{1}$ in the definition of $T_{1}$ by $\left(1-\rho_{1}\right) g_{1}$ to define $S_{1}$, by 
$\left(1-\rho_{2}\right) \rho_{1} g_{1}$ to define $S_{2}$, by $\rho_{1} \rho_{2}\left(1-\beta_{1}-\beta_{2}\right) g_{1}$ to define $S_{3}$ and by $\rho_{1} \rho_{2} \beta_{i} g_{1}$ to define $S_{3+i}$. Thus $T_{1} f=\sum_{i=1}^{5} S_{i} f$.

Letting $s_{1}(\xi, t)$ denote the kernel of $S_{1}$, we have

$$
s_{1}(\xi, t)=-\frac{1}{4} \int_{\mathbf{R}^{3}} \frac{h_{1}(\xi, \eta, t)}{\eta \cdot t-|t|^{2}+i 0} d \eta
$$

where $h_{1}=\left(1-\rho_{1}\right) g_{1}\left(|\xi-2 \eta|^{2}-|\xi|^{2}\right)^{-1}$. Applying Lemma 4.4, we conclude $|t|\left\|s_{1}(\cdot, t)\right\|_{\alpha^{\prime}}$ is bounded in $t$ for all $\alpha^{\prime}<1$. Since we also have $s_{1}(\xi, t)=0$ for $|t-\xi|>2 M$, we conclude

$$
\left\|\Lambda^{N+1} S_{1} f\right\|_{\alpha^{\prime}} \leqq C_{N}\left\|\Lambda^{N} f\right\|_{0} .
$$

Thus $S_{1}$ is a compact operator on $H_{\alpha, N}$.

Letting $s_{2}(\xi, t)$ denote the kernel of $S_{2}$, we have

$$
s_{2}(\xi, t)=\int_{\mathbf{R}^{3}} \frac{h_{2}(\xi, \eta, t)}{\xi-\left.2 \eta\right|^{2}-(|\xi|+i 0)^{2}} d \eta,
$$

where $h_{2}=\left(1-\rho_{2}\right) \rho_{1} g_{1}\left(-4 \eta \cdot t+4|t|^{2}\right)^{-1}$. Hence, changing variables

$$
s_{2}(\xi, t)=\int_{\mathbf{R}^{3}} \frac{h_{2}\left(\xi, \frac{\eta+\xi}{2}, t\right)}{|\eta|^{2}-(|\xi|+i 0)^{2}} d \eta .
$$

Since $|t| h_{2}(\xi,(\eta+\xi) / 2, t)$ is bounded, vanishes for $|\xi-\eta|>2 M$, and has Lipschitz constant in $(\xi, \eta)$ uniformly bounded in $t$, it follows that $|t|\left\|s_{2}(\cdot, t)\right\|_{\alpha^{\prime}}$ is uniformly bounded in $t$ for some $\alpha^{\prime}>\alpha$. To verify this one can write

$$
s_{2}(\xi, t)=\int_{\mathbf{R}^{3}} \frac{h_{2}\left(\xi, \frac{\eta+\xi}{2}, t\right) \varphi(\eta)}{|\eta|^{2}-(|\xi|+i 0)^{2}} d \eta+\int_{\mathbf{R}^{3}} \frac{h_{2}\left(\xi, \frac{\eta+\xi}{2}, t\right)(1-\varphi(\eta))}{|\eta|^{2}-(|\xi|+i 0)^{2}} d \eta \equiv s_{2,1}+s_{2,2},
$$

where $\varphi \in C_{0}^{\infty}\left(\mathbf{R}^{3}\right)$ satisfying $\varphi(\eta) \equiv 1$ for $|\eta|<1$. Then $\left\|s_{2,1}(\cdot, t)\right\|_{\alpha^{\prime}}$ can be estimated using Theorem 2.2 and the early steps in the proof of Theorem 2.1 and $\left\|s_{2,2}(\cdot, t)\right\|_{\alpha^{\prime}}$ can be estimated directly by Lemma 4.4. Since $s_{2}(\xi, t)=0$ for $|\xi-t|>2 M$, it follows that $S_{2}$ like $S_{1}$ is compact on $H_{\alpha, N}$.

Letting $s_{3}(\xi, t)$ denote the kernel of $S_{3}$, we have

$$
s_{3}(\xi, t)=-\frac{1}{4} \int_{\mathbf{R}^{3}} \frac{h_{3}(\xi, \eta, t)}{\left.|\xi-2 \eta|^{2}-(|\xi|+i 0)^{2}\right)\left(\eta \cdot t-|t|^{2}+i 0\right)} d \eta,
$$

where $h_{3}=\rho_{1} \rho_{2}\left(1-\beta_{1}-\beta_{2}\right) g_{1}$. Since $|\xi|>\delta / 4$ on support $h_{3}$, by taking $\varepsilon_{1}$ sufficiently small we can assume that $|\xi-2 \eta|$ does not vanish on the support of $h_{3}$. Thus all partial derivatives of $h_{3}$ with respect to $\xi$ and $\eta$ are bounded on $\mathbf{R}^{9}$.

We want to use the coordinates $\mu_{1}=|\eta-\xi / 2|$ and $\mu_{2}=(\eta-\xi / 2) \cdot t /|t|$ on $\eta$-space to study $s_{3}$, since the singularities of the integrand are on level surfaces of $\mu_{1}$ and $\mu_{2}$. To see that these coordinates are independent on support $h_{3}$ and estimate derivatives with respect to $\mu_{1}$ and $\mu_{2}$, it is convenient to introduce cylindrical 
coordinates $(\rho, \theta, z)$ with origin $\xi / 2$ and axis in the direction of $t$. Then we have $\rho=\sqrt{\mu_{1}^{2}-\mu_{2}^{2}}$ and $z=\mu_{2}$.

The factor $\rho_{1} \rho_{2}\left(1-\beta_{1}-\beta_{2}\right)$ in $h_{3}$ insures that

$$
\begin{aligned}
\mu_{1}-\left|\mu_{2}\right|= & \mid\left(|\eta-\xi / 2|-\frac{|\xi|}{2}\right)-\left(\left|\frac{\xi \cdot t}{2|t|}-\frac{\eta \cdot t}{|t|}\right|-\left|\frac{\xi \cdot t}{2|t|}-\right| t||\right) \\
& -\left(\left|\frac{\xi \cdot t}{2|t|}-\right| t||-\frac{|\xi|}{2}\right)\left|>\varepsilon_{2}\right| \xi \mid-3 \varepsilon_{1}
\end{aligned}
$$

on support $h_{3}$. Thus, choosing $\varepsilon_{1}$ sufficiently small once $\varepsilon_{2}$ has been fixed, we have

$$
\mu_{1}-\left|\mu_{2}\right| \geqq \varepsilon_{3}|\xi|
$$

on support $h_{3}$. Setting

$$
m_{3}(\rho, z, \xi, t)=\int_{0}^{2 \pi} \frac{h_{3}\left(\xi, \frac{\xi}{2}+z \frac{t}{|t|}+\rho \cos \theta \hat{e}_{1}(t)+\rho \sin \theta \hat{e}_{2}(t), t\right)}{\left(z^{2}+\rho^{2}\right)^{1 / 2}+|\xi|} d \theta
$$

where $\left(t /|t|, \hat{e}_{1}(t), \hat{e}_{2}(t)\right)$ is an orthonormal frame, we see that $|\xi| m_{3}$ is bounded together with its derivatives in $\rho, z$ and $\xi$. We have

$$
s_{3}(\xi, t)=-\frac{1}{4|t|} \int_{\mathbf{R} \times \mathbf{R}_{+}} \frac{m_{3} \rho d \rho d z}{\left(2 \sqrt{\rho^{2}+z^{2}}-(|\xi|+i 0)\right)\left(z+\frac{\xi \cdot t}{2|t|}-|t|+i 0\right)}
$$

and, since $\partial\left(\mu_{1}, \mu_{2}\right) / \partial(\rho, z)=\rho / \mu_{1}$,

$$
s_{3}(\xi, t)=-\frac{1}{4|t|} \int_{\mathbf{R} \times \mathbf{R}_{+}} \frac{\mu_{1} m_{3} d \mu_{1} d \mu_{2}}{\left(2 \mu_{1}-(|\xi|+i 0)\right)\left(\mu_{2}+\frac{\xi \cdot t}{2|t|}-|t|+i 0\right)} .
$$

Since $m_{3}$ is supported in

$$
\left\{\left|2 \mu_{1}-\right| \xi||<2 \varepsilon_{1}\right\} \cap\left\{\left|\mu_{2}+\frac{\xi \cdot t}{2|t|}-\right| t||<2 \varepsilon_{1}\right\},
$$

we have $\mu_{1}^{2}-\mu_{2}^{2}>\varepsilon_{3}|\xi| \mu_{1}>\varepsilon_{3}\left(2 \mu_{1}-2 \varepsilon_{1}\right) \mu_{1}$ on support $m_{3}$. Thus all partial derivatives of $\rho$ with respect to $\mu_{1}$ and $\mu_{2}$ are bounded on support $m_{3}$, and $\mu_{1} m_{3}$ and its derivatives in $\mu$ and $\xi$ are bounded. Thus, applying Lemma 4.4 twice, one sees that $|t|\left\|s_{3}(\cdot, t)\right\|_{\alpha^{\prime}}$ is bounded on $\mathbf{R}^{3}$ for $\alpha^{\prime}<1$, and, since $s_{3}(\xi, t)=0$ for $|\xi-t|>2 M$, it follows that $S_{3}$ is compact on $H_{\alpha, N}$.

The kernels $s_{4}$ and $s_{5}$ of $S_{4}$ and $S_{5}$ require a more detailed analysis. We have for $i=4,5$,

$$
s_{i}(\xi, t)=\int_{\mathbf{R}^{3}} \frac{h_{i}(\xi, \eta, t)}{\left(|\xi-2 \eta|^{2}-(|\xi|+i 0)^{2}\right)\left(|\eta-2 t|^{2}-(|\eta|+i 0)^{2}\right)} d \eta,
$$

where $h_{i}=\rho_{1} \rho_{2} \beta_{i} g_{1}$. Thus, as for $h_{3}, h_{i}$ has bounded derivatives with respect to $(\xi, \eta)$ of all orders. Moreover, writing $t$ in spherical coordinates, one sees that $h_{i}, i=4,5$, as bounded derivatives of all orders as a function on $\mathbf{R}^{3} \times \mathbf{R}^{3} \times \overline{\mathbf{R}}_{+} \times S^{2}$. 
We let $\mu_{1}$ be the $t$-component of $2 \eta-\xi$, i.e. $\mu_{1}=((2 \eta-\xi) t)|t|^{-1}$, and $\mu_{2}$ be the projection of $2 \eta-\xi$ on the plane orthogonal to $t$, i.e.

$$
\mu_{2}=2 \eta-\xi-\frac{(2 \eta-\xi) \cdot t}{|t|^{2}} t
$$

By our choices of $\varepsilon_{1}$ and $\varepsilon_{2},\left|\mu_{2}\right|<1 / 2|\xi|$ on support $h_{i}$, and, since $h_{i}=0$ if $|\xi-\eta|>M$ or $|t-\eta|>M$, we also have $\left|\mu_{2}\right|<2 M$ on support $h_{i}$.

We set $\mu_{1}(\xi, t)=2|t|-(\xi \cdot t)|t|^{-1}$, expand $s_{i}(\xi, t)$ as

$$
\begin{aligned}
s_{i}(\xi, t)= & \frac{1}{2} \int_{\mathbf{R}^{3}} \frac{h_{i}-h_{i} \uparrow_{\mu_{1}=\mu_{1}(\xi, t)}}{\left(\mu_{1}^{2}+\left|\mu_{2}\right|^{2}-(|\xi|+i 0)^{2}\right)(2|t|)\left(\mu_{1}(\xi, t)-\mu_{1}\right)} d \mu_{1} d m \\
& +\frac{1}{2} \int_{\mathbf{R}^{3}} \frac{h_{i} \uparrow_{\mu_{1}=\mu_{1}(\xi, t)}}{\left(\mu_{1}^{2}+\left|\mu_{2}\right|^{2}-(|\xi|+i 0)^{2}\right)(2|t|)\left(\mu_{1}(\xi, t)-\mu_{1}-i 0\right)} d \mu_{1} d m \\
\equiv & s_{i, 1}+s_{i, 2},
\end{aligned}
$$

where $d m$ is Lebesgue measure on $t \cdot \eta=0$. We consider $s_{i, 1}$ as a function of the form (4.10) with

$$
g=\frac{1}{4} \frac{h_{i}(\xi, \eta, t)-h_{i} \uparrow_{\mu_{1}=\mu_{1}(\xi, t)}}{(|\eta|+|\xi|)\left(\mu_{1}(\xi, t)-\mu_{1}\right)}
$$

Although $g$ is not supported in a bounded set, it has bounded support in $\mu_{2}$ and the expansion used in Lemma 4.4 shows that (4.11) holds for $s=|t| s_{i, 1}$. Thus, since $s_{i, 1}(\xi, t)=0$ for $|\xi-t|>2 M, s_{i, 1}$ is the kernel of a compact integral operator on $H_{\alpha, N}$.

We evaluate $s_{i, 2}$ by computing the integral in $\mu_{1}$ by residues (there is a simple pole in $\operatorname{Im} \mu_{1}>0$ at $\mu_{1}=\sqrt{(|\xi|+i 0)^{2}-\left|\mu_{2}\right|^{2}}$ ). This gives

$$
|t| s_{i, 2}(\xi, t)=\int_{\mathbf{R}^{2}} \frac{k_{i}\left(\mu_{2}, \xi, t\right)}{\sqrt{|\xi|^{2}-\left|\mu_{2}\right|^{2}}\left(2|t|-\frac{\xi \cdot t}{|t|}-\sqrt{|\xi|^{2}-\left|\mu_{2}\right|^{2}}-i 0\right)} d \mu_{2},
$$

where

$$
k_{i}=\frac{\pi i}{2} h_{i} \uparrow_{\mu_{1}=2|t|-\xi \cdot t|| t \mid}
$$

On the support of $h_{5}, 2|t|-(\xi \cdot t)|t|^{-1}<-|\xi|+2 \varepsilon_{2}|\xi|,|\xi|>\delta / 4$ and $\left|\mu_{2}\right|<\min \{|\xi| / 2$, $2 M\}$. Thus the integrand defining $s_{5,2}$ is smooth in $\xi$ with bounded support in $\mu_{2}$. Since one has $|t|\left|\partial_{\xi}^{\beta} s_{5,2}(\xi, t)\right|$ bounded for all $\beta$ and $s_{5,2}(\xi, t)=0$ for $|\xi-t|>2 M$, the integral operator corresponding to $s_{5,2}$ is compact.

To simplify the study of $s_{4,2}$ we use polar coordinates in the plane $\eta \cdot t=0$ and set $m_{4}\left(\left|\mu_{2}\right|^{2}, \xi, t\right)=\int_{0}^{2 \pi} k_{4}\left(\left|\mu_{2}\right| \cos \theta,\left|\mu_{2}\right| \sin \theta, \xi, t\right) d \theta$. It is important that $m_{4}$ is a smooth function of $\left|\mu_{2}\right|^{2}$ on $\overline{\mathbf{R}}_{+}$-note that only homogeneous functions of $(\cos \theta, \sin \theta)$ of even degree survive the integration. Thus $m_{4}(s, \xi, t)$ is smooth in $(s, \xi)$ on $\overline{\mathbf{R}}_{+} \times \mathbf{R}^{3}$ and its partial derivatives with respect to $s$ and $\xi$ are bounded on $\overline{\mathbf{R}}_{+} \times \mathbf{R}^{3} \times \mathbf{R}^{3}$. It also remains true that, if we write $t$ in spherical coordinates, $m_{4}$ is smooth on $\overline{\mathbf{R}}_{+} \times \mathbf{R}^{3} \times \overline{\mathbf{R}}_{+} \times S^{2}$. 
We now have

$$
s_{4,2}(\xi, t)=\lim _{\varepsilon \downarrow 0} \frac{1}{2|t|} \int_{0}^{\infty} \frac{m_{4}(s, \xi, t)}{\sqrt{|\xi|^{2}-s\left(2|t|-\frac{\xi \cdot t}{|t|}-\sqrt{|\xi|^{2}-s}-i \varepsilon\right)}} d s
$$

and integrating by parts gives

$$
\begin{aligned}
s_{4,2}(\xi, t)= & -\frac{m_{4}(0, \xi, t) \ln \left(2|t|-\frac{\xi \cdot t}{|t|}-|\xi|-i 0\right)}{|t|} \\
& -\frac{1}{|t|} \int_{0}^{\infty} \frac{\partial m_{4}}{\partial s} \ln \left(2|t|-\frac{\xi \cdot t}{|t|}-\sqrt{|\xi|^{2}-s}-i 0\right) d s \equiv v_{4,1}+v_{4,2} .
\end{aligned}
$$

Note that the integration in $v_{4,2}$ is over $0<s<(2 M)^{2}$ and $|2| t|-(\xi \cdot t) /| t|-| \xi||<$ $2 \varepsilon_{2}|\xi|$ on support $m_{4}$. The kernel $v_{4,2}$ is superposition of the kernels

$$
w_{a}(\xi, t)=\frac{m(a, \xi, t) \ln \left(2|t|-(\xi \cdot t)|t|^{-1}-\sqrt{|\xi|^{2}-a}-i 0\right)}{|t|}
$$

for $0 \leqq a \leqq a_{0}$, where $m(a, \xi, r \omega)$ is smooth on $\overline{\mathbf{R}}_{+} \times \mathbf{R}^{3} \times \overline{\mathbf{R}}_{+} \times S^{2}$ with bounded derivatives in $\xi$ and $r$ and $m=0$ for $|\xi-t|>2 M$ and for $|\xi|^{2}<\max \left\{4 a, \delta^{2} / 16\right\}$. The kernel $v_{4,1}$ is $w_{0}(\xi, t)$ with $m(0, \xi, t)=-m_{4}(0, \xi, t)$. Thus to complete the proof that $T_{1}$ is compact it will suffice to show for $W_{a}$ with kernel $w_{a}$,

$$
\left\|\Lambda^{N+1} W_{a} f\right\|_{1} \leqq C_{\alpha}\left\|\Lambda^{N} f\right\|_{\alpha}
$$

for all $\alpha>0$ with $C^{\alpha}$ uniform on $0 \leqq a \leqq a_{0}$.

We have

$$
\left|\Lambda^{N+1}(\xi) W_{a} f(\xi)\right| \leqq C \int_{|\xi-t|<2 M}\left|\ln \left(2|t|-(\xi \cdot t)|t|^{-1}-\sqrt{|\xi|^{2}-a}-i 0\right)\right| d t\left\|\Lambda^{N} f\right\|_{0}
$$

and, since the integral is bounded uniformly for $(a, \xi) \in\left[0, a_{0}\right] \times \mathbf{R}^{\mathbf{3}}$, this gives

$$
\sup \left|\Lambda^{N+1}(\xi) W_{a} f(\xi)\right| \leqq C\left\|\Lambda^{N} f\right\|_{0} .
$$

To estimate the Lipschitz norm of $\Lambda^{N+1}(\xi) W_{a} f(\xi)$, we use $\varphi(s) \in C^{\infty}(\mathbf{R})$, satisfying $\varphi(s)=1$ for $|s|<1$ and $\varphi(s)=0$ for $|s|>2$, to write

$$
W_{a} f(\xi)=\int_{\mathbf{R}^{3}} \varphi(|t|) w_{a}(\xi, t) f(t) d t+\int_{\mathbf{R}^{3}}(1-\varphi(|t|)) w_{a}(\xi, t) f(t) d t \equiv I_{1}+I_{2} .
$$

In $I_{1}$ we will use spherical coordinates, $t=r \omega$. Since $m(a, \xi, r \omega)$ is smooth in $r$ uniformly in $(a, \xi, \omega)$, extending $r m(a, \xi, r \omega)$ to be zero for $r<0$ gives a Lipschitz function of $r$ uniformly in $\omega$, which we denote by $\tilde{m}(a, \xi, r, \omega)$. Thus

$$
I_{1}=\int_{S^{2}} d \omega \int_{\mathbf{R}} \varphi(r) \tilde{m} \ln \left(2 r-(\xi \cdot \omega)-\sqrt{|\xi|^{2}-a}-i 0\right) f(r, \omega) d r
$$

where for $r \geqq 0 f( \pm r, \omega)=f(r \omega)$. Expanding $I_{1}$ as in the proof of Lemma 4.4, one sees that for $f \in C^{\alpha}, \alpha>0, I_{1}$ is differentiable in $\xi$ with 


$$
\begin{aligned}
\frac{\partial I_{1}}{\partial \xi_{i}}= & \int_{S^{2}} d \omega \int_{\mathbf{R}} \varphi(r) \frac{\partial \tilde{m}}{\partial \xi_{i}} \ln \left(2 r-(\xi \cdot \omega)-\sqrt{|\xi|^{2}-a}-i 0\right) f(r, \omega) d r \\
& +\int_{S^{2}} d \omega \int_{\mathbf{R}}-\frac{\left(\omega_{i}+\xi_{i}\left(|\xi|^{2}-a\right)^{-1 / 2}\right) \varphi(r) \tilde{m} f(r, \omega)}{2 r-(\xi \cdot \omega)-\sqrt{|\xi|^{2}-a}-i 0} d r \equiv J_{1}+J_{2}
\end{aligned}
$$

One has

$$
\left|J_{1}(\xi)\right| \leqq C\|f\|_{0}
$$

by the reasoning that gave (4.14) and

$$
\left|J_{2}(\xi)\right| \leqq C\|f\|_{\alpha^{\prime}}
$$

for any $\alpha^{\prime}>0$ by Lemma 4.4 applied to the integral over R. Since $|\xi|$ is bounded on support $\varphi(|t|) m$, we have

$$
\left|\Lambda^{N+1}(\xi) I_{1}(\xi, a)\right| \leqq C\|f\|_{\alpha^{\prime}}
$$

for any $\alpha^{\prime}>0$.

Since all functions are smooth in $t$ for $|t| \geqq 1$, the expansion used in the proof of Lemma 4.4 can be used to show that $I_{2}$ is differentiable in $\xi$ with

$$
\frac{\partial I_{2}}{\partial \xi_{i}}=\int_{\mathbf{R}^{3}}-\frac{\left(t_{i}|t|^{-1}+\xi_{i}\left(|\xi|^{2}-a\right)^{-1 / 2}\right)(1-\varphi(|t|)) m f(t)}{\left(2|t|-(\xi \cdot t)|t|^{-1}-\sqrt{|\xi|^{2}-a}-i 0\right)|t|} d t
$$

and, since $\xi$ and $t$ have comparable magnitudes on support $(1-\varphi(|t|) m)$, Lemma 4.4 shows

$$
\sup \left|\Lambda^{N+1}(\xi) \frac{\partial I_{2}}{\partial \xi_{i}}\right| \leqq C\left\|\Lambda^{N} f\right\|_{\alpha^{\prime}}
$$

for any $\alpha^{\prime}>0$. Combining (4.14)-(4.16) gives (4.13), and completes the proof that $T_{1}$ is compact on $H_{\alpha, N}$.

In terms of $\eta$ the integrand defining $T_{2}$ is singular on the spheres (for $\xi \neq 0$ )

$$
\Pi_{1}=\{\eta:|\eta|=|\xi|\}
$$

and

$$
\Pi_{2}=\{\eta:|\eta-2 t|=|\xi|\} .
$$

At $t=0$ these spheres coincide but the most singular part of the kernel $t_{2}(\xi, t)$ of $T_{2}$ is the set corresponding to tangency of $\Pi_{1}$ and $\Pi_{2}$, i.e.

$$
|t|=|\xi| \text {. }
$$

As in the proof of the compactness of $T_{1}$, we introduce a partition of unity adapted to these sets generated by

$$
\rho_{1}=\rho(|\eta|-|\xi|) \text { and } \rho_{2}=\rho(|\eta-2 t|-|\xi|),
$$

where $\rho(s)=1$ for $|s|<\varepsilon_{1}$ and $\rho \in C_{0}^{\infty}\left(|s|<2 \varepsilon_{1}\right)$. Again for $\varepsilon_{1}$ sufficiently small one has $|\xi|>\delta / 4$ on the support of $\rho_{1} \rho_{2} g_{2}$, and $\rho_{1} \rho_{2} g_{2}$ is smooth. The cutoffs corresponding to the more singular parts are 


$$
\beta_{1}=\beta\left(\frac{|t|}{|\xi|}\right) \text { and } \beta_{2}=\beta\left(\frac{|t|-|\xi|}{|\xi|}\right),
$$

where $\beta(s)=1$ for $|s|<\varepsilon_{2}$ and $\beta \in C_{0}^{\infty}\left(|s|<2 \varepsilon_{2}\right)$. Note that for $\varepsilon_{2}$ sufficiently small $\beta_{1}$ and $\beta_{2}$ are smooth on support $\rho_{1} \rho_{2} g_{2}$.

Next we define $S_{1}, \ldots, S_{5}$ precisely as in the proof for $T_{1}$. Thus the integrand of $S_{1}$ vanishes on a neighborhood of $\Pi_{1}$, the integrand of $S_{2}$ vanishes on a neighborhood of $\Pi_{2}$, and on the support of the integrand of $S_{3}$ we can introduce coordinates for which $\Pi_{1}$ and $\Pi_{2}$ are level sets. These three terms are treated exactly as before: in place of $|t|$ the weight factor in the denominator is $|\eta-2 t|+|\xi|$.

For $S_{4}$ we introduce spherical coordinates in $\eta, \eta=r \omega,|\omega|=1, r>0$. Then

$$
\begin{aligned}
|\eta-2 t|^{2}-|\xi|^{2}= & r^{2}-4 r \omega \cdot t+4|t|^{2}-|\xi|^{2} \\
= & \left(r-2 t \cdot \omega-\sqrt{|\xi|^{2}+4(t \cdot \omega)^{2}-4|t|^{2}}\right) \\
& \left(r-2 t \cdot \omega+\sqrt{|\xi|^{2}+4(t \cdot \omega)^{2}-4|t|^{2}}\right),
\end{aligned}
$$

and for $\varepsilon_{2}$ sufficiently small $|\xi|^{2}-8|t|^{2}>1 / 2|\xi|^{2}$ on support $h_{4}$. Thus, the kernel $s_{4}(\xi, t)$ of $S_{4}$ given by

$$
s_{4}(\xi, t)=\int_{S^{2}} d \omega \int_{0}^{\infty} \frac{k_{4}(\xi, r, \omega, t)}{(r-|\xi|-i 0)\left(r-2 t \cdot \omega-\sqrt{|\xi|^{2}+4(t \cdot \omega)^{2}-4|t|^{2}}-i 0\right)} d r
$$

where

$$
k_{4}=\frac{r^{2} h_{4}}{r+|\xi|}\left(r-2 t \cdot \omega+\sqrt{|\xi|^{2}+4(t \cdot \omega)^{2}-4|t|^{2}}\right)^{-1}
$$

is a smooth function on $\mathbf{R}^{3} \times \overline{\mathbf{R}}_{+} \times S^{2} \times \mathbf{R}^{3}$.

Expanding in the usual manner, we have (with $A=|\xi|^{2}+4(t \cdot \omega)^{2}-4|t|^{2}$ )

$$
\begin{aligned}
s_{4}(\xi, t)= & \int_{S^{2}} d \omega \int_{0}^{\infty} \frac{k_{4}(\xi, r, \omega, t)-k_{4}(\xi,|\xi|, \omega, t)}{(r-|\xi|)(r-2 t \cdot \omega-\sqrt{A}-i 0)} d r \\
& +\int_{S^{2}} d \omega \int_{0}^{\infty} \frac{k_{4}(\xi,|\xi|, \omega, t)}{(r-|\xi|-i 0)(r-2 t \cdot \omega-\sqrt{A}-i 0)} d r \\
\equiv & s_{4,1}(\xi, t)+s_{4,2}(\xi, t) .
\end{aligned}
$$

The main point here is that, since the integral in $s_{4,2}$ is the limit as $\varepsilon \downarrow 0$ of the same integral with $i 0$ replaced by $i \varepsilon$, we can deform the integration on $[0, \infty)$ to a contour in the upper half plane-for instance $z=r(1+i)$. Since $|\xi|>\delta / 4$ and $|\xi-t|<2 M$ on support $k_{4}$, this shows $s_{4,2}(\xi, t) \in C_{0}^{\infty}\left(\mathbf{R}^{6}\right)$. Since

$$
\frac{k_{4}(\xi, r, \omega, t)-k_{4}(\xi,|\xi|, \omega, t)}{r-|\xi|}
$$

is a smooth function supported on

$$
\left\{|\xi|>\delta_{0} / 4\right\} \cap\{|\xi-t|<2 M\} \cap\left\{|t|<2 \varepsilon_{2}|\xi|\right\} \cap\left\{|r-| \xi||<2 \varepsilon_{1}\right\},
$$

it follows that $s_{4,1}(\xi, t)$ also has compact support, and it has enough regularity in 
$\xi$ that the corresponding integral operator is compact on $H_{\alpha, N}$. Thus $S_{4}$ is compact.

For $S_{5}$ we introduce the $t$-component of $\eta \mu_{1}=\eta \cdot t /|t|$, and set $\mu_{2}=\eta-\left(\eta \cdot t /|t|^{2}\right) t$. Since $|t-\eta|<M$ on support $h_{5}$, we have $\left|\mu_{2}\right|<M$ on support $h_{5}$. Moreover, since we also have

$$
|| \xi|-| t||<2 \varepsilon_{2}|\xi|, \quad|| \eta|-| \xi||<2 \varepsilon_{1}, \quad|| \eta-2 t|-| \xi||<2 \varepsilon_{1}
$$

and $|\xi|>\delta_{0} / 4$ on support $h_{5}$, it also follows that, choosing $\varepsilon_{1}$ and $\varepsilon_{2}$ sufficiently small we can make $\left|\mu_{2}\right|<1 / 2|\xi|$ on support $h_{5}$. The kernel of $S_{5}$ is given by

$$
\begin{aligned}
s_{5}(\xi, t) & =\int_{\mathbf{R}^{3}} \frac{h_{5}(\xi, \eta, t) d \eta}{\left(\mu_{1}^{2}+\left|\mu_{2}\right|^{2}-|\xi|^{2}-i 0\right)\left(\left(\mu_{1}-2|t|\right)^{2}+\left|\mu_{2}\right|^{2}-|\xi|^{2}-i 0\right)} \\
& =\int_{\mathbf{R}^{3}} \frac{k_{5}(\xi, \eta, t) d \eta}{\left(\mu_{1}-\sqrt{|\xi|^{2}-\left|\mu_{2}\right|^{2}}-i 0\right)\left(\mu_{1}-2|t|+\sqrt{|\xi|^{2}-\left|\mu_{2}\right|^{2}}+i 0\right)}
\end{aligned}
$$

where

$$
k_{5}=\frac{h_{5}}{\left(\mu_{1}+\sqrt{|\xi|^{2}-\left|\mu_{2}\right|^{2}}\right)\left(\mu_{1}-2|t|-\sqrt{|\xi|^{2}-\left|\mu_{2}\right|^{2}}\right)} .
$$

Note that, since for $\varepsilon_{1}$ and $\varepsilon_{2}$ sufficiently small one has $\left|\mu_{1}-\right| t||<1 / 4|\xi|$ and $|t|>3 / 4|\xi|$ on support $h_{5}, k_{5}$ is a smooth function satisfying $(1+|\xi|)^{2}\left|\partial_{\xi, \eta, t}^{\beta} k_{5}\right| \leqq C$ for all $\beta$.

Expanding $s_{5}(\xi, t)$, we have

$$
\begin{aligned}
s_{5}(\xi, t)= & \int_{\mathbf{R}^{3}} \frac{\left(k_{5}(\xi, \eta, t)-k_{5}\right) \uparrow_{\mu_{1}=\sqrt{ }|\xi|^{2}-\left|\mu_{2}\right|^{2}}}{\left(\mu_{1}-\sqrt{|\xi|^{2}-\left|\mu_{2}\right|^{2}}\right)\left(\mu_{1}-2|t|+\sqrt{|\xi|^{2}-\left|\mu_{2}\right|^{2}}+i 0\right)} d \eta \\
& +\int_{\mathbf{R}^{2}} d \mu_{2} \int_{\mathbf{R}} \frac{\left.k_{5}\right|_{\mu_{1}=\sqrt{ }|\xi|^{2}-\left|\mu_{2}\right|^{2}}}{\left(\mu_{1}-\sqrt{|\xi|^{2}-\left|\mu_{2}\right|^{2}}-i 0\right)\left(\mu_{1}-2|t|+\sqrt{|\xi|^{2}-\left|\mu_{2}\right|^{2}}+i 0\right)} d \mu_{1} \\
\equiv & s_{5,1}(\xi, t)+s_{5,2}(\xi, t) .
\end{aligned}
$$

From the restrictions on the support of $h_{5}$ one sees that $s_{5,1}$ is a smooth function supported in $|\xi-t|<2 M$, satisfying $\sup (1+|\xi|)\left|\partial_{\xi}^{\beta} s_{5,1}(\xi, t)\right|<\infty$, for all $\beta$. Thus the integral operator corresponding to $s_{5,1}$ is compact on $H_{\alpha, N}$.

Calculating the integral in $\mu_{1}$ in $s_{5,2}$ by residues, we have

$$
s_{5,2}(\xi, t)=\pi i \int_{\mathbf{R}^{2}} \frac{k_{5} \uparrow_{\mu_{1}}=\sqrt{|\xi|^{2}}-\left|\mu_{2}\right|^{2}}{\sqrt{|\xi|^{2}-\left|\mu_{2}\right|^{2}}-|t|+i 0} d \mu_{2} .
$$

Multiplying numerator and denominator by $\sqrt{|\xi|^{2}-\left|\mu_{2}\right|^{2}}+|t|$, which is smooth on support $h_{5}$, we have

$$
s_{5,2}=\pi i \int_{\mathbf{R}^{2}} \frac{l_{5}\left(\xi, t, \mu_{2}\right)}{|\xi|^{2}-\left|\mu_{2}\right|^{2}-|t|^{2}+i 0} d \mu_{2}
$$

where $l_{5}=\left(\sqrt{|\xi|^{2}-\left|\mu_{2}\right|^{2}}+|t|\right) k_{5} \mid \mu_{1}=\sqrt{|\xi|^{2}-\left|\mu_{2}\right|^{2}}$. Note that $m_{5}=\pi i / 2 \int_{0}^{2 \pi} l_{5} d \theta$ is a smooth function of $\left(\xi, r^{2}, t\right)$ supported in $\{|t-\xi|<2 M\} \cap\{r<M\}$, satisfying 


$$
\begin{aligned}
& \sup (1+|\xi|)\left|\partial_{\xi, r, t}^{\beta} m_{5}\right|<\infty \text {. Thus } \\
& s_{5,2}(\xi, t)=2 \int_{0}^{\infty} \frac{m_{5}\left(\xi, r^{2}, t\right)}{|\xi|^{2}-r^{2}-|t|^{2}+i 0} r d r=-\int_{0}^{\infty} \frac{m_{5}(\xi, s, t)}{s-|\xi|^{2}+|t|^{2}-i 0} d s \\
& =m_{5}(\xi, 0, t) \ln \left(|t|^{2}-|\xi|^{2}-i 0\right)+\int_{0}^{\infty} \frac{\partial m_{5}}{\partial s}(\xi, s, t) \ln \left(s+|t|^{2}-|\xi|^{2}-i 0\right) d s \\
& =m_{5}(\xi, 0, t) \ln (|t|-|\xi|-i 0)+\int_{0}^{\infty} \frac{\partial m_{5}}{\partial s}(\xi, s, t) \ln \left(\left(s+|t|^{2}\right)^{1 / 2}-|\xi|-i 0\right) d s \\
& +m_{5}(\xi, 0, t) \ln (|t|+|\xi|)+\int_{0}^{\infty} \frac{\partial m_{5}}{\partial s}(\xi, s, t) \ln \left(\left(s+|t|^{2}\right)^{1 / 2}+|\xi|\right) d s \\
& \equiv v_{1}+v_{2}+v_{3}+v_{4},
\end{aligned}
$$

The kernels $v_{3}$ and $v_{4}$ are supported in $|\xi-t|<2 M$ and they satisfy $\left\|\Lambda^{s}(\cdot) v_{i}(\cdot, t)\right\|_{1}<$ $C, t \in \mathbf{R}^{3}, i=3,4$, for $s+1$. Thus the corresponding integral operators, $V_{3}$ and $V_{4}$, are compact on $H_{\alpha, N}$.

The remaining terms in $T_{2} f, V_{1} f$ and $V_{2} f$, are super positions of the operators

$$
\left[V_{a} f\right](\xi)=\int_{\mathbf{R}^{3}} m_{5}(\xi, a, t) \ln \left(\left(a+|t|^{2}\right)^{1 / 2}-|\xi|-i 0\right) f(t) d t
$$

for $0 \leqq a \leqq M$.

Since $t$ and $\xi$ are bounded away from zero on the support of $m_{5}$, the expansion used in the proof of Lemma 4.4 again shows that $V_{1} f$ is differentiable and

$$
\frac{\partial V_{a} f}{\partial \xi_{i}}=\int_{\mathbf{R}^{3}} \frac{\partial m_{5}}{\partial \xi_{i}} \ln \left(\left(a+|t|^{2}\right)^{1 / 2}-|\xi|-i 0\right) f(t) d t+\int_{\mathbf{R}^{3}} m_{5}\left(-\frac{\xi_{i}|\xi|^{-1} f(t)}{\left(a+|t|^{2}\right)^{1 / 2}-|\xi|-i 0}\right) d t .
$$

Thus, since $\xi$ and $t$ have comparable magnitude on the support of $m_{5}, V_{a}$ satisfies the estimate (4.13), i.e.

$$
\left\|\Lambda^{N+1}(\xi) V_{a}\right\|_{1} \leqq C_{\alpha^{\prime}}\left\|\Lambda^{N} f\right\|_{\alpha^{\prime}}
$$

for any $\alpha^{\prime}>0$. Thus $V_{a}$ is compact on $H_{\alpha, N}$.

As we showed earlier, Theorem 4.3 has the following corollary.

Corollary 4.5. The Frechet derivative of the backscattering map, dS( $(\hat{q})$, is a Fredholm operator on $H_{\alpha, N}$ of index zero for $\hat{q} \in \mathcal{O}$.

\section{Section 5. Local Invertibility of the Backscattering Map}

In this section we present the consequences of the results of Sects. 3 and 4 for the inverse backscattering problem. The extent of the connected component of 0 containing the zero potential is of interest here. We can show that the intersection of $\mathcal{O}$ with $H_{\alpha, N}^{r}$ is contained in a connected component of $\mathcal{O}$. The proof of that fact requires the following pair of lemmas.

Lemma 5.1. For some $\alpha^{\prime}, \alpha<\alpha^{\prime}<1$, let $\hat{q}(t)$ be a curve in $H_{\alpha^{\prime}, N}$ continuous in the topology of $H_{\alpha^{\prime}, N}$, such that $q(t)$ is a real-valued function in $C_{0}^{\infty}\left(\mathbf{R}^{3}\right)$ for all $t$. Assume 
that $I+A(\hat{q}(0), 0)$ has a one-dimensional nullspace, and that $I+A(\hat{q}(t), 0)$ is invertible for $t \neq 0$. Then, given $\delta>0$, there is a curve $q_{1}(t)$ in $C_{0}^{\infty}\left(\mathbf{R}^{3}\right)$ continuous in the topology of $H_{\alpha^{\prime}, N}$, such that

(i) $q_{1}(t)=q(t)$ for $|t|>\delta$, and

(ii) $I+A\left(\hat{q}_{1}(t), k\right)$ is invertible for all $t$ and $k \geqq 0$, i.e. $\hat{q}_{1}(t) \in \mathcal{O}$ for all $t$.

Proof. Let $\hat{f}(\hat{q}, k) \neq 0$ be an element of the range of the projection:

$$
P(\hat{q}, k)=\frac{1}{2 \pi i} \int_{|\omega+1|=c}(A(\hat{q}, k)-\omega I)^{-1} d \omega .
$$

Since $\alpha^{\prime}>\alpha$, for $\hat{q}$ in $H_{\alpha^{\prime}, N}, A(\hat{q}, k)$ is continuous in $(\hat{q}, k)$ in operator norm on $H_{\alpha, N}$ and compact on $H_{\alpha, N}$ by Theorems 2.1 and 2.2 (see 3.4). Thus it follows that for $c$ sufficiently small $P$ has 1 -dimensional range and is continuous in $(\hat{q}, k)$ on $\left\{\|\hat{q}-\hat{q}(0)\|_{\alpha^{\prime}, N}<c_{1}, 0 \leqq k \leqq c_{1}\right\}$ for $c_{1}$ sufficiently small. Moreover, $P(\hat{q}, k)$ is differentiable in $\hat{q}$ and $\partial P / \partial \hat{q}$ is also continuous in $(\hat{q}, k)$.

We have

$$
(I+A(\hat{q}, k)) \hat{f}(\hat{q}, k)=\lambda(\hat{q}, k) \hat{f}(\hat{q}, k),
$$

where $\lambda(\hat{q}, k) \in \mathbf{C}$ and $\lambda$ has the regularity of $P$. Evaluating $(5.2)$ at $(\hat{q}, k)=(z \hat{q}(0), 0)$, differentiating with respect to $z$, evaluating at $z=1$, and taking the inner product with $[\hat{f}(\hat{q}(0), 0)](\xi)|\xi|^{-2}$, we have (see Remark after Theorem 3.1)

$$
\left.\frac{\partial \lambda(z \hat{q}(0), 0)}{\partial z}\right|_{z=1}=-1 \text {. }
$$

We split $H_{\alpha^{\prime}, N}$ into the direct sum of span $\hat{q}(0)$ and

$$
H^{\prime}=\left\{\hat{q} \in H_{\alpha^{\prime}, N}: \int_{\mathbf{R}^{3} \mathbf{R}^{3}} \hat{q}(\xi-\eta) \frac{\hat{f}_{0}(\xi) \hat{f}_{0}(\eta)}{|\eta|^{2}|\xi|^{2}} d \xi d \eta=0\right\},
$$

where $\hat{f}_{0}=\hat{f}(\hat{q}(0), 0)$.

Let $\lambda\left(\hat{q}^{\prime}, z, k\right)=\lambda\left(\hat{q}^{\prime}+z \hat{q}(0), k\right)$. We consider $\lambda$ as a function on $H^{\prime} \times\{|z-1|<\delta\} \times$ $\{0 \leqq k \leqq \delta\}$. By the implicit function theorem there is an $\varepsilon>0$ such that for $\left\|\hat{q}^{\prime}\right\|_{\alpha^{\prime}, N}<\varepsilon, 0 \leqq k \leqq \varepsilon$ the unique solution to $\lambda\left(\hat{q}^{\prime}, z, k\right)=0$ in $|1-z|<\varepsilon$ is given by $z=z\left(\hat{q}^{\prime}, k\right)$ and $z\left(\hat{q}^{\prime}, k\right)$ is continuous in $\left(\hat{q}^{\prime}, k\right)$. Note that $\lambda\left(\hat{q}^{\prime}, z, k\right)=0$ means $\operatorname{Null}\left(I+A\left(z \hat{q}(0)+\hat{q}^{\prime}, k\right)\right) \neq\{0\}$.

Now suppose $\hat{q}\left(t_{i}\right)=\hat{q}_{i}^{\prime}+z_{i} \hat{q}(0), i=1,2$, with $t_{1}<0, t_{2}>0,\left\|\hat{q}_{i}^{\prime}\right\|_{\alpha^{\prime}, N}<\varepsilon^{\prime}<\varepsilon$ and $\left|z_{i}-1\right|<\varepsilon^{\prime}$. By hypothesis this will hold for $\left|t_{i}\right|<\delta, \delta$ sufficiently small. Also by hypothesis $z_{i} \in \mathbf{R}$ and $z\left(\hat{q}_{i}^{\prime}, 0\right) \neq z_{i}$. Since $q(t)$ is real valued, $q_{1}^{\prime}$ and $q_{2}^{\prime}$ are real, and hence $z\left((1-s) \hat{q}_{1}^{\prime}+s \hat{q}_{2}^{\prime}, k\right)$ does not intersect the real-axis for $(s, k) \in[0,1] \times(0, \varepsilon]$. Thus we may choose $z(t)$ with $z\left(t_{1}\right)=z_{1}$ and $z\left(t_{2}\right)=z_{2}$ with $z(t)$ for $t \in\left(t_{1}, t_{2}\right)$ lying in the half-plane, $\{\operatorname{Im} z>0\}$ or $\{\operatorname{Im} z<0\}$, which does not intersect $\left\{z\left((1-s) \hat{q}_{1}^{\prime}+s \hat{q}_{2}^{\prime}, k\right):(s, k) \in[0,1] \times[0, \varepsilon]\right\}$. Then we define

$$
\hat{q}_{1}(t)=\left(1-\frac{t-t_{1}}{t_{2}-t_{1}}\right) \hat{q}_{1}^{\prime}+\frac{t-t_{1}}{t_{2}-t_{1}} \hat{q}_{2}^{\prime}+z(t) \hat{q}(0)
$$

for $t \in\left[t_{1}, t_{2}\right]$. Finally, we note that we may construct $\hat{q}_{1}(t)$ so that

$$
\left\|\hat{q}_{1}(t)-\hat{q}(0)\right\|_{\alpha^{\prime}, N} \leqq\left(2+\|\hat{q}(0)\|_{\alpha^{\prime}, N}\right) \varepsilon^{\prime}
$$


for $\left[t_{1}, t_{2}\right]$. Thus, taking $\varepsilon^{\prime}$ sufficiently small, we will have $I+A\left(\hat{q}_{1}(t), k\right)$ invertible for $k \geqq \varepsilon, t \in\left[t_{1}, t_{2}\right]$. For $t \notin\left[t_{1}, t_{2}\right]$ we set $q_{1}(t)=q(t)$.

Lemma 5.2. Suppose that $I+A\left(\hat{q}_{0}, 0\right)$ has a kernel of dimension $m>0$, for some $\hat{q}_{0} \in H_{\alpha, N}^{r}$. Then for some $\varepsilon>0$, the set of $\hat{q}$ in $H_{\alpha, N}^{r} \cap\left\{\left\|\hat{q}-\hat{q}_{0}\right\|_{\alpha, N}<\varepsilon\right\}$ such that $I+A(\hat{q}, 0)$ has a kernel of dimension $m$ is contained in a smooth surface of codimension $m$ in $H_{\alpha, N}^{r}$. For all $\hat{q}$ on this surface $(I+A(\hat{q}, 0))$ has a kernel of dimension $\geqq 1$.

Proof. Let $P(\hat{q})=1 / 2 \pi i \int_{|\omega+1|=c}(A(\hat{q}, 0)-\omega I)^{-1} d \omega$, as in (5.1). Here $P$ has an $m$-dimensional range for $c$ sufficiently small, and is differentiable for $\left\|\hat{q}-\hat{q}_{0}\right\|_{\alpha, N}<c_{1}$ for $c_{1}$ sufficiently small. Since $A(\hat{q}, 0)$ leaves $H_{\alpha, N}^{r}$ invariant when $\hat{q} \in H_{\alpha, N}^{r}$, as one sees taking inverse Fourier transforms, $P(\hat{q})$ inherits this property. By construction $I+A(\hat{q}, 0)$ has an $m$-dimensional null space if and only if $(I+A(\hat{q}, 0)) P(\hat{q})=0$. Let $\hat{f}_{1}, \ldots, \hat{f}_{m}$ be a basis for range $P\left(\hat{q}_{0}\right)$. Note that, since $\hat{q}_{0} \in H_{\alpha, N}^{r}$, we may choose $\hat{f}_{i} \in H_{\alpha, N}^{r}$. Let

$$
\begin{aligned}
d_{i}(\hat{q}) & =(2 \pi)^{-3} \int_{\mathbf{R}^{3}} \frac{\overline{\hat{f}}_{1}(\xi)}{|\xi|^{2}}\left[(I+A(\hat{q}, 0)) P(\hat{q}) \hat{f}_{i}\right](\xi) d \xi \\
& =\int_{\mathbf{R}^{3}}\left[E_{0} f_{1}\right](x)\left[\left(I+q E_{0}\right) g_{i}\right](x) d x
\end{aligned}
$$

by Plancherel's theorem, where $g_{i}$ is the inverse Fourier transform of $P(\hat{q}) \hat{f}_{i}$, see Remark 3 after Theorem 3.1. The set of $\hat{q}$ for which $I+A(\hat{q}, 0)$ has an $m$-dimensional nullspace intersected with $\left\|\hat{q}-\hat{q}_{0}\right\|_{\alpha, N}<c_{1}$ is contained in

$$
\sum=\left\{\hat{q}: d_{i}(\hat{q})=0, i=1, \ldots, m\right\}
$$

and $d_{i}$ is real-valued on $H_{\alpha, N}^{r}$. Taking Frechet derivatives at $\hat{q}=\hat{q}_{0}$,

$$
d_{i}^{\prime}\left(\hat{q}_{0}\right) \hat{r} \equiv \int_{\mathbf{R}^{3}}\left(E_{0} f_{1}\right)\left(E_{0} f_{i}\right) r d x
$$

since $g_{i}\left(\hat{q}_{0}\right)=f_{i}$. Since $-\Delta E_{0} f_{i}+q_{0} E_{0} f_{i}=0$, unique continuation implies no $E_{0} f_{i}$ can vanish on an open set. The linear independence of $\left\{f_{i}\right\}_{i=1}^{m}$ implies the linear independence of $\left\{E_{0} f_{i}\right\}_{i=1}^{m}$. Thus we conclude $\left\{\left(E_{0} f_{1}\right)\left(E_{0} f_{i}\right)\right\}_{i=1}^{m}$ is linearly independent as well. Thus we may choose real-valued $\varphi_{j} \in C_{0}^{\infty}\left(\mathbf{R}^{3}\right), j=1, \ldots, m$ such that

$$
\int_{\mathbf{R}^{3}}\left(E_{0} f_{1}\right)\left(E_{0} f_{i}\right) \varphi_{j} d x= \begin{cases}1 & i=j \\ 0 & i \neq j\end{cases}
$$

Now we restrict $d_{1}, \ldots, d_{m}$ to $H_{\alpha, N}^{r}$ and let $H^{\prime}$ be a closed complementary subspace to span $\left\{\varphi_{j}\right\}_{j=1}^{m}$ in $H_{\alpha, N}^{r}$. By the implicit function theorem the system of equations

$$
d_{i}\left(\hat{q}^{\prime}+\sum_{j=1}^{m} s_{j} \hat{\varphi}_{j}\right)=0 \quad i=i, \ldots, m
$$

where $\hat{q} \in H^{\prime}$ and $s=\left(s_{1}, \ldots, s_{m}\right) \in \mathbf{R}^{m}$ can be solved for $s\left(\hat{q}^{\prime}\right)$ when $\hat{q}^{\prime}+\sum_{j=1}^{m} s_{j} \hat{\varphi}_{j}$ is near $\hat{q}_{0}$. Now we are ready to prove, 
Proposition 5.3. The set $\mathcal{O} \cap H_{\alpha, N}^{r}$ is contained in a connected component of $\mathcal{O}$.

Proof. Since $\mathcal{O} \cap H_{\alpha, N}^{r}$ is an open dense set in $H_{\alpha, N}^{r}$ by Theorem 3.1 and the density of $C_{0}^{\infty}$ in $H_{\alpha, N}$ implies the density of Fourier transforms of real-valued $C_{0}^{\infty}$ in $H_{\alpha, N}^{r}$, it will suffice to show that we can connect any pair of functions $\hat{q}_{1}$ and $\hat{q}_{2}$ in $\mathcal{O}$, when $q_{1}$ and $q_{2}$ are real-valued functions in $C_{0}^{\infty}\left(\mathbf{R}^{3}\right)$. Given two such functions, let $q(t)=t q_{1}+(1-t) q_{2}, t \in[0,1]$. Since $A(\hat{q}(t), 0)$ is real-analytic as an operator-valued function of $t$ on $H_{\alpha, N}$, and $I+A(\hat{q}(0), 0)$ is injective, $I+A(\hat{q}(t), 0)$ has a nontrivial nullspace for at most a finite set $S$ of $t$ in $[0,1]$.

Suppose that $t_{0} \in S$ and

$$
\operatorname{dim} \operatorname{Null}\left(I+A\left(\hat{q}\left(t_{0}\right), 0\right)\right)=m
$$

is maximal for $t \in[0,1]$. If $m>1$, we choose $\varepsilon_{0}$ small enough that $\operatorname{dim} \operatorname{Null}(I+A(\hat{q}(t), 0))<m$ for $0<\left|t-t_{0}\right|<\varepsilon_{0}$. Taking $\varepsilon_{0}$ smaller if necessary, we may assume $\left\|\hat{q}(t)-\hat{q}\left(t_{0}\right)\right\|_{\alpha, N}<\varepsilon_{1}$, where by Lemma 5.2 the set of $\hat{q}$ in $\left\|\hat{q}-\hat{q}\left(t_{0}\right)\right\|_{\alpha, N}<$ $\varepsilon_{1}$ such that $\operatorname{dim} \operatorname{Null}(I+A(\hat{q}, 0))=m$ is contained in the set of $\hat{q}^{\prime}+\sum_{j=1}^{m} s_{j}\left(\hat{q}^{\prime}\right) \hat{\varphi}_{j}$ with $\hat{q}^{\prime} \in H^{\prime}$, a closed complement of $\operatorname{span}\left\{\hat{\varphi}_{j}\right\}_{j=1}^{m}$ in $H_{\alpha, N}^{r}$. Then, $\hat{q}(t)=\hat{q}^{\prime}(t)+\sum_{j=1}^{m} r_{j}(t) \hat{\varphi}_{j}$ for $\left|t-t_{0}\right|<\varepsilon_{0}$, where $\hat{q}^{\prime}(t)$ and $r(t)$ are affine linear in $t$. Since $m>1$, the set in $\mathbf{R}^{m+1}$,

$$
\Sigma_{\delta, \varepsilon}=\left\{(u, s) \in \mathbf{R}^{m+1}:\left|s-s\left(\hat{q}^{\prime}\left(t_{0}\right)\right)\right|<\delta,\left|u-t_{0}\right| \leqq \varepsilon, s \neq s\left(\hat{q}^{\prime}(u)\right)\right\}
$$

is connected for all $\delta$ and $\varepsilon$. For $\delta$ sufficiently small

$$
\left\{\hat{q}^{\prime}+\sum_{j=1}^{m} s_{j} \hat{\varphi}_{j}:\left|s-s\left(\hat{q}^{\prime}\left(t_{0}\right)\right)\right|<\delta \text { and }\left\|\hat{q}^{\prime}-\hat{q}^{\prime}\left(t_{0}\right)\right\|_{\alpha, N}<\varepsilon_{1} / 2\right\}
$$

is contained in $\left\|\hat{q}-\hat{q}\left(t_{0}\right)\right\|<\varepsilon_{1}$. Likewise for $\varepsilon$ sufficiently small $(t, r(t)) \in \sum_{\delta, \varepsilon}$ for $\left|t-t_{0}\right|=\varepsilon$. Hence, we can replace $(t, r(t))$ by a piecewise linear function $(a(t), \tilde{r}(t))$ for $\left|t-t_{0}\right| \leqq \varepsilon$ such that $\tilde{r}\left(t_{0} \pm \varepsilon\right)=r\left(t_{0} \pm \varepsilon\right), a\left(t_{0} \pm \varepsilon\right)=t_{0} \pm \varepsilon$ and $(a(t), \tilde{r}(t)) \in \sum_{\delta, \varepsilon}$. Now we set

$$
\hat{q}_{1}(t)=\left\{\begin{array}{l}
\hat{q}(t) \text { for }\left|t-t_{0}\right|>\varepsilon \\
\hat{q}^{\prime}(a(t))+\sum_{j=1}^{m} \tilde{r}_{j}(t) \hat{\varphi}_{j} \text { for }\left|t-t_{0}\right| \leqq \varepsilon .
\end{array}\right.
$$

The function $\hat{q}_{1}(t)$ is piecewise linear, and, since $\mathcal{O} \cap H_{\alpha, N}^{r}$ is dense in $H_{\alpha, N}^{r}$, we may assume its corners are in $\mathcal{O}$.

Continuing in this way, we arrive at a piecewise linear function $\hat{q}_{N}(t)$ with corners in $\mathcal{O}$ such that $\operatorname{dim} \operatorname{Null}\left(I+A\left(\hat{q}_{N}(t), 0\right)\right)<m$ for $t \in[0,1], \hat{q}_{N}(0)=\hat{q}_{1}, \hat{q}_{N}(1)=\hat{q}_{2}$, and $q_{N}(t) \in C_{0}^{\infty}\left(\mathbf{R}^{3}\right)$ for $t \in[0,1]$. Since the set of $t$ in $[0,1]$ such that $\operatorname{dim} \operatorname{Null}(I+$ $\left.A\left(\hat{q}_{N}(t), 0\right)\right)>0$ is again finite, we can repeat the preceding argument until we have a piecewise linear $\hat{q}_{M}(t)$ with corners in $\mathcal{O}$ such that $\hat{q}_{M}(0)=\hat{q}_{1}, \hat{q}_{M}(1)=\hat{q}_{2}, q_{M}(t)$ is a real-valued function in $C_{0}^{\infty}\left(\mathbf{R}^{3}\right)$ for $t \in[0,1]$, and $\operatorname{dim} \operatorname{Null}\left(I+A\left(\hat{q}_{M}(t), 0\right)\right)<2$ for $t \in[0,1]$. Since $\left(I+A\left(\hat{q}_{M}(t), 0\right)\right)$ can have a nontrivial nullspace for only a finite number of $t$ in $[0,1]$, and $I+A\left(\hat{q}_{M}(t), k\right)$ does not have a nullspace for $k>0$, we complete the proof with a finite number of applications of Lemma 5.1. 
We are now ready to prove the main result of this work. Let $\mathcal{O}_{1}$ be the connected component of $\mathcal{O}$ containing $\mathcal{O} \cap H_{\alpha, N}^{r}$. Recall that $\mathcal{O} \cap H_{N}^{r}$ is dense in $H_{\alpha, N}^{r}$.

Theorem 5.4. The Frechet derivative of the backscattering map at $\hat{q}$ is an isomorphism of $H_{\alpha, N}$ when $\hat{q}$ belongs to an open, dense subset $\mathcal{O}_{2}$ of $\mathcal{O}_{1}$. Moreover, $\mathcal{O}_{2} \cap H_{\alpha, N}^{r}$ is dense in $H_{\alpha, N}^{r}$. By the implicit function theorem the backscattering map is an analytic homeomorphism on a neighborhood in $\mathrm{H}_{\alpha, N}$ of any $\hat{q} \in \mathrm{O}_{2}$.

Proof. The zero potential belongs to $\mathcal{O}_{1}$. Moreover, the Frechet derivative of the backscattering map at the zero potential is $[T f](\xi)=-f(2 \xi)$ which is an isomorphism. Thus, letting $\mathcal{O}_{2}$ be the subset of $\mathcal{O}_{1}$ for which $d S(\hat{q})$ is an isomorphism, $\mathcal{O}_{2}$ is nonempty. Since $d S(\hat{q})$ is analytic in $\hat{q}$ and Fredholm, $\mathcal{O}_{2}$ is open. If $\mathcal{O}_{2}$ is not dense in $\mathcal{O}_{1}$, then, since $\mathcal{O}_{1}$ is open and connected, the boundary of the interior of $\mathcal{O}_{2}^{c} \cap \mathcal{O}_{1}$ must be nonempty. Choose $\hat{q}_{0}$ in this set. Then any ball $B_{\varepsilon}=$ $\left\{\left\|\hat{q}-\hat{q}_{0}\right\|_{\alpha, N} \leqq \varepsilon\right\}$ must contain points in the interior of $\mathscr{O}_{2}^{c} \cap \mathcal{O}_{1}$ and in $\mathcal{O}_{2}$. Choose $\varepsilon$ small enough that $B_{\varepsilon} \subset \mathcal{O}_{1}$, and pick $\hat{q}_{1} \in \mathcal{O}_{2} \cap B_{\varepsilon}$ and $\hat{q}_{2} \in\left(\right.$ interior $\left.\mathcal{O}_{2}^{c} \cap \mathcal{O}_{1}\right) \cap B_{\varepsilon}$. Let

$$
\hat{q}(t)=t \hat{q}_{i}+(1-t) \hat{q}_{2} \quad t \in[0,1] .
$$

Since $d S(\hat{q})$ is analytic in $\hat{q}$ on $\mathcal{O}$ and Fredholm of index $0, d S(\hat{q}(t))$ can fail to be an isomorphism for only a finite number of $t$ in $[0,1]$. This contradicts $\hat{q}_{2} \in$ interior $\mathcal{O}_{2}^{c} \cap \mathcal{O}_{1}$, and hence $\mathcal{O}_{2}$ is dense in $\mathcal{O}_{1}$.

Now suppose that we have $\hat{q}_{0} \in H_{\alpha, N}^{r} \cap \mathcal{O}_{1}$ such that $d S(\hat{q})$ has a nontrivial kernel for $\hat{q} \in H_{\alpha, N}^{r}$ with $\left\|\hat{q}-\hat{q}_{0}\right\|_{\alpha, N}<\delta$, for some $\delta>0$. Introducing a finite rank operator $K$ such that $d S\left(\hat{q}_{0}\right)+K$ is invertible and taking the determinant of $(d S(\bar{q})+K)^{-1}(d S(\hat{q}))=I-(d S(\hat{q})+K)^{-1} K$, we get a $\mathbf{C}$-valued analytic function $\lambda(\hat{q})$ on $H_{\alpha, N}$ such that for $\left\|\hat{q}-\hat{q}_{0}\right\|_{\alpha, N}<\delta^{\prime}<\delta, d S(\hat{q})$ has a nontrivial kernel if and only if $\lambda(\hat{q})=0$. As the Fourier transform of a space of real-valued functions, $H_{\alpha, N}^{r}$ is a real subspace of $H_{\alpha, N}$, i.e. given $f \in H_{\alpha, N}, f=f_{1}+i f_{2}, f_{1}$ and $f_{2} \in H_{\alpha, N}^{r}$. It is a standard result that an analytic function vanishing on an open subset of a real subspace vanishes identically. One can see this by checking that complex Frechet derivatives of all orders must vanish on such a subset - as in the proof of this result for functions of one complex variable. Thus we conclude that $d S(\hat{q})$ has a nontrivial kernel for $\hat{q}$ in a neighborhood of $\hat{q}_{0}$ is $H_{\alpha, N}$. This contradicts the density of $\mathcal{O}_{2}$ in $\mathcal{O}_{1}$.

\section{Section 6. Real Potential and the Restricted Backscattering Map}

When we restrict the backscattering map to $H_{\alpha, N}^{r} \cap \mathcal{O}$, we cannot expect its range lie in $H_{\alpha, N}^{r}$. Since $H_{\alpha, N}^{r}$ is the Fourier transform of a space of real-valued functions, one natural way to proceed is to take the projection of backscattering which is the Fourier transform of taking the real part. Thus, we define the "restricted backscattering map":

$$
S_{r}: \hat{q} \rightarrow \frac{h(\xi,-\xi,|\xi|)+\bar{h}(-\xi, \xi,|\xi|)}{2} .
$$

Thus $S_{r}$ maps all of $H_{\alpha, N} \cap \mathcal{O}$ into $H_{\alpha, N}^{r}$. When we restrict $S_{r}$ to $H_{\alpha, N}^{r} \cap \mathcal{O}$, it is a 
real-analytic function with Frechet derivative given by (see (4.7)),

$$
\begin{aligned}
{\left[d S_{r}(\hat{q})\right](v)=} & -\frac{v(2 \xi)+\bar{v}(-2 \xi)}{2} \\
& -\pi^{-3} \int_{\mathbf{R}^{3}}\left[\frac{h(\xi-2 n,-\xi,|\xi|) v(2 n)}{|\xi-2 \eta|^{2}-(|\xi|+i 0)^{2}}+\frac{\bar{h}(-\xi-2 \eta, \xi,|\xi|) \bar{v}(2 \eta)}{|\xi+2 \eta|^{2}-(|\xi|-i 0)^{2}}\right] d \eta \\
& -2^{-4} \pi^{-6} \int_{\mathbf{R}^{3} \mathbf{R}^{3}}\left[\frac{h(\xi, \tau,|\xi|) h(\tau-2 \eta,-\xi,|\xi|) v(2 n)}{\left(|\tau|^{2}-(|\xi|+i 0)^{2}\right)\left(|2 \eta-\tau|^{2}-(|\xi|+i 0)^{2}\right)}\right. \\
& \left.+\frac{\bar{h}(-\xi, \tau,|\xi|) \bar{h}(\tau-2 \eta, \xi,|\xi|) \bar{v}(2 \eta)}{\left(|\tau|^{2}-(|\xi|-i 0)^{2}\right)\left(|2 \eta-\tau|^{2}-(|\xi|-i 0)\right)^{2}}\right] d \eta d t .
\end{aligned}
$$

If we make use of the identity $v(-\xi)=\bar{v}(\xi)$ and change variables in the appropriate integrals, this becomes

$$
\begin{aligned}
{\left[d S_{r}(\hat{q})\right](v)=} & -v(2 \xi) \\
& -\pi^{-3} \int_{\mathbf{R}^{3}}\left[\frac{h(\xi-2 \eta,-\xi,|\xi|)}{|\xi-2 \eta|^{2}-(|\xi|+i 0)^{2}}+\frac{\bar{h}(-\xi+2 \eta, \xi,|\xi|)}{|\xi-2 \eta|^{2}-(|\xi|-i 0)^{2}}\right] v(2 \eta) d \eta \\
& -2^{-4} \pi^{-6} \int_{\mathbf{R}^{3}} \int_{\mathbf{R}^{3}}\left[\frac{h(\xi, \tau,|\xi|) h(\tau-2 \eta,-\xi,|\xi|)}{\left(\mid \tau^{2}-(|\xi|+i 0)^{2}\right)\left(|2 \eta-\tau|^{2}-(|\xi|+i 0)^{2}\right)}\right. \\
& \left.+\frac{\bar{h}(-\xi, \tau,|\xi|) \bar{h}(\tau+2 \eta, \xi,|\xi|)}{\left(|\tau|^{2}-(|\xi|-i 0)^{2}\right)\left(|\tau+2 \eta|^{2}-(|\xi|-i 0)^{2}\right)}\right] v(2 \eta) d \eta d \tau .
\end{aligned}
$$

Thus $d S_{r}=(I+B+\underline{B}+C+\underline{C}) T$. The proof of Theorem 4.3 can be repeated without change to show that $B^{2}, \underline{B}^{2}, C$ and $\underline{C}$ are compact on $H_{\alpha, N}$. The proof applies to $B \underline{B}$ as well after one notes that changing $+i 0$ to $-i 0$ in one factor of the denominator of $T_{1}$ (see (4.9)) does not invalidate the proof: it merely interchanges the arguments for $S_{4}$ and $S_{5}$. Thus we conclude:

Theorem 6.1. $S_{r}$ is a real-analytic mapping of $H_{\alpha, N}^{r} \cap \mathcal{O}$ into $H_{\alpha, N}^{r}$ and its differential is a Fredholm operator of index zero.

Analogue of Theorem 5.4 here is the following theorem. Its proof coincides with the first paragraph of the proof of Theorem 5.4.

Theorem 6.2. Let $\mathcal{O}_{1}^{r}$ denote the component of $H_{\alpha, N}^{r} \cap \mathcal{O}$ containing the zero potential. Then the set $\mathcal{O}_{2}^{r}$ of $\hat{q} \in \mathcal{O}_{1}^{r}$ such that $d S_{r}(\hat{q})$ is an isomorphism of $H_{\alpha, N}^{r}$ is open and dense in $\mathcal{O}_{1}^{r}$. Hence, the implicit function theorem implies that $S_{r}$ is a real analytic homeomorphism on a neighborhood of each $\hat{q} \in \mathcal{O}_{2}^{r}$.

The set $\mathcal{O}_{1}^{r}$ is certainly not dense in $H_{\alpha, N}^{r}$. However, one does have the following.

Proposition 6.3. The set $\mathcal{O}_{1}^{r}$ contains all $\hat{q}$ such that $I+A(\hat{q}, 0)$ injective, $q \in C_{0}^{\infty}\left(\mathbf{R}^{3}\right)$ and $-\Delta+q$ has no negative eigenvalues as an operator on $L^{2}\left(\mathbf{R}^{3}\right)$.

Proof. It will suffice to show there is a curve $q(t)$ of real-valued functions in $C_{0}^{\infty}\left(\mathbf{R}^{3}\right)$ with $q(0)=q$ and $q\left(t_{0}\right)=0$ such that $I+A(\hat{q}(t), 0)$ is injective for $t \in\left[0, t_{0}\right]$.

Let $E_{0} f=(4 \pi)^{-1} \int_{\mathbf{R}^{3}}|x-y|^{-1} f(y) d y$. If $q$ is a real-valued function in $C_{0}^{\infty}\left(\mathbf{R}^{3}\right)$ 
and $-\Delta+q$ has no negative eigenvalues, we claim

$$
\int_{\mathbf{R}^{3}}\left(\overline{E_{0} f}\right)\left(f+q E_{0} f\right) d x \geqq 0
$$

for all $f \in C_{0}^{\infty}\left(\mathbf{R}^{3}\right)$. Let $\varphi$ be a smooth function satisfying $\varphi(x)=1$ for $|x|<1$ and $\varphi(x)=0$ for $|x|>2$. Let $\varphi_{R}(x)=\varphi(x / R)$. Given $f \in C_{0}^{\infty}\left(\mathbf{R}^{3}\right)$, let $u=E_{0} f$ and $u_{R}=\varphi_{R} u$. By assumption

$$
\int_{\mathbf{R}^{3}} \bar{u}_{R}\left(-\Delta u_{R}+q u_{R}\right) d x \geqq 0 .
$$

Since $|u|=0\left(|x|^{-1}\right)$ and $|\nabla u|=0\left(|x|^{-2}\right)$ for $|x|$ large, one checks easily that

$$
\lim _{R \rightarrow \infty} \int_{\mathbf{R}^{3}} \bar{u}_{R}\left(-\Delta u_{R}+q u_{R}\right) d x=\int_{\mathbf{R}^{3}} \bar{u}(-\Delta u+q u) d x,
$$

which implies (6.1).

If we now assume that $I+A(\hat{q}, 0)$ is injective on $H_{\alpha, N}$, it follows that $I+q E_{0}$ is injective on $C_{0}^{\infty}\left(\mathbf{R}^{3}\right)$. Since (6.1) implies that

$$
\left|\int_{\mathbf{R}^{3}}\left(\overline{E_{0} g}\right)\left(f+q E_{0} f\right) d x\right|^{2} \leqq \int_{\mathbf{R}^{3}}\left(\bar{E}_{0} g\right)\left(g+q E_{0} g\right) d x \int_{\mathbf{R}^{3}}\left(\overline{E_{0} f}\right)\left(f+q E_{0} f\right) d x
$$

for all $f, g \in C_{0}^{\infty}\left(\mathbf{R}^{3}\right)$, if $\int_{\mathbf{R}^{3}}\left(\overline{E_{0} f}\right)\left(f+q E_{0} f\right) d x=0$, then $\int_{\mathbf{R}^{3}}\left(\overline{E_{0} g}\right)\left(f+q E_{0} f\right) d x=0$ for all $g \in C_{0}^{\infty}\left(\mathbf{R}^{3}\right)$. Hence $f+q E_{0} f=0$, which contradicts the injectivity of $I+q E_{0}$, if $f \neq 0$. Thus

$$
\int_{\mathbf{R}^{3}}\left(\overline{E_{0} f}\right)\left(f+q E_{0} f\right) d x>0
$$

for all nonzero $f \in C_{0}^{\infty}\left(\mathbf{R}^{3}\right)$.

Let $\chi \in C_{0}^{\infty}\left(\mathbf{R}^{3}\right)$ be a nonnegative function which is identically 1 on the support of $q$. We define

$$
q(t)=\left\{\begin{array}{l}
q+t \chi, \quad t \in\left[0, t_{1}\right] \\
\left(t_{1}+1-t\right)\left(q+t_{1} \chi\right), \quad t \in\left[t_{1}, t_{1}+1\right]
\end{array}\right.
$$

where $t_{1}$ is chosen large enough that $q+t_{1} \chi$ is nonnegative. Now

$$
\int_{\mathbf{R}^{3}}\left(\overline{E_{0} f}\right)\left(f+q(t) E_{0} f\right) d x>0
$$

for all nonzero $f \in C_{0}^{\infty}\left(\mathbf{R}^{3}\right)$. For $t \in\left[0, t_{1}\right](6.3)$ follows from (6.2) and for $t \in\left[t_{1}, t_{1}+1\right]$ it follows from the strict positivity of $\int_{\mathbf{R}^{3}} f E_{0} \bar{f} d x$. If $I+A(\hat{q}(t), 0)$ had a null vector $\hat{f} \in H_{\alpha, N}$ for some $t \in\left[0, t_{1}+1\right]$, then one would have $\partial_{\xi}^{\beta} \hat{f} \in H_{\alpha, N^{\prime}}$ for all $N^{\prime}$ and $\beta$ by Lemma 3.3. Thus $f+q(t) E_{0} f=0$ and $E_{0} f \in C^{\infty}\left(\mathbf{R}^{3}\right)$. Hence $f \in C_{0}^{\infty}\left(\mathbf{R}^{3}\right)$ contradicting (6.3).

Acknowledgement. We wish to thank J.-C. Guillot for his critical reading of the manuscript of this article.

\section{References}

1. Agmon, S.: Spectral properties of Schroedinger operators and scattering theory. Ann. Scuola Norm. Sup. Pisa Cl. Sci. II, 2, 151-218 (1975) 
2. Beals, R., Coifman, R.: Multi-dimensional inverse scatterings and nonlinear partial differential equations. Proc. Symp. Pure Math. vol. 43, (Notre Dame, 1984), pp.45-70. Providence, RI: AMS 1985

3. Faddeev, L. D.: Mathematical aspects of the three body problem. Steklov Inst., Moscow 1963, vol. 69

4. Faddeev, L. D.: Inverse problem of quantum scattering theory, II. J. Sov. Math. 5, 334-396 (1976)

5. Friedrichs, K. O.: Perturbations of spectra in Hilbert spaces. Providence, RI: AMS 1965

6. Novikov, R. G., Khenkin, G. M.: The $\bar{\partial}$-equation in the multidimensional inverse scattering problem. Russ. Math. Surv., 42, 109-180 (1987)

7. Melin, A.: Some mathematical problems in inverse potential scattering. Sem. Equations aux Derivées Partielles 1986-87, Exp XX, Ecole Polytechnique

8. Bayliss, A., Li, Y., Morawetz, C.: Scattering by potential by hyperbolic methods. Math. Comput. (to appear)

9. Nachman, A., Ablowitz, M.: A multidimensional inverse scattering method. Stud. App. Math. 71, 243-250 (1984)

10. Nachbin, L.: Topology on spaces of holomorphic mappings. Ergebnisse der Mathematik und ihrer Grenzgebiete vol. 47. Berlin, Heidelberg, New York: Springer 1969

11. Newton, R.: Inverse scattering II, III, IV. J. Math. Phys. 21, 1698-1715 (1980); J. Math. Phys. 22, (1981); (Correction, J. Math. Phys. 23, 693 (1982); J. Math. Phys. 23, 594-604 (1982)

12. Pöschel, J., Trubowitz, E.: Inverse spectral theory. New York: Academic Press 1987

13. Prosser, R. T.: Formal solutions of inverse scattering problems I, II, III. J. Math. Phys. 10, 1819-1822 (1969); J. Math. Phys. 17, 1775-1779 (1976) and J. Math. Phys. 21, 2648-2653 (1980)

14. Stein, E.: Singular integrals and differentiability properties of functions. Princeton, NJ: Princeton University Press 1970

Communicated by B. Simon

Received July 29, 1988 
